\title{
Extension of the Chiral Perturbation Theory Meson Lagrangian to Order $p^{6}$
}

\author{
H. W. Fearing and S. Scherer* \\ TRIUMF, 4004 Wesbrook Mall, Vancouver, B. C., \\ Canada V6T 2A3
}

(August 22, 1994)

\begin{abstract}
We have derived the most general chirally invariant Lagrangian $\mathcal{L}_{6}$ for the meson sector at order $p^{6}$. The result provides an extension of the standard Gasser-Leutwyler Lagrangian $\mathcal{L}_{4}$ to one higher order, including as well all the odd intrinsic parity terms in the Lagrangian. The most difficult part of the derivation was developing a systematic strategy so as to get all of the independent terms and eliminate the redundant ones in an efficient way. The 'equation of motion' terms, which are redundant in the sense that they can be transformed away via field transformations, are separated out explicitly. The resulting Lagrangian has been separated into groupings of terms contributing to increasingly more complicated processes, so that one does not have to deal with the full result when calculating $p^{6}$ contributions to simple processes.
\end{abstract}

11.30.Rd, 12.39.Fe

*Address after Sept. 1, 1994: Institut für Kernphysik, Johannes Gutenberg-Universität, J. J. Becher-Weg 45, D-55099 Mainz, Germany 


\section{INTRODUCTION}

Ever since the early days of current algebra and the $P C A C$ hypothesis (for an overview see, e.g., Refs. [1] 3]), approximate chiral symmetry and its application in terms of effective Lagrangians [4 7] have been cornerstones of the description of the low-energy interactions of hadrons. In our present understanding, chiral symmetry is interpreted in terms of $Q C D$, the $S U(3)$ gauge theory of the strong interaction involving quarks and gluons (see, e.g., Refs. [8,9]). In the limit of massless $u, d$ and $s$ quarks, the $Q C D$ Hamiltonian exhibits a global $S U(3)_{L} \times S U(3)_{R}$ symmetry which is assumed to be spontaneously broken to $S U(3)_{V}$, giving rise to 8 massless Goldstone bosons. The experimentally observed small masses of the pseudoscalar octet $(\pi, K, \eta)$ then originate from an explicit symmetry breaking due to the finite quark masses (see Refs. [10,11] and references therein).

Extending a method originally proposed by Weinberg for the analysis of S-matrix elements [12], Gasser and Leutwyler [13,14] developed a technique which allows an expansion of $Q C D$ Green's functions of quark currents in terms of momenta and quark masses. Their procedure, now known as chiral perturbation theory (ChPT), makes use of an effective Lagrangian approach for the interaction between the Goldstone bosons $\pi, K, \eta$, and has been applied to a wide variety of processes, including interactions with external electromagnetic and electroweak probes (for pedagogical introductions and recent reviews, see Refs. [15 21]). The lowest-order Lagrangian corresponds to a nonlinear $\sigma$ model containing two free parameters, namely, the pion decay constant and the scalar quark condensate. At $O\left(p^{4}\right)$ the most general Lagrangian which is consistent with chiral symmetry, parity, and charge conjugation invariance contains 10 structures. The renormalized coefficients $L_{1} \ldots L_{10}$ have been empirically determined by fitting experimental data [13,14] (for a recent analysis see Ref. 222 and references therein). Theoretical predictions for the chiral coefficients have also been obtained by several authors using various techniques (see, e.g., Refs. [13,23 28]).

Without external fields (i.e., pure $Q C D$ ) or including electromagnetic processes only, the effective Lagrangian of Gasser and Leutwyler has an additional symmetry resulting in the property that it contains interaction terms involving exclusively an even number of Goldstone bosons [29,30]. Such interaction terms are sometimes also referred to as being of normal or even intrinsic parity. In Ref. [29] Witten discussed how to remove this symmetry which is not a symmetry of nature (for example, $\pi^{0} \rightarrow \gamma \gamma, K^{+} K^{-} \rightarrow \pi^{+} \pi^{-} \pi^{0}$, etc.). He essentially rederived the Wess-Zumino anomalous effective action describing the chiral anomaly [31]. The corresponding Lagrangian, which is of $O\left(p^{4}\right)$, cannot be written as a standard local effective Lagrangian in terms of the chiral matrix $U$ but can be expressed directly in terms of the boson fields. In particular, by construction it contains interaction terms with an odd number of Goldstone bosons (odd intrinsic parity). It was subsequently shown by several authors that quantum corrections to the Wess-Zumino classical action do not renormalize the coefficient of the $O\left(p^{4}\right)$ Wess-Zumino term 32 36]. Furthermore, the one-loop counter

terms lead to conventional chirally invariant structures at $O\left(p^{6}\right)$ [33 [36]. For a review of chiral perturbation theory involving the odd intrinsic parity sector we refer the reader to Ref. [30].

Chiral perturbation theory to $O\left(p^{4}\right)$ has become a well developed effective representation of $Q C D$ at low energies in terms of Goldstone bosons, with many applications. However, there exist cases where one-loop calculations to $O\left(p^{4}\right)$ do not appear to lead to satisfactory 
agreement with experimental data (see for example Refs. 37.38). As consistent two-loop calculations in the even intrinsic parity sector for particular processes have started to become available [39] it is clearly important and timely to extend the most general chiral effective Lagrangian to $O\left(p^{6}\right)$. Moreover, it is now also possible to calculate the $O\left(p^{6}\right)$ coefficients from phenomenological models [40].

The purpose of this work is to derive the most general structures of the chiral Lagrangian at $O\left(p^{6}\right)$ including the even, as well as the odd, intrinsic parity sector. The result provides an extension in analogous form of the standard Gasser-Leutwyler $O\left(p^{4}\right)$ Lagrangian to $O\left(p^{6}\right)$.

In the even intrinsic parity sector we find 111 independent terms. This part of the $O\left(p^{6}\right)$ Lagrangian will be of relevance for processes which vanish at $O\left(p^{2}\right)$, so that $O\left(p^{4}\right)$ is the leading order and the $O\left(p^{6}\right)$ terms provide the leading-order correction. A two-loop calculation of such a process, namely $\gamma \gamma \rightarrow \pi^{0} \pi^{0}$, has recently been reported in Ref. [39] (see also [41]). It is a feature of this process, and similarly of the decay $\eta \rightarrow \pi^{0} \gamma \gamma$ [37, that the $O\left(p^{4}\right)$ contribution is exclusively generated by one-loop diagrams. According to the power counting scheme [12], at $O\left(p^{6}\right)$ contributions result from two-loop diagrams with vertices from the $O\left(p^{2}\right)$ Lagrangian, one-loop diagrams with one vertex being $O\left(p^{2}\right)$ and the other $O\left(p^{4}\right)$ and, finally, from tree-level diagrams from the $O\left(p^{6}\right)$ effective Lagrangian.

In the odd intrinsic parity sector we find 32 independent structures of $O\left(p^{6}\right)$. Our result differs from previous determinations of these structures [34,36 which in turn are not in agreement with each other. We will provide a detailed comment on these differences. This sector has already been discussed in the literature in quite some detail [30], as it provides leading-order corrections to processes originating in the Wess-Zumino term.

At present it appears extremely unlikely that all renormalized coefficients at $O\left(p^{6}\right)$ may be determined empirically. However, there is the hope that for simple processes the much smaller subgroups of relevant coefficients can be determined by one experiment and then provide predictive power for a related process. Furthermore, theoretical techniques which resulted in predictions of the $O\left(p^{4}\right)$ coefficients might be extended to predict the coefficients at $O\left(p^{6}\right)$. For the corresponding discussion of techniques applying to the odd intrinsic parity sector see Ref. 30]. A derivation of the $O\left(p^{6}\right)$ even intrinsic parity sector using the NambuJona-Lasinio model will be provided in Ref. 40.

Our main emphasis in this paper will be on developing a systematic procedure for the derivation of the $O\left(p^{6}\right)$ Lagrangian so that the reader can be confident that the large number of terms we have found are indeed independent as well sufficient to describe the most general effective chiral Lagrangian at $O\left(p^{6}\right)$. The derivation involves various technical points, such as total-derivative arguments, trace-relations, use of the classical equation of motion in terms of field transformations, and special relations involving the completely antisymmetric tensor in four dimensions. We group the final structures according to the minimal number of Goldstone boson fields, assuming for the purpose of organizing the terms, a coupling to an external electromagnetic field. Our list will allow the reader to quickly identify the relevant terms at $O\left(p^{6}\right)$ needed for particular physical processes. The structures will, of course, be given in their full generality without any assumption concerning the nature of the external fields.

Our work is organized as follows. In Sec. II we discuss some of the general foundations of chiral perturbation theory, define the basic building blocks for the Lagrangian, discuss the strategy for obtaining a complete and independent set of terms, and describe a number 
of simplifications, symmetries and relations which will be used to reduce the result. In Sec. III we describe the details of the explicit construction of the Lagrangian. Sec. IV contains a discussion of the equation of motion terms, of some simplifications, and of a scheme of organization which groups the most useful terms together and a comparison with some previous works. The final results are given in Tables II - VII. Sec. $\nabla$ gives a brief summary. Details of the derivation of trace relations used in the simplifications, a table of the equation of motion terms, and some tables showing relations among terms which are not independent are relegated to the appendices.

The reader primarily interested in using the results should read Sec. II to understand the notation and the general strategy and Secs. $I \nabla$ and $\nabla$ for a listing and discussion of the results.

\section{CONSTRUCTION OF THE MOST GENERAL LAGRANGIAN $\mathcal{L}_{6}$ - INTRODUCTION AND BASIC APPROACH}

\section{A. Introduction}

Our aim in this section is to lay the groundwork for the construction of $\mathcal{L}_{6}$, the most general chirally invariant Lagrangian at order $p^{6}$. This Lagrangian will provide an extension to the next higher order of the standard Gasser-Leutwyler $\mathcal{L}_{4}$, as well as the odd intrinsic parity Lagrangian. Like $\mathcal{L}_{4}$ it will be expressed in terms of a set of basic building blocks, arranged in traces or products of traces such that the result is chirally invariant.

The main problem with such a construction is that it is far too easy to think of terms satisfying the necessary criteria. One quickly obtains many more terms than necessary, many that are not independent, though often not obviously so, and many expressed in a more complicated fashion than necessary.

Thus the most difficult task in such a construction is to develop a systematic strategy which allows one to get in an efficient manner all of the independent terms while at the same time eliminating, or preferably never generating, those terms which are redundant. Most of this section will be devoted to developing the various ingredients of this strategy. Then in the following major section the strategy will be applied to derive the most general $\mathcal{L}_{6}$. Some of this will be pedagogical, as it is important for the reader to be able to follow and understand the steps of the derivation and thus be convinced in the end that the result obtained is, in fact, complete and correct, something which is not necessarily possible when only a table of results is given.

To our knowledge there have been no previous attempts to generate the complete and most general $\mathcal{L}_{6}$, though there have been several attempts 34,36] to derive the set of odd intrinsic parity terms, i.e., those involving the completely antisymmetric tensor $\epsilon_{\alpha \beta \gamma \delta}$. In all of these attempts it appears that terms were missed, redundant terms were included, or both. 


\section{B. Chiral symmetry of the $Q C D$ Lagrangian}

Before discussing the construction of the effective chiral Lagrangian we will shortly review the chiral symmetry of the $Q C D$ Lagrangian coupled to external fields. This discussion is relevant for the identification of the transformation properties of the external sources under the group, parity, and charge conjugation.

Let us consider the $Q C D$ Lagrangian! [8,9],

$$
\mathcal{L}_{Q C D}=\sum_{f} \bar{q}_{f}\left(i \gamma^{\mu} D_{\mu}-m_{f}\right) q_{f}-\frac{1}{4} G_{\mu \nu}^{a} G_{a}^{\mu \nu} .
$$

In Eq. (1) $q_{f}$ denotes a quark field of flavor $f$ with current quark mass $m_{f}$. The covariant derivative is defined as

$$
D_{\mu} q_{f}=\left(\partial_{\mu}+i g \frac{\lambda^{a}}{2} A_{\mu}^{a}\right) q_{f}
$$

where, for simplicity, we do not exhibit the fact that the quark fields have three color indices. In Eq. (2) $g$ denotes the strong coupling constant, $\lambda^{a}$ are the usual Gell-Mann matrices, and $A_{\mu}^{a}$ represents a gluon field with color index $a$ which may take the values 1 to 8. Summation over repeated color indices is assumed. The gluon field strength tensor is defined as

$$
G_{\mu \nu}^{a}=\partial_{\mu} A_{\nu}^{a}-\partial_{\nu} A_{\mu}^{a}-g f_{a b c} A_{\mu}^{b} A_{\nu}^{c}
$$

where $f_{a b c}$ are the $S U(3)$ structure constants. We do not discuss the $\theta$-term which induces $P, T$ and $C P$ violations in the strong interactions [9].

In the following we will restrict ourselves to three flavors, $u, d, s$, and consider the limit $m_{u}, m_{d}, m_{s} \rightarrow 0$. In this limit Eq. (11) reduces to

$$
\mathcal{L}_{Q C D}^{0}=\sum_{f=u, d, s}\left(i \bar{q}_{L, f} \gamma^{\mu} D_{\mu} q_{L, f}+i \bar{q}_{R, f} \gamma^{\mu} D_{\mu} q_{R, f}\right)-\frac{1}{4} G_{\mu \nu}^{a} G_{a}^{\mu \nu},
$$

where the left-handed and right-handed quark fields are defined as

$$
q_{L}=\frac{1}{2}\left(1-\gamma_{5}\right) q, \quad q_{R}=\frac{1}{2}\left(1+\gamma_{5}\right) q
$$

The Lagrangian of Eq. (4) is invariant under the following global transformations of $q_{L}$ and $q_{R}$, respectively,

$$
\left(\begin{array}{c}
u_{L}^{\prime} \\
d_{L}^{\prime} \\
s_{L}^{\prime}
\end{array}\right)=U_{L}\left(\begin{array}{c}
u_{L} \\
d_{L} \\
s_{L}
\end{array}\right), \quad\left(\begin{array}{c}
u_{R}^{\prime} \\
d_{R}^{\prime} \\
s_{R}^{\prime}
\end{array}\right)=U_{R}\left(\begin{array}{c}
u_{R} \\
d_{R} \\
s_{R}
\end{array}\right)
$$

\footnotetext{
${ }^{1}$ We omit the gauge fixing term and the Faddeev-Popov ghost term, since they are not relevant for the discussion of chiral symmetry.
} 
where $U_{L}$ and $U_{R}$ are independent $U(3)$ matrices. This invariance, in principle, gives rise to 18 conserved currents. However, due to quantum effects, the axial $U(1)_{A}$ current is not conserved. In the following we will only be concerned with the analysis of the $G=$ $S U(3)_{L} \times S U(3)_{R}$ symmetry of Eq. (丰). It is generally accepted that this symmetry is spontaneously broken to $S U(3)_{V}$ giving rise to 8 Goldstone bosons. The finite quark masses in Eq. (1) then give rise to finite - but in comparison with other hadrons small - masses of the Goldstone bosons.

In order to systematically study the consequences of chiral symmetry and its breaking through the quark masses, we follow the technique of Gasser and Leutwyler [13,14] and introduce external c-number fields $v_{\mu}(x), a_{\mu}(x), s(x)$ and $p(x)$ into the Lagrangian,

$$
\mathcal{L}=\mathcal{L}_{Q C D}^{0}+\mathcal{L}_{\text {ext }}=\mathcal{L}_{Q C D}^{0}+\bar{q} \gamma^{\mu}\left(v_{\mu}+\gamma_{5} a_{\mu}\right) q-\bar{q}\left(s-i \gamma_{5} p\right) q
$$

The external fields are color neutral, hermitian $3 \times 3$ matrices, where the matrix character, with respect to the (suppressed) flavor indices $u, d, s$ of the quark fields, is

$$
v_{\mu}=\frac{\lambda^{a}}{2} v_{\mu}^{a}, \quad a_{\mu}=\frac{\lambda^{a}}{2} a_{\mu}^{a}, \quad s=\frac{\lambda^{a}}{2} s^{a}, \quad p=\frac{\lambda^{a}}{2} p^{a} .
$$

Of course, the three flavor $Q C D$ Lagrangian is recovered by setting $v_{\mu}=a_{\mu}=p=0$ and $s=\operatorname{diag}\left(m_{u}, m_{d}, m_{s}\right)$.

Requiring the total Lagrangian of Eq. (6) to be invariant under $P, C$ and $T$ leads to constraints on the transformation behavior of the external fields. Under a parity transformation the quark fields transform as

$$
q_{f}(\vec{x}, t) \stackrel{P}{\rightarrow} \gamma^{0} q_{f}(-\vec{x}, t)
$$

The requirement of invariance under a parity transformation,

$$
\mathcal{L}(\vec{x}, t) \stackrel{P}{\rightarrow} \mathcal{L}(-\vec{x}, t)
$$

results in the following transformation properties of the external fields

$$
v^{\mu} \stackrel{P}{\rightarrow} v_{\mu}, \quad a^{\mu} \stackrel{P}{\rightarrow}-a_{\mu}, \quad s \stackrel{P}{\rightarrow} s, \quad p \stackrel{P}{\rightarrow}-p
$$

where it is understood that the arguments change from $(\vec{x}, t)$ to $(-\vec{x}, t)$. Under charge conjugation the quark fields transform as

$$
q_{\alpha, f} \stackrel{C}{\rightarrow} C_{\alpha \beta} \bar{q}_{\beta, f}, \quad \bar{q}_{\alpha, f} \stackrel{C}{\rightarrow}-q_{\beta, f} C_{\beta \alpha}^{-1}
$$

In Eq. (11) the subscripts $\alpha$ and $\beta$ are Dirac spinor indices, $C=i \gamma^{2} \gamma^{0}$ is the usual charge conjugation matrix in the convention of Ref. [42] and $f$ refers to flavor. Using Eq. (11) it is straightforward to show that invariance of $\mathcal{L}_{\text {ext }}$ under charge conjugation requires the transformation properties

\footnotetext{
${ }^{2}$ We suppress color indices since we are considering color neutral external sources.
} 


$$
v_{\mu} \stackrel{C}{\rightarrow}-v_{\mu}^{T}, \quad a_{\mu} \stackrel{C}{\rightarrow} a_{\mu}^{T}, \quad s, p \stackrel{C}{\rightarrow} s^{T}, p^{T},
$$

where the transposition refers to the flavor space.

Finally, we discuss the properties of $\mathcal{L}$ under the group $G$. To that end we rewrite Eq. (6) in terms of $q_{L}$ and $q_{R}$,

$$
\mathcal{L}=\mathcal{L}_{Q C D}^{0}+\bar{q}_{L} \gamma^{\mu} L_{\mu} q_{L}+\bar{q}_{R} \gamma^{\mu} R_{\mu} q_{R}+\bar{q}_{L}(s-i p) q_{R}+\bar{q}_{R}(s+i p) q_{L}
$$

with $R_{\mu}=v_{\mu}+a_{\mu}$ and $L_{\mu}=v_{\mu}-a_{\mu}$. We then promote the global symmetry to a local symmetry. Eq. (13) remains invariant under $q_{R} \rightarrow V_{R}(x) q_{R}$ and $q_{L} \rightarrow V_{L}(x) q_{L}$, provided the external fields transform as

$$
\begin{aligned}
& R_{\mu} \stackrel{G}{\rightarrow} V_{R} R_{\mu} V_{R}^{\dagger}+i V_{R} \partial_{\mu} V_{R}^{\dagger}, \quad L_{\mu} \stackrel{G}{\rightarrow} V_{L} L_{\mu} V_{L}^{\dagger}+i V_{L} \partial_{\mu} V_{L}^{\dagger}, \\
& s-i p \stackrel{G}{\rightarrow} V_{L}(s-i p) V_{R}^{\dagger}, \quad s+i p \stackrel{G}{\rightarrow} V_{R}(s+i p) V_{L}^{\dagger} .
\end{aligned}
$$

\section{Basic Building Blocks}

The Lagrangian $\mathcal{L}_{6}$ is constructed from the same basic ingredients as $\mathcal{L}_{4}$, namely the Goldstone boson fields, external gauge fields, scalar and pseudoscalar external sources, and their derivatives. The 8 Goldstone bosons arising from the spontaneous symmetry breaking are collected in a $S U(3)$ matrix

$$
U(x)=\exp \left(i \frac{\phi(x)}{F_{0}}\right),
$$

with

$$
\phi(x)=\left(\begin{array}{ccc}
\pi^{0}+\frac{1}{\sqrt{3}} \eta & \sqrt{2} \pi^{+} & \sqrt{2} K^{+} \\
\sqrt{2} \pi^{-} & -\pi^{0}+\frac{1}{\sqrt{3}} \eta & \sqrt{2} K^{0} \\
\sqrt{2} K^{-} & \sqrt{2} \bar{K}^{0} & -\frac{2}{\sqrt{3}} \eta
\end{array}\right),
$$

and $F_{0}$ the pseudoscalar meson decay constant in the chiral limit [14. The matrix $U$ transforms linearly under the group $G=S U(3)_{L} \times S U(3)_{R}: U \rightarrow U^{\prime}=V_{R} U V_{L}^{\dagger}$ 14. Furthermore, under charge conjugation and parity the Goldstone bosons transform as $C$ : $\phi \rightarrow \phi^{T}$ and $P: \phi(\vec{x}, t) \rightarrow-\phi(-\vec{x}, t)$, or equivalently $C: U \rightarrow U^{T}$ and $P: U(\vec{x}, t) \rightarrow$ $U^{\dagger}(-\vec{x}, t)$.

We define the field strength tensors associated with the external gauge fields $R_{\mu}$ and $L_{\mu}$ as

$$
\begin{aligned}
F_{\mu \nu}^{R} & \equiv \partial_{\mu} R_{\nu}-\partial_{\nu} R_{\mu}-i\left[R_{\mu}, R_{\nu}\right] \\
F_{\mu \nu}^{L} & \equiv \partial_{\mu} L_{\nu}-\partial_{\nu} L_{\mu}-i\left[L_{\mu}, L_{\nu}\right]
\end{aligned}
$$

Note that $F_{\mu \nu}^{R}$ and $F_{\mu \nu}^{L}$ are hermitian, traceless, and gauge invariant, i.e., under the group they transform as $F_{\mu \nu}^{R} \stackrel{G}{\rightarrow} V_{R} F_{\mu \nu}^{R} V_{R}^{\dagger}$ and $F_{\mu \nu}^{L} \stackrel{G}{\rightarrow} V_{L} F_{\mu \nu}^{L} V_{L}^{\dagger}$. Furthermore, we follow Ref. 14 
and introduce the linear combination $\chi \equiv 2 B_{0}(s+i p)$, with $\chi \stackrel{G}{\rightarrow} V_{R} \chi V_{L}^{\dagger}$, where $B_{0}$ is related to the vacuum expectation value $<0|\bar{q} q| 0>$.

The effective Lagrangian is constructed in terms of $U, U^{\dagger}, \chi, \chi^{\dagger}$ and the field strength tensors $F_{\mu \nu}^{R}, F_{\mu \nu}^{L}$ as well as covariant derivatives of these objects. These covariant derivatives involve the gauge fields $R_{\mu}$ and $L_{\mu}$ and transform in the same way under the group as the quantities they act upon. Given the transformation properties of $R_{\mu}$ and $L_{\mu}$ of Eq. (14) we define the covariant derivatives as

$$
\begin{aligned}
A \stackrel{G}{\rightarrow} V_{R} A V_{L}^{\dagger}: & D_{\mu} A \equiv \partial_{\mu} A-i R_{\mu} A+i A L_{\mu}, \\
B \stackrel{G}{\rightarrow} V_{L} B V_{R}^{\dagger}: & D_{\mu} B \equiv \partial_{\mu} B+i B R_{\mu}-i L_{\mu} B, \\
C \stackrel{G}{\rightarrow} V_{R} C V_{R}^{\dagger}: & D_{\mu} C \equiv \partial_{\mu} C-i R_{\mu} C+i C R_{\mu}, \\
D \stackrel{G}{\rightarrow} V_{L} D V_{L}^{\dagger}: & D_{\mu} D \equiv \partial_{\mu} D-i L_{\mu} D+i D L_{\mu}, \\
E \stackrel{G \rightarrow E:}{\rightarrow} E & D_{\mu} E \equiv \partial_{\mu} E .
\end{aligned}
$$

Note that we use the same symbol $D_{\mu}$ for the covariant derivative, independent of the transformation property of the object it acts upon. The advantage of this convention is that a chain rule analogous to ordinary derivatives holds. Given the product $Z=X Y$ where $X, Y, Z$ have, according to Eq. (18), well-defined but not necessarily the same transformation behavior, the chain rule applies

$$
D_{\mu} Z=D_{\mu}(X Y)=\left(D_{\mu} X\right) Y+X\left(D_{\mu} Y\right)
$$

which is straightforward to verify using the definitions of Eq. (18).

This chain rule is valuable as an intermediate step in a number of the derivations of various relations. In essentially all cases however the final results will be expressed solely in terms of quantities transforming as $U$ and their covariant derivatives and thus require only the covariant derivative defined in the first line of the definitions of Eq. (18).

It will be very useful in the subsequent derivations to have all of the building blocks transform in the same way and also to be able to handle the external field terms $\chi, F_{\mu \nu}^{R}, F_{\mu \nu}^{L}$ in the same way. To that end we define

$$
\begin{aligned}
G^{\mu \nu} & \equiv F_{R}^{\mu \nu} U+U F_{L}^{\mu \nu}, \\
H^{\mu \nu} & \equiv F_{R}^{\mu \nu} U-U F_{L}^{\mu \nu} .
\end{aligned}
$$

We then let $\chi^{\mu \mu}=\chi$ and let $\chi^{\mu \nu}$, with $\mu \neq \nu$, stand for $G^{\mu \nu}$ or $H^{\mu \nu}$. Thus we can consider $\chi^{\mu \nu}$ with $\mu, \nu$ unspecified to stand for, collectively, the set $\left(\chi, G^{\mu \nu}, H^{\mu \nu}\right)$.

With these definitions, we have only two basic building blocks $U, \chi^{\mu \nu}$ and their adjoints and covariant derivatives acting on them. Note that by virtue of the chain rule, we do not need derivatives acting on products of these basic terms. All building blocks then transform as $U$ (or $U^{\dagger}$ ). In terms of the momentum expansion $U$ is of order $1, \chi^{\mu \nu}$ is of order $p^{2}$ and each covariant derivative $D_{\mu}$ is of order $p$ [14].

Finally, in order to construct terms which are invariant, we must put these basic pieces together so as to get quantities which transform as $V_{R} \ldots V_{R}^{\dagger}$. Thus define for any $A$ transforming as $V_{R} A V_{L}^{\dagger}$, i.e. as $U$, the hermitian (or antihermitian) combinations 


$$
[A]_{ \pm} \equiv \frac{1}{2}\left(A U^{\dagger} \pm U A^{\dagger}\right) .
$$

We can then take as the basic building blocks $[A]_{ \pm}$with $A$ taken as $\chi^{\mu \nu}$ or $D_{\mu} U$, or as some number of covariant derivatives acting on $\chi^{\mu \nu}$ or $D_{\mu} U$. The trace of a string of such quantities, or the product of such traces, will then be invariant under the group. Observe also that by defining the building blocks this way we include each operator and its adjoint. By using building blocks transforming as $V_{R} \ldots V_{R}^{\dagger}$ we also solve the problem of determining whether or not extra $U^{\prime}$ 's and $U^{\dagger}$ 's are necessary. In the usual approach, starting with a string of quantities transforming as $V_{R} \ldots V_{L}^{\dagger}$ or $V_{L} \ldots V_{R}^{\dagger}$, one can always insert extra $U$ 's and $U^{\dagger}$ 's in the string, replace some terms by their adjoints, and get another invariant string. One then must check in each case whether the resulting string is in fact independent of the original one. However by using the forms of Eq. (21) the only possible combination which can be inserted is $U U^{\dagger}$ which is unity and of course unnecessary. Observe also that if we were to insert $U U^{\dagger}$ between each term and after the last term in a trace of a string of terms of the form of Eq. (21) and use the relation $U^{\dagger}\left(A U^{\dagger} \pm U A^{\dagger}\right) U=U^{\dagger} A \pm A^{\dagger} U$ we obtain a trace of the same form as the original, but constructed of building blocks transforming as $V_{L} \ldots V_{L}^{\dagger}$ which we could have defined in a fashion analogous to Eq. (21). This shows that such terms are not independent and that terms of the form of Eq. (21) are sufficient.

To summarize, we have seen that the basic building blocks necessary to construct the most general chirally invariant Lagrangian are of the form $[A]_{ \pm}$, where $A$ is $D_{\mu} U$, $\chi^{\mu \nu}$ or (multiple) covariant derivatives of such quantities. The problem now, to be addressed in the next section, is how to put these building blocks together in an efficient way to get all possible terms in the Lagrangian.

\section{Strategy}

It is possible to generate a very large number of terms using just the building blocks defined in the previous section. The most difficult part of obtaining $\mathcal{L}_{6}$ is developing a strategy which obtains all of the independent terms without generating a lot of extraneous terms which have to be eliminated by hand.

To do this it is convenient to first define a hierarchy of terms. At each level we will then find the most general set of terms. First however, various relations can be used to eliminate some terms in favor of those lower down in the hierarchy. Since at each level we always find the most general set, it is not necessary to actually work out what these (often extremely complicated) relations are. To eliminate a term one must only show that there exists a relation expressing it in terms of quantities being kept at the same level and others lower down in the hierarchy.

We thus order the various classes of terms as follows:

I. Those terms with six $D_{\mu}$ 's.

II. Those terms with four $D_{\mu}$ 's and one $\chi^{\mu \nu}$.

III. Those terms with two $D_{\mu}$ 's and two $\chi^{\mu \nu}$ 's. 
IV. Those terms with three $\chi^{\mu \nu}$ s.

At each major level terms which involve a single trace are considered higher up than those with multiple traces. Likewise the ordering ensures that the addition of an $F^{\mu \nu}$ always results in a term lower in the hierarchy. Since the result must be a Lorentz scalar and since the only available tensors $\chi^{\mu \nu}$ and $\epsilon_{\alpha \beta \gamma \delta}$ have an even number of indices there are no terms with an odd number of $D_{\mu}$ 's.

For each level one first determines the form of the possible structures. For this it often is easier to start initially with the original building blocks $U, \chi^{\mu \nu}$ and their covariant derivatives. A number of simplifications and tricks to be discussed in the next section are then applied to reduce the number of possible terms and ensure that each term satisfies parity and charge conjugation. The remaining structures are then written in terms of the final building blocks $[A]_{ \pm}$. The result is a set of general forms. Explicit results can then be read off directly, usually by simply considering all possible permutations of the indices, and perhaps of the order of the terms, and all possible ways single traces can be broken up into multiple traces.

Terms involving $D_{\mu} D^{\mu} U$ are of particular interest as this factor appears in the classical equation of motion and terms proportional to the equation of motion can, as we shall see in a later section (see also Ref. [43]), be transformed away by a transformation on, or a redefinition of, the fields. Thus our strategy will be to extract in so far as it is possible such terms, which will be referred to as 'equation of motion terms'. At the end we can make such terms proportional to the full $\mathcal{L}_{2}$ equation of motion by simply adding in the rest of the terms in the equation of motion, as these terms will come from a lower level in the hierarchy.

\section{E. Simplifications-General Results}

\section{Total derivative arguments}

It is convenient to make use of the fact that a total derivative in the Lagrangian density does not change the equation of motion. This 'total derivative argument' can be applied as follows. For any pair of operators $A$ and $B$ which under the group either transform as $A^{\prime}=V_{R} A V_{L}^{\dagger}$ and $B^{\prime}=V_{L} B V_{R}^{\dagger}$ or as $A^{\prime}=V_{R} A V_{R}^{\dagger}$ and $B^{\prime}=V_{R} B V_{R}^{\dagger}$ the product $A B$ transforms as $V_{R} A B V_{R}^{\dagger}$ and the chain rule and definitions of the covariant derivatives yield

$$
D_{\mu}(A B)=\left(D_{\mu} A\right) B+A\left(D_{\mu} B\right)=\partial_{\mu}(A B)-i\left[R_{\mu}, A B\right]
$$

and thus using the fact that the trace of a commutator vanishes,

$$
\operatorname{Tr}\left(\left(D_{\mu} A\right) B\right)+\operatorname{Tr}\left(A\left(D_{\mu} B\right)\right)=\partial_{\mu} \operatorname{Tr}(A B) .
$$

The same result is obtained for combinations $A B$ transforming as $V_{L} A B V_{L}^{\dagger}$.

Clearly this result generalizes to products of terms of the form $B_{1} \ldots B_{n}$, and one gets

$$
\begin{aligned}
\operatorname{Tr}\left(\left(D_{\mu} B_{1}\right) B_{2} \ldots B_{n}\right) & +\operatorname{Tr}\left(B_{1}\left(D_{\mu} B_{2}\right) B_{3} \ldots B_{n}\right) \\
& +\ldots+\operatorname{Tr}\left(B_{1} \ldots\left(D_{\mu} B_{n}\right)\right)=\partial_{\mu}\left(\operatorname{Tr}\left(B_{1} \ldots B_{n}\right)\right) .
\end{aligned}
$$

It is also straightforward to extend the 'total derivative argument' to products of traces. As an illustration, we find applying Eqs. (23) and (19), 


$$
\begin{aligned}
\operatorname{Tr}\left(\left(D_{\mu} A_{1}\right) A_{2}\right) \operatorname{Tr}\left(A_{3} A_{4}\right) & +\operatorname{Tr}\left(A_{1}\left(D_{\mu} A_{2}\right)\right) \operatorname{Tr}\left(A_{3} A_{4}\right) \\
+\operatorname{Tr}\left(A_{1} A_{2}\right) \operatorname{Tr}\left(\left(D_{\mu} A_{3}\right) A_{4}\right) & +\operatorname{Tr}\left(A_{1} A_{2}\right) \operatorname{Tr}\left(A_{3}\left(D_{\mu} A_{4}\right)\right) \\
& =\partial_{\mu}\left(\operatorname{Tr}\left(A_{1} A_{2}\right) \operatorname{Tr}\left(A_{3} A_{4}\right)\right) .
\end{aligned}
$$

As a consequence of these arguments we may move covariant derivatives from one factor to another either within a single trace or across multiple traces in accord with Eqs. (23),(24),(25). Terms involving total derivatives will result, but these will not contribute to the equation of motion and thus can be dropped from the Lagrangian.

\section{Symmetrization of multiple covariant derivatives}

For any operator $A$ transforming as $U$ we have, using the definition of covariant derivatives Eq. (18) and of the field strength tensors Eq. (17)

$$
\left(D_{\mu} D_{\nu}-D_{\nu} D_{\mu}\right) A=i A F_{\mu \nu}^{L}-i F_{\mu \nu}^{R} A .
$$

Any term of the form $D_{\mu} D_{\nu} A$ can be written as a term symmetric and one antisymmetric in $\mu \leftrightarrow \nu$. Thus Eq. (26) implies that at any given level in the hierarchy $D_{\mu} D_{\nu} A$ can always be assumed to be symmetric in its indices, since the antisymmetric part can be expressed in terms of the field strength tensors and thus contributes only at a lower level in the hierarchy. Clearly this can be generalized to more than two covariant derivatives. Hence we will always assume that a multiple covariant derivative, $D_{\mu_{1}} \ldots D_{\mu_{n}} A$, is symmetric in the interchange of any of its indices. Formally this means that we will always interpret $D_{\mu} D_{\nu} A$ as

$$
D_{\mu} D_{\nu} A \rightarrow \frac{1}{2}\left(D_{\mu} D_{\nu}+D_{\nu} D_{\mu}\right) A,
$$

with an analogous definition when there are more than two covariant derivatives.

\section{Index exchange}

In keeping with the strategy of extracting as many 'equation of motion' factors as possible, one would like to arrange things so that covariant derivatives with summed indices, e.g. $D_{\alpha} D^{\alpha}$, act on the same factor $U$. For example consider $\operatorname{Tr}\left(\left(D_{\alpha} D_{\beta} A\right)\left(D^{\alpha} D_{\gamma} B\right) C\right)$, where $A, B, C$ do not contain any covariant derivatives. Using the results of Secs. [IE1 and [IE2 one can move the $D_{\beta}$ off of $A$ and on to $B$ and $C$ and then move the $D_{\alpha}$ off of $B$ and on to $A$ and $C$. The result is

$$
\begin{aligned}
\operatorname{Tr}\left(\left(D_{\alpha} D_{\beta} A\right)\left(D^{\alpha} D_{\gamma} B\right) C\right)= & \operatorname{Tr}\left(\left(D_{\alpha} D^{\alpha} A\right)\left(D_{\beta} D_{\gamma} B\right) C\right) \\
& + \text { terms with multiple } D \text { 's on only one of } A, B, C \\
& + \text { total derivatives, }
\end{aligned}
$$

where we have dropped the terms arising from the commutation of the $D$ 's at various stages which can be expressed via terms lower in the hierarchy using Eq. (26). 


\section{Building blocks involving multiple covariant derivatives}

Consider a term of the form $\left[D_{\alpha} D_{\beta} U\right]_{+}$. By starting with the trivial identity $0=$ $D_{\alpha} D_{\beta}\left(U U^{\dagger}\right)$ and using Eq. (19) one finds

$$
2\left[D_{\alpha} D_{\beta} U\right]_{+}=-\left(D_{\alpha} U\right)\left(D_{\beta} U\right)^{\dagger}-\left(D_{\beta} U\right)\left(D_{\alpha} U\right)^{\dagger}
$$

which means that $\left[D_{\alpha} D_{\beta} U\right]_{+}$can always be expressed in terms of quantities involving at most a single covariant derivative acting on $U$. This can be generalized, starting with $0=D_{\mu_{1}} \ldots D_{\mu_{n}}\left(U U^{\dagger}\right)$, to show that $\left[D_{\mu_{1}} \ldots D_{\mu_{n}} U\right]_{+}$can always be expressed as a sum of products of $[A]_{ \pm}$where A has at most one less covariant derivative. Symbolically, $\left[D^{n} U\right]_{+} \sim$ $\left(D^{i} U\right)\left(D^{n-i} U\right)^{\dagger} \sim\left[D^{i} U\right]_{ \pm}\left[D^{n-i} U\right]_{ \pm}$for all $(1 \leq i \leq n-1)$. By iteration, one then shows that all terms of the form $\left[D_{\mu_{1}} \ldots D_{\mu_{n}} U\right]_{+}$can be expressed via the combinations with relative negative sign $[A]_{-}$and the right (or left) hand side of Eq. (29). Note also that the simplest case $\left[D_{\mu} U\right]_{+}=0$, so that the right hand side of Eq. (29) can also be expressed solely in terms of $[A]_{-}$as well. This means in practice that we never need to include factors of the form $\left[D_{\mu_{1}} \ldots D_{\mu_{n}} U\right]_{+}$.

\section{Symmetries, parity and charge conjugation}

Each term in the final Lagrangian must be hermitian and must be invariant under parity and charge conjugation. Hermiticity is normally trivial as all terms come out to be either hermitian or antihermitian once parity and charge conjugation invariance is imposed. The strategy for ensuring $\mathrm{P}$ and $\mathrm{C}$ invariance is to take each candidate term and add to it the parity transform of the term, thus getting a result which is trivially $\mathrm{P}$ invariant. Similarly one then adds to this result its $\mathrm{C}$ transform, and so generates a $\mathrm{C}$ and $\mathrm{P}$ invariant term.

Consider first parity. We will be interested in candidate terms for the Lagrangian which are of the form of a trace of a string of factors of the canonical form $[A]_{ \pm}$, or alternatively a product of such traces, where $A$ can be $D_{\mu} U$ or $\chi^{\mu \nu}$ or their (perhaps multiple) covariant derivatives. Table $\mathbb{\text { I }}$ shows the transformation properties of the various factors. We see that under parity all allowed $A^{\prime}$ 's transform as $A \rightarrow(-1)^{p} A^{\dagger}$, where in addition all Lorentz indices in $A^{\dagger}$ have been raised (or lowered) with respect to those in $A$, and $\vec{x} \rightarrow-\vec{x}$. The quantity $p$ is an 'intrinsic parity' which is 1 for $H^{\mu \nu}$ and its covariant derivatives and 0 otherwise. In most cases Lorentz indices from one factor will be contracted with those of another factor, so that the raising or lowering of indices under $\mathrm{P}$ compensates in the two factors, and can be ignored. The exception to this is the case when the indices are contracted not with another term of the set of $A$ 's but with the antisymmetric tensor $\epsilon_{\alpha \beta \gamma \delta}$. Parity does not affect this tensor, so to get a Lorentz invariant quantity one must raise or lower its indices by hand. This introduces a minus sign since $\epsilon_{\alpha \beta \gamma \delta}=-\epsilon^{\alpha \beta \gamma \delta}$. This can be summarized neatly by including a $(-1)^{\epsilon}$ in the parity transform of a complete term, where $\epsilon$ counts the number of $\epsilon_{\alpha \beta \gamma \delta}$ 's in the term.

Using these preliminaries, we see that under parity $[A]_{ \pm} \rightarrow \pm(-1)^{p} U^{\dagger}[A]_{ \pm} U$. Under a trace the $U^{\dagger}$ and $U$ collapse to 1 , using the cyclic properties of a trace. Thus we arrive at the following operational method of making a candidate term in the Lagrangian invariant

under parity. Simply multiply the term by the factor $1+(-1)^{s+P+\epsilon}$ where $P$ is the sum of 
the intrinsic parities $p$ of the various terms, $\epsilon$ is the number of $\epsilon_{\alpha \beta \gamma \delta}$ 's in the term, and where the $(-1)^{s}$ is determined from the product of signs coming from the individual factors in the term, counting + for $[A]_{+}$and - for $[A]_{-}$. Clearly this holds for a single trace or a product of several traces.

Note also that by extracting this factor, we make it easy to see which terms are not allowed by parity conservation, namely those for which $(-1)^{s+P+\epsilon}$ is -1 .

Consider now charge conjugation and proceed in exactly the same way. From Table [1 it is easy to see that the necessary $A$ 's transform under $\mathrm{C}$ as $(-1)^{c} A^{T}$ where $c$ is an 'intrinsic charge conjugation quantum number' which is 1 for $G^{\mu \nu}$ and its covariant derivatives and 0 otherwise and $T$ is the transpose. We then have under charge conjugation $[A]_{ \pm} \rightarrow(-1)^{c}\left(U^{\dagger}[A]_{ \pm} U\right)^{T}$. Using the fact that the trace of a quantity is equal to the trace of its transpose, we then find that under $\mathrm{C}$ the trace of a string of basic factors $[A]_{ \pm}$goes simply to $(-1)^{C}$ times the trace with the basic factors in reverse order, where here $\mathrm{C}$ is simply the sum of the intrinsic charge conjugation quantum numbers of the various terms. Clearly in the case of multiple traces the factors are reversed in each trace individually.

Thus to summarize, the operational way to get a term in the Lagrangian to be invariant under charge conjugation is to add to it a term $(-1)^{C}$ times the term with the major factors reversed in each trace. If all of the traces have at most two factors, the cyclic property of a trace implies that the second term has the same structure as the first, and that effectively one is simply multiplying by the overall factor $\left(1+(-1)^{C}\right)$.

As noted ensuring hermiticity is relatively simple since the basic building blocks satisfy $[A]_{ \pm}^{\dagger}= \pm[A]_{ \pm}$. Thus the adjoint of a string of such terms is just $(-1)^{s}$ times the string in reversed order, where $s$ is calculated as in the parity discussion above. But, by virtue of $\mathrm{C}$ invariance, reversing the order produces an overall phase $(-1)^{C}$, so that taking the adjoint of a term simply generates an overall factor $(-1)^{s+C}=(-1)^{P+C+\epsilon}$. Thus to get a hermitian term we need only multiply by a factor of $i$ when this factor is -1 . This happens for terms with an even (odd) number of $G^{\mu \nu}$ 's and $H^{\mu \nu}$ 's (or their covariant derivatives) combined and one (no) $\epsilon_{\alpha \beta \gamma \delta}$ factor. We will do this only in the final listing of results.

\section{Trace relations}

In the construction of $\mathcal{L}_{4}$ a relation between $S U(3)$ generators was used to eliminate one term [14]. That relation can be generalized and used to eliminate a number of possible terms of order $p^{6}$. A proof of the relation and some discussion is given in Appendix A. For the present purposes we need only the result in the following form. For any set of $3 \times 3$ matrices $B_{i}, i=1, \ldots, n$ with $n \geq 4$, we can express

$$
\sum_{\text {All perm. }} \operatorname{Tr}\left(B_{1} \ldots B_{n}\right)
$$

as a sum of products of traces, each trace containing no more than $n-1$ of the $B_{i}$. Since we are interested only in terms invariant under the group, and since we take the $B_{i}$ in all permutations, we need $B_{i}$ to transform as $V_{R} B_{i} V_{R}^{\dagger}$. Thus the $B_{i}$ of interest will be the basic building blocks $[A]_{ \pm}$. Since a product of traces is always lower down in the hierarchy than a single trace, this relation allows us to discard one single trace term from each set of terms made up of all permutations of a particular group of building blocks. 


\section{Epsilon relations}

There is also a set of relations which relate various terms involving the tensor $\epsilon_{\alpha \beta \gamma \delta}$, and allow one to eliminate some in favor of others [36]. These relations originate from the observation that a tensor antisymmetric in five Lorentz indices must be zero since there are only four possible different indices. Such a tensor is

$$
g^{\alpha \beta} \epsilon^{\gamma \rho \tau \eta}-g^{\alpha \gamma} \epsilon^{\beta \rho \tau \eta}-g^{\alpha \rho} \epsilon^{\gamma \beta \tau \eta}-g^{\alpha \tau} \epsilon^{\gamma \rho \beta \eta}-g^{\alpha \eta} \epsilon^{\gamma \rho \tau \beta}=0 .
$$

Now consider a further tensor $Q_{\alpha \beta \gamma \rho \tau \eta}$ which will actually be the trace, or product of traces, of the basic building blocks $[A]_{ \pm}$and contract this tensor with that of Eq. (31) in all possible ways. This leads to the following six equations:

$$
\begin{aligned}
& \left(-Q_{\alpha}^{\alpha}{ }_{\gamma \rho \tau \eta}+Q_{\alpha \gamma}^{\alpha}{ }_{\rho \tau \eta}^{\alpha}-Q_{\alpha \gamma \rho}^{\alpha}{ }_{\tau \eta}^{\alpha}+Q_{\alpha \gamma \rho \tau}{ }_{\eta}^{\alpha}-Q_{\alpha \gamma \rho \tau \eta}{ }^{\alpha}\right) \epsilon^{\gamma \rho \tau \eta}=0, \\
& \left(+Q_{\alpha}^{\alpha}{ }_{\gamma \rho \tau \eta}^{\alpha} \quad+Q_{\gamma \alpha}^{\alpha}{ }_{\rho \tau \eta}^{\alpha}+Q_{\gamma \alpha \rho}{ }^{\alpha}{ }_{\tau \eta}-Q_{\gamma \alpha \rho \tau}{ }^{\alpha}{ }_{\eta}+Q_{\gamma \alpha \rho \tau \eta}{ }^{\alpha}\right) \epsilon^{\gamma \rho \tau \eta}=0, \\
& \left(-Q_{\alpha \gamma}{ }_{\rho \tau \eta}^{\alpha}+Q_{\gamma \alpha}{ }_{\rho \tau \eta}^{\alpha} \quad-Q_{\gamma \rho \alpha}{ }^{\alpha}{ }_{\tau \eta}+Q_{\gamma \rho \alpha \tau}{ }^{\alpha}{ }_{\eta}-Q_{\gamma \rho \alpha \tau \eta}{ }^{\alpha}\right) \epsilon^{\gamma \rho \tau \eta}=0, \\
& \left(+Q_{\alpha \gamma \rho}^{\alpha \rho_{\tau \eta}^{\alpha}}-Q_{\gamma \alpha \rho_{\tau \eta}}^{\alpha}+Q_{\gamma \rho_{\alpha}{ }_{\tau \eta}^{\alpha}} \quad-Q_{\gamma \rho \tau \alpha}{ }_{\eta}^{\alpha}+Q_{\gamma \rho \tau \alpha \eta}{ }^{\alpha}\right) \epsilon^{\gamma \rho \tau \eta}=0, \\
& \left(-Q_{\alpha \gamma \rho \tau}{ }^{\alpha}{ }_{\eta}+Q_{\gamma \alpha \rho \tau}{ }^{\alpha}{ }_{\eta}-Q_{\gamma \rho \alpha \tau}{ }_{\eta}^{\alpha}+Q_{\gamma \rho \tau \alpha}{ }^{\alpha}{ }_{\eta} \quad-Q_{\gamma \rho \tau \eta \alpha}{ }^{\alpha}\right) \epsilon^{\gamma \rho \tau \eta}=0, \\
& \left(+Q_{\alpha \gamma \rho \tau \eta}{ }^{\alpha}-Q_{\gamma \alpha \rho \tau \eta}{ }^{\alpha}+Q_{\gamma \rho \alpha \tau \eta}{ }^{\alpha}-Q_{\gamma \rho \tau \alpha \eta}{ }^{\alpha}+Q_{\gamma \rho \tau \eta \alpha}{ }^{\alpha} \quad\right) \epsilon^{\gamma \rho \tau \eta}=0 .
\end{aligned}
$$

Observe the symmetry of the $Q$ 's across the diagonal and the fact that Eq. (37) is just the sum of the first five. Thus in general there are five independent equations which generate for each specific $Q_{\alpha \beta \gamma \rho \tau \eta}$ five relations among the terms involving $\epsilon^{\gamma \rho \tau \eta}$. However if $Q_{\alpha \beta \gamma \rho \tau \eta}$ has some symmetry in its indices, not all of these five relations are independent. For example if $Q_{\alpha \beta \gamma \rho \tau \eta}$ is symmetric in the first two indices then Eqs. (34), (35), and (36) are identities and Eqs. (32) and (33) are not independent and so there is only one independent 'epsilon relation'.

\section{CONSTRUCTION OF THE MOST GENERAL LAGRANGIAN $\mathcal{L}_{6}$ - EXPLICIT CALCULATION}

Having completed these preliminaries we now proceed to the explicit calculation of $\mathcal{L}_{6}$. As noted earlier, the strategy will be to start with the highest level in our hierarchy and work down. At each level we first itemize the types of terms possible. At this stage it is useful to adopt a schematic notation. Thus an expression like $\left(D^{2} U\right)(D U)$ will mean the class of terms which have a $\left(D_{\mu} D_{\nu} U\right)$ or its adjoint and a $D_{\alpha} U$ or its adjoint, in any order, and with any contraction of indices which lead to a chirally invariant term when the trace is taken. We then use the results of Sec. $1 \mathrm{E}$ to eliminate as many classes of terms as possible 
and to force invariance under parity and charge conjugation. The results are a set of general expressions which satisfy the various constraints. One can then read directly from these expressions all of the allowed terms by simply taking all possible index contractions, and in some cases all possible orders.

At a given level in the hierarchy the manipulations required to reduce terms to lower levels and to ensure $\mathrm{P}$ and $\mathrm{C}$ invariance are essentially the same for multiple trace terms as for single trace ones. Hence we will generally work, somewhat symbolically, with just a string of factors and only at the end take the single trace and all possible multiple traces.

We will always attempt to extract a factor $\left[D_{\mu} D^{\mu} U\right]_{-}$as the terms containing this factor can be made proportional to the classical equation of motion, and as discussed below, transformed away via a transformation of the fields.

\section{A. Terms with six $D_{\mu}$ 's and no $\chi^{\mu \nu}$ 's}

Terms in this class will have six covariant derivatives acting singly or in multiples on $U$. Since there are six Lorentz indices, two must be contracted and the other four can be contracted pairwise in two pairs, or contracted with the indices of the antisymmetric tensor $\epsilon_{\alpha \beta \gamma \delta}$. Using the total derivative arguments of Sec. ПE 1 the covariant derivatives can always be moved back and forth, so as to get a factor $\left(D_{\mu} D_{\nu} U\right)$ which will be pulled to the first of the string of terms which eventually will become an argument of a trace. Either $\mu=\nu$ in this factor or, if not, no other multiple derivative has a contracted index in it, since if it did we could move all but the two contracted D's onto another term and pull the contracted pair out as the first term instead. Note that by virtue of Sec. ПIE2 all multiple derivatives can be taken as symmetric in their indices. This leads to structures of the following types:

$$
\begin{array}{ll}
\text { 1) } & \left(D_{\mu} D_{\nu} U\right)\left(D^{4} U\right), \\
\text { 2) } & \left(D_{\mu} D_{\nu} U\right)\left(D^{3} U\right)(D U) U, \\
\text { 3) } & \left(D_{\mu} D_{\nu} U\right)\left(D^{2} U\right)\left(D^{2} U\right) U, \\
\text { 4) } & \left(D_{\mu} D_{\nu} U\right)\left(D^{2} U\right)(D U)(D U), \\
\text { 5) } & \left(D_{\mu} D_{\nu} U\right)(D U)(D U)(D U)(D U) U .
\end{array}
$$

To get an expression transforming as $V_{R} \ldots V_{R}^{\dagger}$ so that the trace will be invariant we use Sec. IIE4 to write each of the structures in terms of products of factors $[A]_{ \pm}$. Each combination $[A]_{+}$, again by the results of Sec. [IE4, can be expressed via terms with one fewer covariant derivative, and hence via structures lower down in the list of Eq. (38), and so does not need to be kept. However at the bottom of the list $\left[D_{\mu} D_{\nu} U\right]_{+}$is expressed in terms of $(D U)(D U)$ and so generates a sixth structure involving six $[D U]_{-}$'s.

Requiring parity invariance introduces an overall factor $1+(-1)^{s+P+\epsilon}$ in accord with Sec. IIE5. The intrinsic parities $p$ are all zero so we find that the parity conserving terms must have an even number of $[A]_{-}$factors and no $\epsilon_{\alpha \beta \gamma \delta}$ or an odd number with an $\epsilon_{\alpha \beta \gamma \delta}$. Finally charge conjugation invariance simply adds in a term with the $[A]_{-}$factors in reverse order.

Thus we obtain terms of the following forms: 
1) $\left[D^{2} U\right]_{-}\left[D^{4} U\right]_{-}$,

2) $\left[D^{2} U\right]_{-}\left[D^{3} U\right]_{-}[D U]_{-} \epsilon_{\alpha \beta \gamma \delta}$,

3) $\left[D^{2} U\right]_{-}\left[D^{2} U\right]_{-}\left[D^{2} U\right]_{-} \epsilon_{\alpha \beta \gamma \delta}$,

4) $\left[D^{2} U\right]_{-}\left[D^{2} U\right]_{-}[D U]_{-}[D U]_{-}$,

5) $\quad\left[D^{2} U\right]_{-}[D U]_{-}[D U]_{-}[D U]_{-}[D U]_{-} \epsilon_{\alpha \beta \gamma \delta}$,

6) $[D U]_{-}[D U]_{-}[D U]_{-}[D U]_{-}[D U]_{-}[D U]_{-}$,

where in each case we must add a piece with all major factors in reverse order to ensure charge conjugation invariance. We must also take all possible starting orders, which in this case, once $\mathrm{C}$ is taken into account, is relevant only for 4) and generates two different structures there.

Note that all manipulations so far hold for single or multiple traces, except that for multiple traces we reverse the terms in each trace separately.

Finally we put in indices and the order reversal explicitly to obtain the most general result for single trace expressions involving six covariant derivatives. It consists of all possible index contractions of

$$
\begin{aligned}
1) & {\left[D_{\mu} D_{\nu} U\right]_{-}\left[D_{\alpha} D_{\beta} D_{\gamma} D_{\delta} U\right]_{-}, } \\
2) & {\left[D_{\mu} D_{\nu} U\right]_{-}\left(\left[D_{\alpha} D_{\beta} D_{\gamma} U\right]_{-}\left[D_{\delta} U\right]_{-}+\mathrm{rev}\right) \epsilon_{\rho \tau \eta \sigma}, } \\
3) & {\left[D_{\mu} D_{\nu} U\right]_{-}\left(\left[D_{\alpha} D_{\beta} U\right]_{-}\left[D_{\gamma} D_{\delta} U\right]_{-}+\mathrm{rev}\right) \epsilon_{\rho \tau \eta \sigma}, } \\
4 a) & {\left[D_{\mu} D_{\nu} U\right]_{-}\left(\left[D_{\alpha} D_{\beta} U\right]_{-}\left[D_{\gamma} U\right]_{-}\left[D_{\delta} U\right]_{-}+\mathrm{rev}\right), } \\
4 b) & {\left[D_{\mu} D_{\nu} U\right]_{-}\left(\left[D_{\alpha} U\right]_{-}\left[D_{\beta} D_{\gamma} U\right]_{-}\left[D_{\delta} U\right]_{-}+\mathrm{rev}\right), } \\
5) & {\left[D_{\mu} D_{\nu} U\right]_{-}\left(\left[D_{\alpha} U\right]_{-}\left[D_{\beta} U\right]_{-}\left[D_{\gamma} U\right]_{-}\left[D_{\delta} U\right]_{-}+\mathrm{rev}\right) \epsilon_{\rho \tau \eta \sigma}, } \\
6) & {\left[D_{\mu} U\right]_{-}\left[D_{\nu} U\right]_{-}\left[D_{\alpha} U\right]_{-}\left[D_{\beta} U\right]_{-}\left[D_{\gamma} U\right]_{-}\left[D_{\delta} U\right]_{-}+\text {rev. } }
\end{aligned}
$$

Here 'rev' stands for the terms in reverse order and it is understood that we must take all possible index contractions and in the end a trace.

The general result for multiple traces will be essentially the same, except that we need to take all possible multiple traces, as well as all index contractions, and except that the reversed term in each of the above is modified so as to reverse the terms in each trace separately.

We are now in a position to simply read off the results from Eq. (40), as the only thing that really needs to be done is to be sure that all independent index contractions are included. Note that a number of the possible contractions vanish because the multiple derivatives are symmetric in their indices while $\epsilon_{\alpha \beta \gamma \delta}$ is antisymmetric. Also we are interested only in the structure, so overall factors will be neglected.

For $\mu=\nu$ we get from 1)

$$
\operatorname{Tr}\left(\left[D_{\mu} D^{\mu} U\right]_{-}\left[D_{\alpha} D^{\alpha} D_{\beta} D^{\beta} U\right]_{-}\right),
$$

from $4 a)$

$$
\operatorname{Tr}\left(\left[D_{\mu} D^{\mu} U\right]_{-}\left[D_{\alpha} D^{\alpha} U\right]_{-}\left[D_{\beta} U\right]_{-}\left[D^{\beta} U\right]_{-}\right),
$$




$$
\operatorname{Tr}\left(\left[D_{\mu} D^{\mu} U\right]_{-}\left(\left[D_{\alpha} D_{\beta} U\right]_{-}\left[D^{\alpha} U\right]_{-}\left[D^{\beta} U\right]_{-}+\left[D^{\beta} U\right]_{-}\left[D^{\alpha} U\right]_{-}\left[D_{\alpha} D_{\beta} U\right]_{-}\right)\right),
$$

from $4 b)$

$$
\begin{aligned}
& \operatorname{Tr}\left(\left[D_{\mu} D^{\mu} U\right]_{-}\left[D_{\beta} U\right]_{-}\left[D_{\alpha} D^{\alpha} U\right]_{-}\left[D^{\beta} U\right]_{-}\right), \\
& \operatorname{Tr}\left(\left[D_{\mu} D^{\mu} U\right]_{-}\left[D^{\alpha} U\right]_{-}\left[D_{\alpha} D_{\beta} U\right]_{-}\left[D^{\beta} U\right]_{-}\right),
\end{aligned}
$$

and from 5)

$$
\operatorname{Tr}\left(\left[D_{\mu} D^{\mu} U\right]_{-}\left[D^{\alpha} U\right]_{-}\left[D^{\beta} U\right]_{-}\left[D^{\gamma} U\right]_{-}\left[D^{\delta} U\right]_{-}\right) \epsilon_{\alpha \beta \gamma \delta} .
$$

Terms from 2) or 3) all vanish, using the symmetry of multiple covariant derivatives.

For $\mu \neq \nu$, recall that we cannot have repeated indices in the multiple derivatives. Thus we obtain from $4 \mathrm{a}$ )

$$
\begin{aligned}
& \operatorname{Tr}\left(\left[D_{\mu} D_{\nu} U\right]_{-}\left[D^{\mu} D^{\nu} U\right]_{-}\left[D_{\alpha} U\right]_{-}\left[D^{\alpha} U\right]_{-}\right), \\
& \operatorname{Tr}\left(\left[D_{\mu} D_{\nu} U\right]_{-}\left[D^{\mu} D^{\alpha} U\right]_{-}\left[D^{\nu} U\right]_{-}\left[D_{\alpha} U\right]_{-}\right), \\
& \operatorname{Tr}\left(\left[D_{\mu} D_{\nu} U\right]_{-}\left[D^{\mu} D^{\alpha} U\right]_{-}\left[D_{\alpha} U\right]_{-}\left[D^{\nu} U\right]_{-}\right),
\end{aligned}
$$

from $4 b)$

$$
\begin{gathered}
\operatorname{Tr}\left(\left[D_{\mu} D_{\nu} U\right]_{-}\left[D_{\alpha} U\right]_{-}\left[D^{\mu} D^{\nu} U\right]_{-}\left[D^{\alpha} U\right]_{-}\right) \\
\operatorname{Tr}\left(\left[D_{\mu} D_{\nu} U\right]_{-}\left(\left[D^{\nu} U\right]_{-}\left[D^{\mu} D^{\alpha} U\right]_{-}\left[D_{\alpha} U\right]_{-}+\left[D_{\alpha} U\right]_{-}\left[D^{\mu} D^{\alpha} U\right]_{-}\left[D^{\nu} U\right]_{-}\right)\right)
\end{gathered}
$$

and from 5)

$$
\operatorname{Tr}\left(\left[D^{\mu} D^{\nu} U\right]_{-}\left(\left[D_{\nu} U\right]_{-}\left[D^{\beta} U\right]_{-}\left[D^{\gamma} U\right]_{-}\left[D^{\delta} U\right]_{-}+\left[D^{\delta} U\right]_{-}\left[D^{\gamma} U\right]_{-}\left[D^{\beta} U\right]_{-}\left[D_{\nu} U\right]_{-}\right)\right) \epsilon_{\mu \beta \gamma \delta},
$$

$$
\operatorname{Tr}\left(\left[D^{\mu} D^{\nu} U\right]_{-}\left(\left[D^{\beta} U\right]_{-}\left[D_{\nu} U\right]_{-}\left[D^{\gamma} U\right]_{-}\left[D^{\delta} U\right]_{-}+\left[D^{\delta} U\right]_{-}\left[D^{\gamma} U\right]_{-}\left[D_{\nu} U\right]_{-}\left[D^{\beta} U\right]_{-}\right)\right) \epsilon_{\mu \beta \gamma \delta}
$$

Finally from 6) we get

$$
\begin{aligned}
& \operatorname{Tr}\left(\left[D_{\alpha} U\right]_{-}\left[D^{\alpha} U\right]_{-}\left[D_{\beta} U\right]_{-}\left[D^{\beta} U\right]_{-}\left[D_{\gamma} U\right]_{-}\left[D^{\gamma} U\right]_{-}\right), \\
& \operatorname{Tr}\left(\left[D_{\alpha} U\right]_{-}\left[D^{\alpha} U\right]_{-}\left[D_{\beta} U\right]_{-}\left[D_{\gamma} U\right]_{-}\left[D^{\beta} U\right]_{-}\left[D^{\gamma} U\right]_{-}\right),
\end{aligned}
$$




$$
\begin{aligned}
& \operatorname{Tr}\left(\left[D_{\alpha} U\right]_{-}\left[D^{\alpha} U\right]_{-}\left[D_{\beta} U\right]_{-}\left[D_{\gamma} U\right]_{-}\left[D^{\gamma} U\right]_{-}\left[D^{\beta} U\right]_{-}\right), \\
& \operatorname{Tr}\left(\left[D_{\alpha} U\right]_{-}\left[D_{\beta} U\right]_{-}\left[D_{\gamma} U\right]_{-}\left[D^{\alpha} U\right]_{-}\left[D^{\beta} U\right]_{-}\left[D^{\gamma} U\right]_{-}\right), \\
& \operatorname{Tr}\left(\left[D_{\alpha} U\right]_{-}\left[D_{\beta} U\right]_{-}\left[D_{\gamma} U\right]_{-}\left[D^{\beta} U\right]_{-}\left[D^{\alpha} U\right]_{-}\left[D^{\gamma} U\right]_{-}\right) .
\end{aligned}
$$

A number of these terms differ from others only by a reordering of terms and thus we might expect that the trace relations of Sec. IIE6 might connect some of them. This is in fact the case. The sets Eqs. (42) and (44), Eqs. (43) and (45), Eqs. (47) and (50), Eqs. (48), (49), and (51), and Eqs. (54), (55), (56), (57), and (58) in each case contain all of the independent permutations. Hence one of each set can be eliminated using the trace relation, say Eqs. (44), (43), (50), (51), and (58). Likewise Eqs. (46), (52), and (53) are related by the epsilon relation of Sec. IIE7 and we can use it to eliminate say Eq. (53).

It is interesting to note that this epsilon relation connects a term involving the equation of motion, Eq. (46), with two that do not obviously contain the equation of motion, Eqs. (52) and (53). As we discuss later, the equation of motion terms can be transformed away by an appropriate transformation of the fields, and so do not contribute. Had we chosen to use the epsilon relation to eliminate the equation of motion term Eq. (46) rather than one of the others we would have ended up with two terms from this set instead of one, and it would have been extremely non obvious, from just looking at the terms, that one was in fact redundant.

Consider now the multiple trace terms arising from the general result of Eq. (40). We simply have to construct all possible combinations of traces, plus all possible contractions of indices. The terms arising from charge conjugation have to be modified slightly, as we take the terms in reverse order within each separate trace. The result is simplified very much by the fact that $\operatorname{Tr}\left(\left[D_{\mu} U\right]_{-}\right)=\operatorname{Tr}\left(\left[D_{\mu} D_{\nu} U\right]_{-}\right)=0$. That fact, plus the symmetry of multiple derivatives, means that there are no contributions from lines 1), 2), 3) or 5) of Eq. (40).

With $\mu=\nu$ we get from 4a)

$$
\begin{aligned}
& \operatorname{Tr}\left(\left[D_{\mu} D^{\mu} U\right]_{-}\left[D_{\alpha} D^{\alpha} U\right]_{-}\right) \operatorname{Tr}\left(\left[D_{\beta} U\right]_{-}\left[D^{\beta} U\right]_{-}\right), \\
& \operatorname{Tr}\left(\left[D_{\mu} D^{\mu} U\right]_{-}\left[D_{\alpha} D_{\beta} U\right]_{-}\right) \operatorname{Tr}\left(\left[D^{\alpha} U\right]_{-}\left[D^{\beta} U\right]_{-}\right),
\end{aligned}
$$

and from 4b)

$$
\begin{aligned}
& \operatorname{Tr}\left(\left[D_{\mu} D^{\mu} U\right]_{-}\left[D_{\alpha} U\right]_{-}\right) \operatorname{Tr}\left(\left[D_{\beta} D^{\beta} U\right]_{-}\left[D^{\alpha} U\right]_{-}\right) . \\
& \operatorname{Tr}\left(\left[D_{\mu} D^{\mu} U\right]_{-}\left[D_{\alpha} U\right]_{-}\right) \operatorname{Tr}\left(\left[D^{\alpha} D^{\beta} U\right]_{-}\left[D_{\beta} U\right]_{-}\right),
\end{aligned}
$$

With $\mu \neq \nu$ we get from $4 a$ )

$$
\operatorname{Tr}\left(\left[D_{\mu} D_{\nu} U\right]_{-}\left[D^{\mu} D^{\nu} U\right]_{-}\right) \operatorname{Tr}\left(\left[D_{\alpha} U\right]_{-}\left[D^{\alpha} U\right]_{-}\right),
$$




$$
\operatorname{Tr}\left(\left[D_{\mu} D_{\nu} U\right]_{-}\left[D^{\mu} D^{\alpha} U\right]_{-}\right) \operatorname{Tr}\left(\left[D^{\nu} U\right]_{-}\left[D_{\alpha} U\right]_{-}\right)
$$

and from $4 b)$

$$
\begin{aligned}
& \operatorname{Tr}\left(\left[D_{\mu} D_{\nu} U\right]_{-}\left[D_{\alpha} U\right]_{-}\right) \operatorname{Tr}\left(\left[D^{\mu} D^{\nu} U\right]_{-}\left[D^{\alpha} U\right]_{-}\right), \\
& \operatorname{Tr}\left(\left[D_{\mu} D_{\nu} U\right]_{-}\left[D^{\nu} U\right]_{-}\right) \operatorname{Tr}\left(\left[D^{\mu} D^{\alpha} U\right]_{-}\left[D_{\alpha} U\right]_{-}\right), \\
& \operatorname{Tr}\left(\left[D_{\mu} D_{\nu} U\right]_{-}\left[D_{\alpha} U\right]_{-}\right) \operatorname{Tr}\left(\left[D^{\mu} D^{\alpha} U\right]_{-}\left[D^{\nu} U\right]_{-}\right) .
\end{aligned}
$$

Finally from 6) we get

$$
\begin{aligned}
& \operatorname{Tr}\left(\left[D_{\mu} U\right]_{-}\left[D^{\mu} U\right]_{-}\right) \operatorname{Tr}\left(\left[D_{\alpha} U\right]_{-}\left[D^{\alpha} U\right]_{-}\left[D_{\beta} U\right]_{-}\left[D^{\beta} U\right]_{-}\right), \\
& \operatorname{Tr}\left(\left[D_{\mu} U\right]_{-}\left[D^{\mu} U\right]_{-}\right) \operatorname{Tr}\left(\left[D_{\alpha} U\right]_{-}\left[D_{\beta} U\right]_{-}\left[D^{\alpha} U\right]_{-}\left[D^{\beta} U\right]_{-}\right) \\
& \operatorname{Tr}\left(\left[D_{\mu} U\right]_{-}\left[D_{\nu} U\right]_{-}\right) \operatorname{Tr}\left(\left[D^{\mu} U\right]_{-}\left[D^{\nu} U\right]_{-}\left[D_{\alpha} U\right]_{-}\left[D^{\alpha} U\right]_{-}\right), \\
& \operatorname{Tr}\left(\left[D_{\mu} U\right]_{-}\left[D_{\nu} U\right]_{-}\right) \operatorname{Tr}\left(\left[D^{\mu} U\right]_{-}\left[D_{\alpha} U\right]_{-}\left[D^{\nu} U\right]_{-}\left[D^{\alpha} U\right]_{-}\right), \\
& \operatorname{Tr}\left(\left[D_{\mu} U\right]_{-}\left[D^{\mu} U\right]_{-}\left[D_{\alpha} U\right]_{-}\right) \operatorname{Tr}\left(\left[D_{\beta} U\right]_{-}\left[D^{\beta} U\right]_{-}\left[D^{\alpha} U\right]_{-}\right) \\
& \operatorname{Tr}\left(\left[D_{\mu} U\right]_{-}\left[D_{\nu} U\right]_{-}\left[D_{\alpha} U\right]_{-}\right) \operatorname{Tr}\left(\left[D^{\mu} U\right]_{-}\left[D^{\nu} U\right]_{-}\left[D^{\alpha} U\right]_{-}\right), \\
& \operatorname{Tr}\left(\left[D_{\mu} U\right]_{-}\left[D_{\nu} U\right]_{-}\left[D_{\alpha} U\right]_{-}\right) \operatorname{Tr}\left(\left[D^{\mu} U\right]_{-}\left[D^{\alpha} U\right]_{-}\left[D^{\nu} U\right]_{-}\right), \\
& \operatorname{Tr}\left(\left[D_{\mu} U\right]_{-}\left[D^{\mu} U\right]_{-}\right) \operatorname{Tr}\left(\left[D_{\alpha} U\right]_{-}\left[D^{\alpha} U\right]_{-}\right) \operatorname{Tr}\left(\left[D_{\beta} U\right]_{-}\left[D^{\beta} U\right]_{-}\right) \\
& \operatorname{Tr}\left(\left[D_{\mu} U\right]_{-}\left[D^{\mu} U\right]_{-}\right) \operatorname{Tr}\left(\left[D_{\alpha} U\right]_{-}\left[D_{\beta} U\right]_{-}\right) \operatorname{Tr}\left(\left[D^{\alpha} U\right]_{-}\left[D^{\beta} U\right]_{-}\right) \\
& \operatorname{Tr}\left(\left[D_{\mu} U\right]_{-}\left[D_{\nu} U\right]_{-}\right) \operatorname{Tr}\left(\left[D^{\mu} U\right]_{-}\left[D^{\alpha} U\right]_{-}\right) \operatorname{Tr}\left(\left[D^{\nu} U\right]_{-}\left[D_{\alpha} U\right]_{-}\right) .
\end{aligned}
$$

There are two trace relations for the multiple trace terms, one relating Eqs. (68) and (69) and one relating Eqs. (70) and (71). We use these to eliminate Eqs. (69) and (71). 


\section{B. Terms with four $D_{\mu}$ 's and one $\chi^{\mu \nu}$}

Terms at this level will have four $D_{\alpha}$ 's and one $\chi^{\mu \nu}$, which may be a simple $\chi$ if $\mu=\nu$ or may be $G^{\mu \nu}$ or $H^{\mu \nu}$ constructed from the $F^{\mu \nu}$ 's according to Eq. (20) if $\mu \neq \nu$. In the former case there will be four Lorentz indices which may be contracted in two pairs or with an $\epsilon_{\alpha \beta \gamma \delta}$ symbol. In the second case there will be six indices and the types of contractions will be the same as in the preceding section, except that $\chi^{\mu \nu}$ is antisymmetric in its indices.

Proceeding as before we write down schematically the types of terms which are possible, using the initial building blocks, with the understanding that eventually traces of these terms will be taken. We find

1) $\left(D^{2} U\right)\left(D^{2} \chi^{\mu \nu}\right)$

2) $\left(D^{2} U\right)\left(D^{2} U\right) \chi^{\mu \nu} U$,

3) $\left(D^{2} U\right)(D U)(D U) \chi^{\mu \nu}$,

4) $\left(D^{2} U\right)(D U)\left(D \chi^{\mu \nu}\right) U$

5) $\left(D^{2} \chi^{\mu \nu}\right)(D U)(D U) U$

6) $\left(D \chi^{\mu \nu}\right)(D U)(D U)(D U)$,

7) $\chi^{\mu \nu} U(D U)(D U)(D U)(D U)$.

In writing these down we have already used the total derivative arguments of Sec. [IE1 to eliminate terms involving $D^{4}$ and $D^{3}$. In fact it is a general result that one needs at most half of the covariant derivatives in the term acting on a single factor. We have included explicitly the minimum number of $U$ 's necessary to give an even number of factors so that it is possible to get the right overall transformation properties. Note also that at the present stage there may in principle be additional nonadjacent $U$ and $U^{\dagger}$ pairs distributed through some of the terms. Using again the total derivative argument one of the $D$ 's in 5) can be moved off of the $\chi$ to give terms like 6) and 4). Similarly in 6) the $D$ can be moved from the $\chi$ to give terms like 3 ) and, if there are extra $U, U^{\dagger}$ pairs, like 7). Thus 5) and 6) need not be considered.

Using Sec. IIE2 all multiple derivatives can be taken to be symmetric in their indices and this allows us to simplify 1) and 2). In these terms, to preserve Lorentz invariance, at least two D's must be contracted together, or contracted with an $\epsilon_{\alpha \beta \gamma \delta}$. Using the index exchange result of Sec. IIE. 3 these two $D$ 's can be brought together acting on a $U$, with the additional terms produced being either total derivatives or like some of the other terms. By symmetry the $\epsilon_{\alpha \beta \gamma \delta}$ term must vanish. Likewise by symmetry, the remaining two $D$ 's cannot be contracted with the $\chi^{\mu \nu}$, and so must also be contracted together. Thus $\chi^{\mu \nu}$ must be simply $\chi$ and we find for 1) and 2)

$$
\begin{aligned}
& 1) \rightarrow\left(D_{\alpha} D^{\alpha} U\right)\left(D_{\beta} D^{\beta} \chi\right), \\
& 2) \rightarrow\left(D_{\alpha} D^{\alpha} U\right)\left(D_{\beta} D^{\beta} U\right) \chi U .
\end{aligned}
$$

As before to get an expression transforming as $V_{R} \ldots V_{R}^{\dagger}$, so that the trace will be invariant, we write each factor in Eq. (78) in the form $[A]_{ \pm}$. Then Sec. IIE4 and particularly Eq. (29) allows us to eliminate $\left[D^{2} U\right]_{+}$in favor of terms we already have. 
The result of all these manipulations is the set of general forms

$$
\begin{array}{ll}
\text { 1) } & {\left[D_{\alpha} D^{\alpha} U\right]_{-}\left[D_{\beta} D^{\beta} \chi\right]_{ \pm}} \\
\text {2) } & {\left[D_{\alpha} D^{\alpha} U\right]_{-}\left[D_{\beta} D^{\beta} U\right]_{-}[\chi]_{ \pm}} \\
\text {3) } & {\left[D_{\alpha} D_{\beta} U\right]_{-}\left[D_{\gamma} U\right]_{-}\left[D_{\delta} U\right]_{-}\left[\chi^{\mu \nu}\right]_{ \pm}} \\
\text {4) } & {\left[D_{\alpha} D_{\beta} U\right]_{-}\left[D_{\gamma} U\right]_{-}\left[D_{\delta} \chi^{\mu \nu}\right]_{ \pm}} \\
7) & {\left[\chi^{\mu \nu}\right]_{ \pm}\left[D_{\alpha} U\right]_{-}\left[D_{\beta} U\right]_{-}\left[D_{\gamma} U\right]_{-}\left[D_{\delta} U\right]_{-}}
\end{array}
$$

where it is understood that we still must take all possible contractions of indices, perhaps with $\epsilon_{\alpha \beta \gamma \delta}$, and all different independent orderings of terms.

The last step is to impose parity and charge conjugation invariance. In accord with Sec. IIE5 requiring parity invariance simply leads to the overall factor $1+(-1)^{s+P+\epsilon}$ just as before. $\mathrm{P}$ is the sum of intrinsic parities $p$, but in effect here just counts the number of $H^{\mu \nu}$ 's, as all other quantities have $p=0$. For the five terms in Eq. (80) these factors are $(1 \mp 1),(1 \pm 1),\left(1 \mp(-1)^{P+\epsilon}\right),\left(1 \pm(-1)^{P+\epsilon}\right)$, and $\left(1 \pm(-1)^{P+\epsilon}\right)$, respectively, where the upper (lower) signs correspond just to the factors $[A]_{+}\left([A]_{-}\right)$involving $\chi$ or $\chi^{\mu \nu}$. Similarly to get charge conjugation invariance we must add a term $(-1)^{C} \times$ the original term with all the major factors $[A]_{ \pm}$in reverse order.

We can now put all of this together to get the general result for the four $D$ one $\chi^{\mu \nu}$ terms analogous to the six $D$ result of Eq. (40). It is most convenient to separate the $\mu=\nu$, i.e. $\chi$, terms from the $\mu \neq \nu$ terms as there are some 'accidental' simplifications which are different in the two cases, and since the values of the intrinsic parity and charge conjugation quantum numbers are different. For $\mu=\nu$ observe that the symmetry of the multiple derivatives ensures that there will be no $\epsilon_{\alpha \beta \gamma \delta}$ terms arising from 3) or 4) in Eq. (80). For $\mu \neq \nu$ we have by explicit calculation $\left[\chi^{\mu \nu}\right]_{-}=0$. Furthermore we can show, by taking the covariant derivative of $\left[\chi^{\mu \nu}\right]_{-}$and using the chain rule Eq. (19) that $\left[D_{\alpha} \chi^{\mu \nu}\right]_{-}$can be expressed in terms of other factors we have kept. Hence it is not independent and can be dropped.

We thus find the most general form for single trace expressions with four $D$ 's and one $\chi^{\mu \nu}$ to be, for $\mu=\nu$

$$
\begin{aligned}
\text { 1) } & {\left[D_{\alpha} D^{\alpha} U\right]_{-}\left[D_{\beta} D^{\beta} \chi\right]_{-}, } \\
\text {2) } & {\left[D_{\alpha} D^{\alpha} U\right]_{-}\left[D_{\beta} D^{\beta} U\right]_{-}[\chi]_{+}+\mathrm{rev}, } \\
\text { 3) } & {\left[D_{\alpha} D_{\beta} U\right]_{-}\left[D_{\gamma} U\right]_{-}\left[D_{\delta} U\right]_{-}[\chi]_{-}+\mathrm{rev}, } \\
\text { 4) } & {\left[D_{\alpha} D_{\beta} U\right]_{-}\left[D_{\gamma} U\right]_{-}\left[D_{\delta} \chi\right]_{+}+\mathrm{rev} } \\
7 a) & {[\chi]_{+}\left[D_{\alpha} U\right]_{-}\left[D_{\beta} U\right]_{-}\left[D_{\gamma} U\right]_{-}\left[D_{\delta} U\right]_{-}+\mathrm{rev}, } \\
7 b) & {[\chi]_{-}\left[D_{\alpha} U\right]_{-}\left[D_{\beta} U\right]_{-}\left[D_{\gamma} U\right]_{-}\left[D_{\delta} U\right]_{-} \epsilon^{\alpha \beta \gamma \delta}+\mathrm{rev} }
\end{aligned}
$$

and for $\mu \neq \nu$

$$
\begin{array}{ll}
3 a) & {\left[D_{\alpha} D_{\beta} U\right]_{-}\left[D_{\gamma} U\right]_{-}\left[D_{\delta} U\right]_{-}\left[G^{\mu \nu}\right]_{+} \epsilon_{\rho \tau \eta \sigma}-\mathrm{rev}} \\
3 b) & {\left[D_{\alpha} D_{\beta} U\right]_{-}\left[D_{\gamma} U\right]_{-}\left[D_{\delta} U\right]_{-}\left[H^{\mu \nu}\right]_{+}+\mathrm{rev},} \\
4 a) & {\left[D_{\alpha} D_{\beta} U\right]_{-}\left[D_{\gamma} U\right]_{-}\left[D_{\delta} G^{\mu \nu}\right]_{+}-\mathrm{rev}} \\
4 b) & {\left[D_{\alpha} D_{\beta} U\right]_{-}\left[D_{\gamma} U\right]_{-}\left[D_{\delta} H^{\mu \nu}\right]_{+} \epsilon_{\rho \tau \eta \sigma}+\mathrm{rev}}
\end{array}
$$




$$
\begin{array}{ll}
7 a) & {\left[G^{\mu \nu}\right]_{+}\left[D_{\alpha} U\right]_{-}\left[D_{\beta} U\right]_{-}\left[D_{\gamma} U\right]_{-}\left[D_{\delta} U\right]_{-}-\text {rev, }} \\
7 b) & {\left[H^{\mu \nu}\right]_{+}\left[D_{\alpha} U\right]_{-}\left[D_{\beta} U\right]_{-}\left[D_{\gamma} U\right]_{-}\left[D_{\delta} U\right]_{-} \epsilon_{\rho \tau \eta \sigma}+\text { rev. }}
\end{array}
$$

In both of these results 'rev' stands for a piece with the factors in reverse order and it is understood that we still must take all possible contractions of free indices and all possible independent orderings of factors and, to get the final contribution to the Lagrangian, take the trace.

Everything done to derive these results holds also essentially unchanged for multiple traces, by virtue of the total derivative arguments of Eqs. (24) and (25). We simply must take all possible combinations of traces, instead of a single trace, and must reverse the order of the terms in each individual trace separately.

It is now straightforward to tabulate the terms arising from Eqs. (81) and (82). Starting with Eq. (81) we get for the terms involving $\chi$ from 1)

$$
\operatorname{Tr}\left(\left[D_{\alpha} D^{\alpha} U\right]_{-}\left[D_{\beta} D^{\beta} \chi\right]_{-}\right),
$$

from 2)

$$
\operatorname{Tr}\left(\left[D_{\alpha} D^{\alpha} U\right]_{-}\left[D_{\beta} D^{\beta} U\right]_{-}[\chi]_{+}\right),
$$

from 3)

$$
\begin{gathered}
\operatorname{Tr}\left(\left[D_{\alpha} D^{\alpha} U\right]_{-}\left(\left[D_{\beta} U\right]_{-}\left[D^{\beta} U\right]_{-}[\chi]_{-}+[\chi]_{-}\left[D_{\beta} U\right]_{-}\left[D^{\beta} U\right]_{-}\right)\right), \\
\operatorname{Tr}\left(\left[D_{\alpha} D^{\alpha} U\right]_{-}\left[D_{\beta} U\right]_{-}[\chi]_{-}\left[D^{\beta} U\right]_{-}\right), \\
\operatorname{Tr}\left(\left[D_{\alpha} D_{\beta} U\right]_{-}\left(\left[D^{\alpha} U\right]_{-}\left[D^{\beta} U\right]_{-}[\chi]_{-}+[\chi]_{-}\left[D^{\beta} U\right]_{-}\left[D^{\alpha} U\right]_{-}\right)\right), \\
\operatorname{Tr}\left(\left[D_{\alpha} D_{\beta} U\right]_{-}\left[D^{\alpha} U\right]_{-}[\chi]_{-}\left[D^{\beta} U\right]_{-}\right),
\end{gathered}
$$

from 4)

$$
\begin{aligned}
& \operatorname{Tr}\left(\left[D_{\alpha} D^{\alpha} U\right]_{-}\left(\left[D_{\beta} U\right]_{-}\left[D^{\beta} \chi\right]_{+}+\left[D^{\beta} \chi\right]_{+}\left[D_{\beta} U\right]_{-}\right)\right), \\
& \operatorname{Tr}\left(\left[D_{\alpha} D_{\beta} U\right]_{-}\left(\left[D^{\alpha} U\right]_{-}\left[D^{\beta} \chi\right]_{+}+\left[D^{\beta} \chi\right]_{+}\left[D^{\alpha} U\right]_{-}\right)\right),
\end{aligned}
$$

and from 7)

$$
\begin{aligned}
& \operatorname{Tr}\left([\chi]_{+}\left[D_{\alpha} U\right]_{-}\left[D^{\alpha} U\right]_{-}\left[D_{\beta} U\right]_{-}\left[D^{\beta} U\right]_{-}\right), \\
& \operatorname{Tr}\left([\chi]_{+}\left[D_{\alpha} U\right]_{-}\left[D_{\beta} U\right]_{-}\left[D^{\alpha} U\right]_{-}\left[D^{\beta} U\right]_{-}\right),
\end{aligned}
$$




$$
\begin{gathered}
\operatorname{Tr}\left([\chi]_{+}\left[D_{\alpha} U\right]_{-}\left[D_{\beta} U\right]_{-}\left[D^{\beta} U\right]_{-}\left[D^{\alpha} U\right]_{-}\right), \\
\operatorname{Tr}\left([\chi]_{-}\left[D_{\alpha} U\right]_{-}\left[D_{\beta} U\right]_{-}\left[D_{\gamma} U\right]_{-}\left[D_{\delta} U\right]_{-}\right) \epsilon^{\alpha \beta \gamma \delta} .
\end{gathered}
$$

It is equally straightforward to read off the multiple trace terms originating from Eq. (81). Recall that $\operatorname{Tr}\left(\left[D_{\alpha} D_{\beta} U\right]_{-}\right)=\operatorname{Tr}\left(\left[D_{\alpha} U\right]_{-}\right)=0$. Thus we have no terms from 1$)$ and get from 2)

$$
\operatorname{Tr}\left(\left[D_{\alpha} D^{\alpha} U\right]_{-}\left[D_{\beta} D^{\beta} U\right]_{-}\right) \operatorname{Tr}\left([\chi]_{+}\right),
$$

from 3)

$$
\begin{aligned}
& \operatorname{Tr}\left(\left[D_{\alpha} D^{\alpha} U\right]_{-}\left[D_{\beta} U\right]_{-}\right) \operatorname{Tr}\left(\left[D^{\beta} U\right]_{-}[\chi]_{-}\right), \\
& \operatorname{Tr}\left(\left[D_{\alpha} D^{\alpha} U\right]_{-}[\chi]_{-}\right) \operatorname{Tr}\left(\left[D_{\beta} U\right]_{-}\left[D^{\beta} U\right]_{-}\right), \\
& \operatorname{Tr}\left(\left[D_{\alpha} D^{\alpha} U\right]_{-}\left[D_{\beta} U\right]_{-}\left[D^{\beta} U\right]_{-}\right) \operatorname{Tr}\left([\chi]_{-}\right), \\
& \operatorname{Tr}\left(\left[D_{\alpha} D_{\beta} U\right]_{-}\left[D^{\alpha} U\right]_{-}\right) \operatorname{Tr}\left(\left[D^{\beta} U\right]_{-}[\chi]_{-}\right), \\
& \operatorname{Tr}\left(\left[D_{\alpha} D_{\beta} U\right]_{-}[\chi]_{-}\right) \operatorname{Tr}\left(\left[D^{\alpha} U\right]_{-}\left[D^{\beta} U\right]_{-}\right), \\
& \operatorname{Tr}\left(\left[D_{\alpha} D_{\beta} U\right]_{-}\left[D^{\alpha} U\right]_{-}\left[D^{\beta} U\right]_{-}\right) \operatorname{Tr}\left([\chi]_{-}\right),
\end{aligned}
$$

from 4)

$$
\begin{aligned}
& \operatorname{Tr}\left(\left[D_{\alpha} D^{\alpha} U\right]_{-}\left[D_{\beta} U\right]_{-}\right) \operatorname{Tr}\left(\left[D^{\beta} \chi\right]_{+}\right), \\
& \operatorname{Tr}\left(\left[D_{\alpha} D_{\beta} U\right]_{-}\left[D^{\alpha} U\right]_{-}\right) \operatorname{Tr}\left(\left[D^{\beta} \chi\right]_{+}\right),
\end{aligned}
$$

and from 7)

$$
\begin{aligned}
& \operatorname{Tr}\left([\chi]_{+}\right) \operatorname{Tr}\left(\left[D_{\alpha} U\right]_{-}\left[D^{\alpha} U\right]_{-}\left[D_{\beta} U\right]_{-}\left[D^{\beta} U\right]_{-}\right), \\
& \operatorname{Tr}\left([\chi]_{+}\right) \operatorname{Tr}\left(\left[D_{\alpha} U\right]_{-}\left[D_{\beta} U\right]_{-}\left[D^{\alpha} U\right]_{-}\left[D^{\beta} U\right]_{-}\right), \\
& \operatorname{Tr}\left([\chi]_{+}\left[D_{\alpha} U\right]_{-}\right) \operatorname{Tr}\left(\left[D^{\alpha} U\right]_{-}\left[D_{\beta} U\right]_{-}\left[D^{\beta} U\right]_{-}\right),
\end{aligned}
$$




$$
\begin{gathered}
\operatorname{Tr}\left([\chi]_{+}\left[D_{\alpha} U\right]_{-}\left[D^{\alpha} U\right]_{-}\right) \operatorname{Tr}\left(\left[D_{\beta} U\right]_{-}\left[D^{\beta} U\right]_{-}\right), \\
\operatorname{Tr}\left([\chi]_{+}\left[D_{\alpha} U\right]_{-}\left[D_{\beta} U\right]_{-}\right) \operatorname{Tr}\left(\left[D^{\alpha} U\right]_{-}\left[D^{\beta} U\right]_{-}\right), \\
\operatorname{Tr}\left([\chi]_{+}\right) \operatorname{Tr}\left(\left[D_{\alpha} U\right]_{-}\left[D^{\alpha} U\right]_{-}\right) \operatorname{Tr}\left(\left[D_{\beta} U\right]_{-}\left[D^{\beta} U\right]_{-}\right), \\
\operatorname{Tr}\left([\chi]_{+}\right) \operatorname{Tr}\left(\left[D_{\alpha} U\right]_{-}\left[D_{\beta} U\right]_{-}\right) \operatorname{Tr}\left(\left[D^{\alpha} U\right]_{-}\left[D^{\beta} U\right]_{-}\right) .
\end{gathered}
$$

Like in the previous section there are here also several sets of terms consisting of all permutations of the factors. Thus we can use the trace relations of Sec. IIE6 to express one of each set in terms of the others and of terms with more traces which are lower down in the hierarchy. In particular Eqs. (85) and (86), Eqs. (87) and (88), Eqs. (91), (92), and (93) and Eqs. (104) and (105) form such sets and we choose to eliminate Eqs. (85), (87), (92), and (105) using the trace relations.

We now evaluate the terms coming from Eq. (82) which contain $G^{\mu \nu}$ and $H^{\mu \nu}$. We obtain for the single trace terms from $3 \mathrm{a}$ )

$$
\begin{gathered}
\operatorname{Tr}\left(\left[D_{\alpha} D^{\alpha} U\right]_{-}\left(\left[D^{\beta} U\right]_{-}\left[D^{\gamma} U\right]_{-}\left[G^{\mu \nu}\right]_{+}-\left[G^{\mu \nu}\right]_{+}\left[D^{\gamma} U\right]_{-}\left[D^{\beta} U\right]_{-}\right)\right) \epsilon_{\beta \gamma \mu \nu}, \\
\operatorname{Tr}\left(\left[D_{\alpha} D^{\alpha} U\right]_{-}\left[D^{\beta} U\right]_{-}\left[G^{\mu \nu}\right]_{+}\left[D^{\gamma} U\right]_{-}\right) \epsilon_{\beta \gamma \mu \nu}, \\
\operatorname{Tr}\left(\left[D^{\alpha} D^{\beta} U\right]_{-}\left(\left[D_{\beta} U\right]_{-}\left[D^{\gamma} U\right]_{-}\left[G^{\mu \nu}\right]_{+}-\left[G^{\mu \nu}\right]_{+}\left[D^{\gamma} U\right]_{-}\left[D_{\beta} U\right]_{-}\right)\right) \epsilon_{\alpha \gamma \mu \nu}, \\
\operatorname{Tr}\left(\left[D^{\alpha} D^{\beta} U\right]_{-}\left(\left[D_{\beta} U\right]_{-}\left[G^{\mu \nu}\right]_{+}\left[D^{\gamma} U\right]_{-}-\left[D^{\gamma} U\right]_{-}\left[G^{\mu \nu}\right]_{+}\left[D_{\beta} U\right]_{-}\right)\right) \epsilon_{\alpha \gamma \mu \nu}, \\
\operatorname{Tr}\left(\left[D^{\alpha} D^{\beta} U\right]_{-}\left(\left[D^{\gamma} U\right]_{-}\left[D_{\beta} U\right]_{-}\left[G^{\mu \nu}\right]_{+}-\left[G^{\mu \nu}\right]_{+}\left[D_{\beta} U\right]_{-}\left[D^{\gamma} U\right]_{-}\right)\right) \epsilon_{\alpha \gamma \mu \nu}, \\
\operatorname{Tr}\left(\left[D^{\alpha} D_{\beta} U\right]_{-}\left(\left[D^{\gamma} U\right]_{-}\left[D^{\delta} U\right]_{-}\left[G^{\beta \nu}\right]_{+}-\left[G^{\beta \nu}\right]_{+}\left[D^{\delta} U\right]_{-}\left[D^{\gamma} U\right]_{-}\right)\right) \epsilon_{\alpha \gamma \delta \nu}, \\
\operatorname{Tr}\left(\left[D^{\alpha} D_{\beta} U\right]_{-}\left[D^{\gamma} U\right]_{-}\left[G^{\beta \nu}\right]_{+}\left[D^{\delta} U\right]_{-}\right) \epsilon_{\alpha \gamma \delta \nu},
\end{gathered}
$$

from $3 b)$

$$
\begin{aligned}
& \operatorname{Tr}\left(\left[D_{\alpha} D^{\alpha} U\right]_{-}\left(\left[D_{\mu} U\right]_{-}\left[D_{\nu} U\right]_{-}\left[H^{\mu \nu}\right]_{+}+\left[H^{\mu \nu}\right]_{+}\left[D_{\nu} U\right]_{-}\left[D_{\mu} U\right]_{-}\right)\right), \\
& \operatorname{Tr}\left(\left[D_{\mu} D_{\beta} U\right]_{-}\left(\left[D^{\beta} U\right]_{-}\left[D_{\nu} U\right]_{-}\left[H^{\mu \nu}\right]_{+}+\left[H^{\mu \nu}\right]_{+}\left[D_{\nu} U\right]_{-}\left[D^{\beta} U\right]_{-}\right)\right),
\end{aligned}
$$




$$
\begin{aligned}
& \operatorname{Tr}\left(\left[D_{\mu} D_{\beta} U\right]_{-}\left(\left[D_{\nu} U\right]_{-}\left[D^{\beta} U\right]_{-}\left[H^{\mu \nu}\right]_{+}+\left[H^{\mu \nu}\right]_{+}\left[D^{\beta} U\right]_{-}\left[D_{\nu} U\right]_{-}\right)\right), \\
& \operatorname{Tr}\left(\left[D_{\mu} D_{\beta} U\right]_{-}\left(\left[D_{\nu} U\right]_{-}\left[H^{\mu \nu}\right]_{+}\left[D^{\beta} U\right]_{-}+\left[D^{\beta} U\right]_{-}\left[H^{\mu \nu}\right]_{+}\left[D_{\nu} U\right]_{-}\right)\right),
\end{aligned}
$$

from 4a)

$$
\begin{aligned}
& \operatorname{Tr}\left(\left[D_{\alpha} D^{\alpha} U\right]_{-}\left(\left[D_{\mu} U\right]_{-}\left[D_{\nu} G^{\mu \nu}\right]_{+}-\left[D_{\nu} G^{\mu \nu}\right]_{+}\left[D_{\mu} U\right]_{-}\right)\right), \\
& \operatorname{Tr}\left(\left[D_{\mu} D^{\beta} U\right]_{-}\left(\left[D_{\beta} U\right]_{-}\left[D_{\nu} G^{\mu \nu}\right]_{+}-\left[D_{\nu} G^{\mu \nu}\right]_{+}\left[D_{\beta} U\right]_{-}\right)\right), \\
& \operatorname{Tr}\left(\left[D_{\mu} D^{\beta} U\right]_{-}\left(\left[D_{\nu} U\right]_{-}\left[D_{\beta} G^{\mu \nu}\right]_{+}-\left[D_{\beta} G^{\mu \nu}\right]_{+}\left[D_{\nu} U\right]_{-}\right)\right),
\end{aligned}
$$

from $4 b$ )

$$
\begin{aligned}
& \operatorname{Tr}\left(\left[D_{\alpha} D^{\alpha} U\right]_{-}\left(\left[D^{\beta} U\right]_{-}\left[D^{\gamma} H^{\mu \nu}\right]_{+}+\left[D^{\gamma} H^{\mu \nu}\right]_{+}\left[D^{\beta} U\right]_{-}\right)\right) \epsilon_{\beta \gamma \mu \nu}, \\
& \operatorname{Tr}\left(\left[D^{\alpha} D^{\beta} U\right]_{-}\left(\left[D_{\beta} U\right]_{-}\left[D^{\gamma} H^{\mu \nu}\right]_{+}+\left[D^{\gamma} H^{\mu \nu}\right]_{+}\left[D_{\beta} U\right]_{-}\right)\right) \epsilon_{\alpha \gamma \mu \nu}, \\
& \operatorname{Tr}\left(\left[D^{\alpha} D^{\beta} U\right]_{-}\left(\left[D^{\gamma} U\right]_{-}\left[D_{\beta} H^{\mu \nu}\right]_{+}+\left[D_{\beta} H^{\mu \nu}\right]_{+}\left[D^{\gamma} U\right]_{-}\right)\right) \epsilon_{\alpha \gamma \mu \nu}, \\
& \operatorname{Tr}\left(\left[D^{\alpha} D_{\beta} U\right]_{-}\left(\left[D^{\gamma} U\right]_{-}\left[D^{\delta} H^{\beta \nu}\right]_{+}+\left[D^{\delta} H^{\beta \nu}\right]_{+}\left[D^{\gamma} U\right]_{-}\right)\right) \epsilon_{\alpha \gamma \delta \nu},
\end{aligned}
$$

from 7a)

$$
\begin{gathered}
\operatorname{Tr}\left(\left[G^{\mu \nu}\right]_{+}\left(\left[D_{\mu} U\right]_{-}\left[D_{\nu} U\right]_{-}\left[D_{\alpha} U\right]_{-}\left[D^{\alpha} U\right]_{-}-\left[D_{\alpha} U\right]_{-}\left[D^{\alpha} U\right]_{-}\left[D_{\nu} U\right]_{-}\left[D_{\mu} U\right]_{-}\right)\right), \\
\operatorname{Tr}\left(\left[G^{\mu \nu}\right]_{+}\left(\left[D_{\mu} U\right]_{-}\left[D_{\alpha} U\right]_{-}\left[D_{\nu} U\right]_{-}\left[D^{\alpha} U\right]_{-}-\left[D_{\alpha} U\right]_{-}\left[D_{\nu} U\right]_{-}\left[D^{\alpha} U\right]_{-}\left[D_{\mu} U\right]_{-}\right)\right), \\
\operatorname{Tr}\left(\left[G^{\mu \nu}\right]_{+}\left[D_{\mu} U\right]_{-}\left[D_{\alpha} U\right]_{-}\left[D^{\alpha} U\right]_{-}\left[D_{\nu} U\right]_{-}\right), \\
\operatorname{Tr}\left(\left[G^{\mu \nu}\right]_{+}\left[D_{\alpha} U\right]_{-}\left[D_{\mu} U\right]_{-}\left[D_{\nu} U\right]_{-}\left[D^{\alpha} U\right]_{-}\right),
\end{gathered}
$$

and from $7 b$ )

$$
\begin{aligned}
& \operatorname{Tr}\left(\left[H^{\mu \nu}\right]_{+}\left(\left[D^{\alpha} U\right]_{-}\left[D^{\beta} U\right]_{-}\left[D_{\gamma} U\right]_{-}\left[D^{\gamma} U\right]_{-}+\left[D_{\gamma} U\right]_{-}\left[D^{\gamma} U\right]_{-}\left[D^{\beta} U\right]_{-}\left[D^{\alpha} U\right]_{-}\right)\right) \epsilon_{\mu \nu \alpha \beta}, \\
& \operatorname{Tr}\left(\left[H^{\mu \nu}\right]_{+}\left(\left[D^{\alpha} U\right]_{-}\left[D_{\gamma} U\right]_{-}\left[D^{\beta} U\right]_{-}\left[D^{\gamma} U\right]_{-}+\left[D_{\gamma} U\right]_{-}\left[D^{\beta} U\right]_{-}\left[D^{\gamma} U\right]_{-}\left[D^{\alpha} U\right]_{-}\right)\right) \epsilon_{\mu \nu \alpha \beta}
\end{aligned}
$$




$$
\begin{aligned}
& \operatorname{Tr}\left(\left[H^{\mu \nu}\right]_{+}\left(\left[D_{\nu} U\right]_{-}\left[D^{\beta} U\right]_{-}\left[D^{\gamma} U\right]_{-}\left[D^{\delta} U\right]_{-}+\left[D^{\delta} U\right]_{-}\left[D^{\gamma} U\right]_{-}\left[D^{\beta} U\right]_{-}\left[D_{\nu} U\right]_{-}\right)\right) \epsilon_{\mu \beta \gamma \delta}, \\
& \operatorname{Tr}\left(\left[H^{\mu \nu}\right]_{+}\left(\left[D^{\beta} U\right]_{-}\left[D_{\nu} U\right]_{-}\left[D^{\gamma} U\right]_{-}\left[D^{\delta} U\right]_{-}+\left[D^{\delta} U\right]_{-}\left[D^{\gamma} U\right]_{-}\left[D_{\nu} U\right]_{-}\left[D^{\beta} U\right]_{-}\right)\right) \epsilon_{\mu \beta \gamma \delta} .
\end{aligned}
$$

In this group the set Eqs. (119), (120), and (121) are related by the trace relations of Sec. IIE6, and we use that result to eliminate Eq. (121). Also a number of terms are related by the epsilon relations of Sec. IIE7. In particular there is one relation among terms of each of the sets Eqs. (112), (114), and (117), Eqs. (111), (113), (115), and (116) and Eqs. (125), (126), (127), and (128) which we use to eliminate say Eqs. (114), (116), and (128). Just as in the discussion following Eq. (58) we here also keep the equation of motion terms, since they can be eliminated later via field transformations, and use these relations to eliminate other terms. There are two relations among the set Eqs. (133), (134), (135), and (136) which we use to eliminate Eqs. (135) and (136).

Again we can write down directly from Eq. (82) the multiple trace terms. Many possible terms vanish because for $\mu \neq \nu$ we have $\operatorname{Tr}\left(\left[\chi^{\mu \nu}\right]_{ \pm}\right)=\operatorname{Tr}\left(\left[D_{\alpha} \chi^{\mu \nu}\right]_{+}\right)=0$, as can be shown by explicit evaluation using the definitions Eq. (20). Because of these relations, and also $\operatorname{Tr}\left(\left[D_{\alpha} D_{\beta} U\right]_{-}\right)=\operatorname{Tr}\left(\left[D_{\alpha} U\right]_{-}\right)=0$, we obtain no terms from $\left.\left.3 \mathrm{a}\right), 4 \mathrm{a}\right)$, or $\left.4 \mathrm{~b}\right)$, and we get from $3 \mathrm{~b}$ )

$$
\begin{aligned}
& \operatorname{Tr}\left(\left[D_{\alpha} D^{\alpha} U\right]_{-}\left[D_{\mu} U\right]_{-}\right) \operatorname{Tr}\left(\left[D_{\nu} U\right]_{-}\left[H^{\mu \nu}\right]_{+}\right), \\
& \operatorname{Tr}\left(\left[D_{\mu} D_{\beta} U\right]_{-}\left[D^{\beta} U\right]_{-}\right) \operatorname{Tr}\left(\left[D_{\nu} U\right]_{-}\left[H^{\mu \nu}\right]_{+}\right), \\
& \operatorname{Tr}\left(\left[D_{\mu} D_{\beta} U\right]_{-}\left[D_{\nu} U\right]_{-}\right) \operatorname{Tr}\left(\left[D^{\beta} U\right]_{-}\left[H^{\mu \nu}\right]_{+}\right), \\
& \operatorname{Tr}\left(\left[D_{\mu} D_{\beta} U\right]_{-}\left[H^{\mu \nu}\right]_{+}\right) \operatorname{Tr}\left(\left[D^{\beta} U\right]_{-}\left[D_{\nu} U\right]_{-}\right),
\end{aligned}
$$

from 7a)

$$
\begin{gathered}
\operatorname{Tr}\left(\left[G^{\mu \nu}\right]_{+}\left[D_{\alpha} U\right]_{-}\right) \operatorname{Tr}\left(\left[D^{\alpha} U\right]_{-}\left[D_{\mu} U\right]_{-}\left[D_{\nu} U\right]_{-}\right), \\
\operatorname{Tr}\left(\left[G^{\mu \nu}\right]_{+}\left[D_{\mu} U\right]_{-}\left[D_{\nu} U\right]_{-}\right) \operatorname{Tr}\left(\left[D_{\alpha} U\right]_{-}\left[D^{\alpha} U\right]_{-}\right), \\
\operatorname{Tr}\left(\left[G^{\mu \nu}\right]_{+}\left(\left[D_{\mu} U\right]_{-}\left[D_{\alpha} U\right]_{-}-\left[D_{\alpha} U\right]_{-}\left[D_{\mu} U\right]_{-}\right)\right) \operatorname{Tr}\left(\left[D^{\alpha} U\right]_{-}\left[D_{\nu} U\right]_{-}\right),
\end{gathered}
$$

and from $7 b$ )

$$
\begin{gathered}
\operatorname{Tr}\left(\left[H^{\mu \nu}\right]_{+}\left[D^{\alpha} U\right]_{-}\right) \operatorname{Tr}\left(\left[D^{\beta} U\right]_{-}\left[D_{\gamma} U\right]_{-}\left[D^{\gamma} U\right]_{-}\right) \epsilon_{\mu \nu \alpha \beta} \\
\operatorname{Tr}\left(\left[H^{\mu \nu}\right]_{+}\left(\left[D^{\alpha} U\right]_{-}\left[D^{\gamma} U\right]_{-}+\left[D^{\gamma} U\right]_{-}\left[D^{\alpha} U\right]_{-}\right)\right) \operatorname{Tr}\left(\left[D^{\beta} U\right]_{-}\left[D_{\gamma} U\right]_{-}\right) \epsilon_{\mu \nu \alpha \beta} .
\end{gathered}
$$




\section{Terms with two $D_{\mu}$ 's and two $\chi^{\mu \nu}$ 's}

Consider now terms of the third level in the hierarchy, those containing two $D_{\mu}$ 's and two $\chi^{\mu \nu}$ 's. The $\chi^{\mu \nu}$ 's can be just $\chi$ if $\mu=\nu$, or can be the full $\chi^{\mu \nu}$ involving the $F^{\mu \nu}$ 's if $\mu \neq \nu$, or there can be one of each. Because of these different possibilities, and the fact that each $\chi^{\mu \nu}$ has its own parity and charge conjugation quantum numbers there are so many options that it is much more difficult in this case to develop a general result and simply evaluate it, as was done in the previous two sections. We will however proceed as far as possible in general, but will be forced in the end to simply enumerate the possibilities.

Note that we will have to distinguish between the two $\chi^{\mu \nu}$ 's. To do this we will call one $\chi$ and the other $\tilde{\chi}$. To simplify the notation we will also drop the Lorentz indices on $\chi$ until the end.

We can then write the possible terms directly in terms of the building blocks $[A]_{ \pm}$as

$$
\begin{aligned}
\text { 1) } & {[D \chi]_{ \pm}[D \tilde{\chi}]_{ \pm} } \\
\text {2a) } & {[D U]_{-}[D \chi]_{ \pm}[\tilde{\chi}]_{ \pm} } \\
\text {2b) } & {[D U]_{-}[D \tilde{\chi}]_{ \pm}[\chi]_{ \pm} } \\
3) & {[D U]_{-}[D U]_{-}[\chi]_{ \pm}[\tilde{\chi}]_{ \pm} }
\end{aligned}
$$

In these expressions we must take all independent orders, and of course eventually put the Lorentz indices back in and take all possible contractions. The \pm signs on the $\chi$ and $\tilde{\chi}$ terms are uncorrelated, so that all combinations must be included.

To obtain these expressions we have already systematically used the total derivative argument of Sec. IIE 1 to remove any $D^{2}$ 's. However this procedure may have hidden some equation of motion terms in this set, which we want to extract. For example, moving the $D$ acting on $\chi$ in 2 a) over to the $D U$ using the total derivative argument could generate a $\left[D_{\alpha} D^{\alpha} U\right]_{-}$term. There are some subtleties in such a transformation however. So far we have eliminated terms high in the hierarchy in favor of those lower down, i.e. in this case we eliminate those with more $D$ 's acting on a single factor in favor of those with fewer $D$ 's. Since we always keep the most general set of terms at the lower level, it is never necessary to actually work out explicitly the relation used to eliminate the higher level term. Here however we are asking whether a specific lower level term can be eliminated in favor of a higher level one. Thus to be sure one is not over or under counting the independent terms it will be necessary here to work out the transformation explicitly.

To do this consider the total derivative $\partial_{\mu}\left(\left[D_{\nu} U\right]_{-}[\chi]_{ \pm}[\tilde{\chi}]_{ \pm}\right)$, where the argument of the derivative is understood to be a single or multiple trace. Since total derivatives can be dropped in the Lagrangian, this can be treated as if it were effectively zero. Thus using the results of Sec. IIE 1 and particularly Eq. (24) we find

$$
\begin{aligned}
0=[ & \left.D_{\mu} D_{\nu} U\right]_{-}[\chi]_{ \pm}[\tilde{\chi}]_{ \pm} \\
& +\left[D_{\nu} U\right]_{-}\left[D_{\mu} \chi\right]_{ \pm}[\tilde{\chi}]_{ \pm}+\left[D_{\nu} U\right]_{-}[\chi]_{ \pm}\left[D_{\mu} \tilde{\chi}\right]_{ \pm} \\
& + \text {terms of the class } 3),
\end{aligned}
$$

where again the traces have not been put in explicitly. The terms of the class 3) arise from the covariant derivative acting on the $U$ and $U^{\dagger}$ in the various factors $[A]_{ \pm}$together with some algebraic rearrangement to express everything in terms of the standard factors $[A]_{ \pm}$. 
Thus we see that we can express a particular sum of the terms from the classes 2a) and $2 \mathrm{~b})$ in terms of a class containing a double derivative term $\left[D_{\mu} D_{\nu} U\right]_{-}$. To get this particular sum we must take a new basis for the terms of $2 \mathrm{a}$ ) and $2 \mathrm{~b}$ ) consisting of the sums and differences of terms. When this is done we can use this double derivative term instead of the sum if we wish, but we must keep the remaining (difference) terms from 2a) and $2 \mathrm{~b}$ ). Note that we could have started this discussion with alternative ordering, $\chi \leftrightarrow \tilde{\chi}$, but since we always include all orders of the factors, this gives the same results.

This means that in the general result of Eq. (146) we may use instead of $2 \mathrm{a}$ ) and $2 \mathrm{~b}$ )

$$
\begin{array}{ll}
2 a)^{\prime} & {[D U]_{-}\left([D \chi]_{ \pm}[\tilde{\chi}]_{ \pm}-[\chi]_{ \pm}[D \tilde{\chi}]_{ \pm}\right),} \\
2 b)^{\prime} & {\left[D_{\mu} D_{\nu} U\right]_{-}[\chi]_{ \pm}[\tilde{\chi}]_{ \pm}}
\end{array}
$$

where as usual, all orderings must be taken and the \pm signs are not correlated.

Observe that everything done so far applies to multiple traces just as to single traces. One sees, by following through the arguments leading to Eq. (148), that if we start in 2a) and $2 \mathrm{~b}$ ) with say, the trace of the first two factors times the trace of the third, then we will also get the trace of the first two factors times the trace of the third in each of the terms making up 2a)' and $2 \mathrm{~b})^{\prime}$.

Finally it is possible to apply essentially the same argument to 1) of Eq. (146) and show that we can use either of the alternative forms

$$
\begin{aligned}
1)^{\prime} & {[\chi]_{ \pm}\left[D_{\mu} D_{\nu} \tilde{\chi}\right]_{ \pm}, } \\
1)^{\prime \prime} & {\left[D_{\mu} D_{\nu} \chi\right]_{ \pm}[\tilde{\chi}]_{ \pm} }
\end{aligned}
$$

Next we must ensure that parity and charge conjugation are satisfied. Recall that effectively under parity $[A]_{ \pm} \rightarrow \pm(-1)^{p} U^{\dagger}[A]_{ \pm} U$ with $p$ being the intrinsic parity of the operator $A$. There is also the extra factor of $(-1)^{\epsilon}$, where $\epsilon$ counts the number of $\epsilon_{\alpha \beta \gamma \delta}$ 's in the term. Let $p$ and $\tilde{p}$ be the intrinsic parities of $\chi$ and $\tilde{\chi}$ and let $(-1)^{s}$ here account for the product of \pm signs coming from the $[\chi]_{ \pm}$and $[\tilde{\chi}]_{ \pm}$factors only, e. g. $s=0$ for $[\chi]_{+}[\tilde{\chi}]_{+}$and $s=1$ for $[\chi]_{+}[\tilde{\chi}]_{-}$. Thus we get a parity invariant term by multiplying the forms 1) and 3) by $\left(1+(-1)^{s+p+\tilde{p}+\epsilon}\right)$ and the forms $\left.2 \mathrm{a}\right)^{\prime}$ and $\left.2 \mathrm{~b}\right)^{\prime}$ by $\left(1-(-1)^{s+p+\tilde{p}+\epsilon}\right)$.

To ensure charge conjugation invariance we must add $(-1)^{c+\tilde{c}}$ times the major factors in reverse order, where $c$ and $\tilde{c}$ are the intrinsic charge conjugation quantum numbers of $\chi$ and $\tilde{\chi}$. For terms of type 1 ) and the multiple trace terms from type $2 \mathrm{a})^{\prime}$ and $\left.2 \mathrm{~b}\right)^{\prime}$ with only two factors in the trace the cyclic property of the trace means that this amounts to multiplying by the overall factor $\left(1+(-1)^{c+\tilde{c}}\right)$. For the others the reversed term has to be added explicitly, though in many cases adding such a term makes different initial starting orders for the factors give the same result.

We can now summarize the general form for the two $D_{\mu}$ and two $\chi^{\mu \nu}$ terms as follows.

1) $\left(1+(-1)^{s+p+\tilde{p}+\epsilon}\right)\left(1+(-1)^{c+\tilde{c}}\right)\left[D_{\mu} \chi^{\alpha \beta}\right]_{ \pm}\left[D_{\nu} \tilde{\chi}^{\gamma \delta}\right]_{ \pm}$,

$2 a)^{\prime} \quad\left(1-(-1)^{s+p+\tilde{p}+\epsilon}\right)\left(\left[D_{\mu} U\right]_{-}\left(\left[D_{\nu} \chi^{\alpha \beta}\right]_{ \pm}\left[\tilde{\chi}^{\gamma \delta}\right]_{ \pm}-\left[\chi^{\alpha \beta}\right]_{ \pm}\left[D_{\nu} \tilde{\chi}^{\gamma \delta}\right]_{ \pm}\right)+(-1)^{c+\tilde{c}}(\mathrm{rev})\right)$,

$2 b)^{\prime} \quad\left(1-(-1)^{s+p+\tilde{p}+\epsilon}\right)\left(\left[D_{\mu} D_{\nu} U\right]_{-}\left[\chi^{\alpha \beta}\right]_{ \pm}\left[\tilde{\chi}^{\gamma \delta}\right]_{ \pm}+(-1)^{c+\tilde{c}}(\mathrm{rev})\right)$,

3) $\left(1+(-1)^{s+p+\tilde{p}+\epsilon}\right)\left(\left[D_{\mu} U\right]_{-}\left[D_{\nu} U\right]_{-}\left[\chi^{\alpha \beta}\right]_{ \pm}\left[\tilde{\chi}^{\gamma \delta}\right]_{ \pm}+(-1)^{c+\tilde{c}}(\mathrm{rev})\right)$. 
In these expressions one must contract the Lorentz indices in all possible ways, including perhaps contracting with an $\epsilon_{\alpha \beta \gamma \delta}$, must take all possible independent orders for the factors, and in the end must take a trace. The \pm signs are not correlated, though the $(-1)^{s}$ in the parity factor will enforce a correlation for given values of the other quantum numbers. The indication 'rev' means to take the major factors in reverse order. For multiple traces, one must in addition take all possible combinations of different traces. Just as in the previous section the evaluation of the result, particularly the multiple trace part, is simplified by the relations $\operatorname{Tr}\left(\left[D_{\mu} D_{\nu} U\right]_{-}\right)=\operatorname{Tr}\left(\left[D_{\mu} U\right]_{-}\right)=0$. Also for $\mu \neq \nu \operatorname{Tr}\left(\left[\chi^{\mu \nu}\right]_{ \pm}\right)=\left[\chi^{\mu \nu}\right]_{-}=$ $\operatorname{Tr}\left(\left[D_{\alpha} \chi^{\mu \nu}\right]_{+}\right)=0$. Furthermore $\left[D_{\alpha} \chi^{\mu \nu}\right]_{-}$can be expressed in terms of other quantities lower in the hierarchy which have been kept, and so can be dropped.

We now proceed to evaluate this general expression. There are three main cases, namely, I) $\chi$ and $\tilde{\chi}$ both simple $\chi^{\prime}$ 's, II) one a $\chi$ and the other a $\chi^{\mu \nu}$ with $\mu \neq \nu$, and III) both $\chi^{\mu \nu}$ s. Furthermore each $\chi^{\mu \nu}$ can be a $G^{\mu \nu}$ or an $H^{\mu \nu}$. We will evaluate the single and multiple traces together, as that seems simplest here, and will always drop irrelevant overall numerical factors.

Consider case I) where both $\chi$ and $\tilde{\chi}$ are simple $\chi$ 's. The quantum numbers $p=\tilde{p}=c=$ $\tilde{c}=\epsilon=0$ so that $s=0$ for terms from 1) and 3) and $s=1$ for those from $2 \mathrm{a})^{\prime}$ and $\left.2 \mathrm{~b}\right)^{\prime}$. We obtain from 1)

$$
\begin{gathered}
\operatorname{Tr}\left(\left[D_{\mu} \chi\right]_{+}\left[D^{\mu} \chi\right]_{+}\right), \\
\operatorname{Tr}\left(\left[D_{\mu} \chi\right]_{-}\left[D^{\mu} \chi\right]_{-}\right), \\
\operatorname{Tr}\left(\left[D_{\mu} \chi\right]_{+}\right) \operatorname{Tr}\left(\left[D^{\mu} \chi\right]_{+}\right), \\
\operatorname{Tr}\left(\left[D_{\mu} \chi\right]_{-}\right) \operatorname{Tr}\left(\left[D^{\mu} \chi\right]_{-}\right),
\end{gathered}
$$

from $2 \mathrm{a})^{\prime}$

$$
\begin{gathered}
\operatorname{Tr}\left(\left[D_{\mu} U\right]_{-}\left(\left[D^{\mu} \chi\right]_{+}[\chi]_{-}-[\chi]_{+}\left[D^{\mu} \chi\right]_{-}+[\chi]_{-}\left[D^{\mu} \chi\right]_{+}-\left[D^{\mu} \chi\right]_{-}[\chi]_{+}\right)\right) \\
\operatorname{Tr}\left(\left[D_{\mu} U\right]_{-}\left[D^{\mu} \chi\right]_{+}\right) \operatorname{Tr}\left([\chi]_{-}\right)-\operatorname{Tr}\left(\left[D_{\mu} U\right]_{-}[\chi]_{+}\right) \operatorname{Tr}\left(\left[D^{\mu} \chi\right]_{-}\right) \\
\operatorname{Tr}\left(\left[D_{\mu} U\right]_{-}\left[D^{\mu} \chi\right]_{-}\right) \operatorname{Tr}\left([\chi]_{+}\right)-\operatorname{Tr}\left(\left[D_{\mu} U\right]_{-}[\chi]_{-}\right) \operatorname{Tr}\left(\left[D^{\mu} \chi\right]_{+}\right)
\end{gathered}
$$

from $2 b)^{\prime}$

$$
\begin{gathered}
\operatorname{Tr}\left(\left[D_{\mu} D^{\mu} U\right]_{-}\left([\chi]_{+}[\chi]_{-}+[\chi]_{-}[\chi]_{+}\right)\right), \\
\operatorname{Tr}\left(\left[D_{\mu} D^{\mu} U\right]_{-}[\chi]_{+}\right) \operatorname{Tr}\left([\chi]_{-}\right), \\
\operatorname{Tr}\left(\left[D_{\mu} D^{\mu} U\right]_{-}[\chi]_{-}\right) \operatorname{Tr}\left([\chi]_{+}\right),
\end{gathered}
$$


and from 3)

$$
\begin{gathered}
\operatorname{Tr}\left(\left[D_{\mu} U\right]_{-}\left[D^{\mu} U\right]_{-}[\chi]_{+}[\chi]_{+}\right), \\
\operatorname{Tr}\left(\left[D_{\mu} U\right]_{-}[\chi]_{+}\left[D^{\mu} U\right]_{-}[\chi]_{+}\right), \\
\operatorname{Tr}\left(\left[D_{\mu} U\right]_{-}\left[D^{\mu} U\right]_{-}[\chi]_{-}[\chi]_{-}\right), \\
\operatorname{Tr}\left(\left[D_{\mu} U\right]_{-}[\chi]_{-}\left[D^{\mu} U\right]_{-}[\chi]_{-}\right), \\
\operatorname{Tr}\left(\left[D_{\mu} U\right]_{-}\left[D^{\mu} U\right]_{-}[\chi]_{+}\right) \operatorname{Tr}\left([\chi]_{+}\right), \\
\operatorname{Tr}\left(\left[D_{\mu} U\right]_{-}\left[D^{\mu} U\right]_{-}[\chi]_{-}\right) \operatorname{Tr}\left([\chi]_{-}\right), \\
\operatorname{Tr}\left(\left[D_{\mu} U\right]_{-}\left[\left[D^{\mu} U\right]_{-}\right) \operatorname{Tr}\left([\chi]_{+}\right) \operatorname{Tr}\left([\chi]_{+}\right),\right. \\
\left.\operatorname{Tr}\left(\left[D_{\mu} U\right]_{-}\right),\left[D^{\mu} U\right]_{-}\right) \operatorname{Tr}\left([\chi]_{-}[\chi]_{-}\right), \\
\operatorname{Tr}\left(\left[D_{\mu} U\right]_{-}[\chi]_{+}\right) \operatorname{Tr}\left(\left[D^{\mu} U\right]_{-}[\chi]_{+}\right), \\
\operatorname{Tr}\left([\chi]_{-}\right) \operatorname{Tr}\left([\chi]_{-}\right) \operatorname{Tr}\left(\left[D^{\mu} U\right]_{-}[\chi]_{-}\right),
\end{gathered}
$$

Of these there are two sets of terms which consist of all permutations of factors, Eqs. (161) and (162) and Eqs. (163) and (164). Within each set the terms are thus related by the trace relations of Sec. IIE6 which we use to eliminate Eqs. (162) and (164).

Now consider case II) where say $\chi$ is the simple $\chi$ and $\tilde{\chi}$ is one of $G^{\mu \nu}$ or $H^{\mu \nu}$. Now $p=c=0$, with $\tilde{p}=0$ and $\tilde{c}=1$ for $G^{\gamma \delta}$ or $\tilde{p}=1$ and $\tilde{c}=0$ for $H^{\gamma \delta}$. There are now four indices so it is possible to have an $\epsilon_{\mu \nu \gamma \delta}$ factor. Observe that $\left.2 \mathrm{~b}\right)^{\prime}$, and also 1 ), if we use the equivalent form 1)' of Eq. (149), are symmetric in the interchange $\mu \leftrightarrow \nu$ and hence they will vanish when contracted either with $\tilde{\chi}^{\mu \nu}$ or $\epsilon_{\mu \nu \gamma \delta}$. Thus non zero terms come only from $2 \mathrm{a})^{\prime}$ and 3 ).

We then obtain from $2 \mathrm{a})^{\prime}$ without an $\epsilon_{\mu \nu \gamma \delta}$ factor

$$
\operatorname{Tr}\left(\left[D_{\mu} U\right]_{-}\left(\left[D_{\nu} \chi\right]_{-}\left[G^{\mu \nu}\right]_{+}-[\chi]_{-}\left[D_{\nu} G^{\mu \nu}\right]_{+}-\left[G^{\mu \nu}\right]_{+}\left[D_{\nu} \chi\right]_{-}+\left[D_{\nu} G^{\mu \nu}\right]_{+}[\chi]_{-}\right)\right),
$$




$$
\begin{gathered}
\operatorname{Tr}\left(\left[D_{\mu} U\right]_{-}\left(\left[D_{\nu} \chi\right]_{+}\left[H^{\mu \nu}\right]_{+}-[\chi]_{+}\left[D_{\nu} H^{\mu \nu}\right]_{+}+\left[H^{\mu \nu}\right]_{+}\left[D_{\nu} \chi\right]_{+}-\left[D_{\nu} H^{\mu \nu}\right]_{+}[\chi]_{+}\right)\right) \\
\operatorname{Tr}\left(\left[D_{\mu} U\right]_{-}\left[H^{\mu \nu}\right]_{+}\right) \operatorname{Tr}\left(\left[D_{\nu} \chi\right]_{+}\right)-\operatorname{Tr}\left(\left[D_{\mu} U\right]_{-}\left[D_{\nu} H^{\mu \nu}\right]_{+}\right) \operatorname{Tr}\left([\chi]_{+}\right),
\end{gathered}
$$

and with an $\epsilon_{\mu \nu \gamma \delta}$ factor

$$
\begin{gathered}
\operatorname{Tr}\left(\left[D^{\mu} U\right]_{-}\left(\left[D^{\nu} \chi\right]_{+}\left[G^{\gamma \delta}\right]_{+}-[\chi]_{+}\left[D^{\nu} G^{\gamma \delta}\right]_{+}-\left[G^{\gamma \delta}\right]_{+}\left[D^{\nu} \chi\right]_{+}+\left[D^{\nu} G^{\gamma \delta}\right]_{+}[\chi]_{+}\right)\right) \epsilon_{\mu \nu \gamma \delta} \\
\operatorname{Tr}\left(\left[D^{\mu} U\right]_{-}\left(\left[D^{\nu} \chi\right]_{-}\left[H^{\gamma \delta}\right]_{+}-[\chi]_{-}\left[D^{\nu} H^{\gamma \delta}\right]_{+}+\left[H^{\gamma \delta}\right]_{+}\left[D^{\nu} \chi\right]_{-}\left[D^{\nu} H^{\gamma \delta}\right]_{+}[\chi]_{-}\right)\right) \epsilon_{\mu \nu \gamma \delta} \\
\left(\operatorname{Tr}\left(\left[D^{\mu} U\right]_{-}\left[H^{\gamma \delta}\right]_{+}\right) \operatorname{Tr}\left(\left[D^{\nu} \chi\right]_{-}\right)-\operatorname{Tr}\left(\left[D^{\mu} U\right]_{-}\left[D^{\nu} H^{\gamma \delta}\right]_{+}\right) \operatorname{Tr}\left([\chi]_{-}\right)\right) \epsilon_{\mu \nu \gamma \delta}
\end{gathered}
$$

From 3) without an $\epsilon_{\mu \nu \gamma \delta}$ factor we get

$$
\begin{gathered}
\operatorname{Tr}\left(\left[D_{\mu} U\right]_{-}\left[D_{\nu} U\right]_{-}\left([\chi]_{+}\left[G^{\mu \nu}\right]_{+}+\left[G^{\mu \nu}\right]_{+}[\chi]_{+}\right)\right) \\
\operatorname{Tr}\left(\left[D_{\mu} U\right]_{-}[\chi]_{+}\left[D_{\nu} U\right]_{-}\left[G^{\mu \nu}\right]_{+}\right) \\
\operatorname{Tr}\left(\left[D_{\mu} U\right]_{-}\left[D_{\nu} U\right]_{-}\left[G^{\mu \nu}\right]_{+}\right) \operatorname{Tr}\left([\chi]_{+}\right), \\
\operatorname{Tr}\left(\left[D_{\mu} U\right]_{-}\left[D_{\nu} U\right]_{-}\left([\chi]_{-}\left[H^{\mu \nu}\right]_{+}-\left[H^{\mu \nu}\right]_{+}[\chi]_{-}\right)\right), \\
\operatorname{Tr}\left(\left[D_{\mu} U\right]_{-}\left[H^{\mu \nu}\right]_{+}\right) \operatorname{Tr}\left(\left[D_{\nu} U\right]_{-}[\chi]_{-}\right),
\end{gathered}
$$

and with an $\epsilon_{\mu \nu \gamma \delta}$ factor

$$
\begin{gathered}
\operatorname{Tr}\left(\left[D^{\mu} U\right]_{-}\left[D^{\nu} U\right]_{-}\left([\chi]_{-}\left[G^{\gamma \delta}\right]_{+}+\left[G^{\gamma \delta}\right]_{+}[\chi]_{-}\right)\right) \epsilon_{\mu \nu \gamma \delta} \\
\operatorname{Tr}\left(\left[D^{\mu} U\right]_{-}[\chi]_{-}\left[D^{\nu} U\right]_{-}\left[G^{\gamma \delta}\right]_{+}\right) \epsilon_{\mu \nu \gamma \delta} \\
\operatorname{Tr}\left(\left[D^{\mu} U\right]_{-}\left[D^{\nu} U\right]_{-}\left[G^{\gamma \delta}\right]_{+}\right) \operatorname{Tr}\left([\chi]_{-}\right) \epsilon_{\mu \nu \gamma \delta}, \\
\operatorname{Tr}\left(\left[D^{\mu} U\right]_{-}\left[D^{\nu} U\right]_{-}\left([\chi]_{+}\left[H^{\gamma \delta}\right]_{+}-\left[H^{\gamma \delta}\right]_{+}[\chi]_{+}\right)\right) \epsilon_{\mu \nu \gamma \delta} \\
\operatorname{Tr}\left(\left[D^{\mu} U\right]_{-}\left[H^{\gamma \delta}\right]_{+}\right) \operatorname{Tr}\left(\left[D^{\nu} U\right]_{-}[\chi]_{+}\right) \epsilon_{\mu \nu \gamma \delta} .
\end{gathered}
$$

Consider now the third case in which both $\chi$ and $\tilde{\chi}$ are $\chi^{\mu \nu}$ with $\mu \neq \nu$. Observe first that $s=0$ since all factors involving $\chi^{\mu \nu}$ 's are of the form $\left[\ldots \chi^{\mu \nu} \ldots\right]_{+}$. This means that when $p+\tilde{p}+\epsilon$ is even only 1) and 3) contribute while when it is odd only $2 \mathrm{a})^{\prime}$ and $\left.2 \mathrm{~b}\right)^{\prime}$ 
contribute. Furthermore for the $H H$ or $G G$ terms $p+\tilde{p}+\epsilon \rightarrow \epsilon$ and $c+\tilde{c}$ is even whereas for the $G H$ terms $p+\tilde{p}+\epsilon \rightarrow 1+\epsilon$ and $c+\tilde{c}$ is odd. All of the individual factors are traceless, so the only multiple traces possible must involve two factors and thus can come only from 3). Finally by considering various permutations we see that we get fewer terms at this level if we use the 1)' form instead of the 1) form.

We thus obtain from 1$)^{\prime}$

$$
\begin{aligned}
& \operatorname{Tr}\left(\left[G^{\alpha \beta}\right]_{+}\left[D_{\mu} D^{\mu} G_{\alpha \beta}\right]_{+}\right), \\
& \operatorname{Tr}\left(\left[G^{\alpha \beta}\right]_{+}\left[D_{\alpha} D^{\gamma} G_{\beta \gamma}\right]_{+}\right), \\
& \operatorname{Tr}\left(\left[H^{\alpha \beta}\right]_{+}\left[D_{\mu} D^{\mu} H_{\alpha \beta}\right]_{+}\right), \\
& \operatorname{Tr}\left(\left[H^{\alpha \beta}\right]_{+}\left[D_{\alpha} D^{\gamma} H_{\beta \gamma}\right]_{+}\right) .
\end{aligned}
$$

From 3) we get the following terms without an $\epsilon_{\alpha \beta \gamma \delta}$

$$
\begin{gathered}
\operatorname{Tr}\left(\left[D_{\mu} U\right]_{-}\left[D^{\mu} U\right]_{-}\left[G^{\alpha \beta}\right]_{+}\left[G_{\alpha \beta}\right]_{+}\right) \\
\operatorname{Tr}\left(\left[D_{\mu} U\right]_{-}\left[G^{\alpha \beta}\right]_{+}\left[D^{\mu} U\right]_{-}\left[G_{\alpha \beta}\right]_{+}\right) \\
\operatorname{Tr}\left(\left[D_{\alpha} U\right]_{-}\left[D^{\beta} U\right]_{-}\left[G^{\alpha \gamma}\right]_{+}\left[G_{\beta \gamma}\right]_{+}\right) \\
\operatorname{Tr}\left(\left[D_{\alpha} U\right]_{-}\left(\left[G^{\alpha \gamma}\right]_{+}\left[D^{\beta} U\right]_{-}\left[G_{\beta \gamma}\right]_{+}+\left[G_{\beta \gamma}\right]_{+}\left[D^{\beta} U\right]_{-}\left[G^{\alpha \gamma}\right]_{+}\right)\right) \\
\operatorname{Tr}\left(\left[D_{\alpha} U\right]_{-}\left[D^{\beta} U\right]_{-}\left[G_{\beta \gamma}\right]_{+}\left[G^{\alpha \gamma}\right]_{+}\right) \\
\operatorname{Tr}\left(\left[D_{\mu} U\right]_{-}\left[D^{\mu} U\right]_{-}\right) \operatorname{Tr}\left(\left[G^{\alpha \beta}\right]_{+}\left[G_{\alpha \beta}\right]_{+}\right) \\
\operatorname{Tr}\left(\left[D_{\mu} U\right]_{-}\left[G^{\alpha \beta}\right]_{+}\right) \operatorname{Tr}\left(\left[D^{\mu} U\right]_{-}\left[G_{\alpha \beta}\right]_{+}\right) \\
\operatorname{Tr}\left(\left[D_{\alpha} U\right]_{-}\left[G^{\alpha \gamma}\right]_{+}\right) \operatorname{Tr}\left(\left[D^{\beta} U\right]_{-}\left[G_{\beta \gamma}\right]_{+}\right) \\
\operatorname{Tr}\left(\left[D_{\alpha} U\right]_{-}\left[D^{\beta} U\right]_{-}\right) \operatorname{Tr}\left(\left[G^{\alpha \gamma}\right]_{+}\left[G_{\beta \gamma}\right]_{+}\right)
\end{gathered}
$$




$$
\begin{aligned}
& \operatorname{Tr}\left(\left[D^{\alpha} U\right]_{-}\left[G^{\beta \gamma}\right]_{+}\right) \operatorname{Tr}\left(\left[D_{\beta} U\right]_{-}\left[G_{\alpha \gamma}\right]_{+}\right), \\
& \operatorname{Tr}\left(\left[D_{\mu} U\right]_{-}\left[D^{\mu} U\right]_{-}\left[H^{\alpha \beta}\right]_{+}\left[H_{\alpha \beta}\right]_{+}\right), \\
& \operatorname{Tr}\left(\left[D_{\mu} U\right]_{-}\left[H^{\alpha \beta}\right]_{+}\left[D^{\mu} U\right]_{-}\left[H_{\alpha \beta}\right]_{+}\right), \\
& \operatorname{Tr}\left(\left[D_{\alpha} U\right]_{-}\left[D^{\beta} U\right]_{-}\left[H^{\alpha \gamma}\right]_{+}\left[H_{\beta \gamma}\right]_{+}\right), \\
& \operatorname{Tr}\left(\left[D_{\alpha} U\right]_{-}\left(\left[H^{\alpha \gamma}\right]_{+}\left[D^{\beta} U\right]_{-}\left[H_{\beta \gamma}\right]_{+}+\left[H_{\beta \gamma}\right]_{+}\left[D^{\beta} U\right]_{-}\left[H^{\alpha \gamma}\right]_{+}\right)\right) \\
& \operatorname{Tr}\left(\left[D_{\alpha} U\right]_{-}\left[D^{\beta} U\right]_{-}\left[H_{\beta \gamma}\right]_{+}\left[H^{\alpha \gamma}\right]_{+}\right), \\
& \operatorname{Tr}\left(\left[D_{\mu} U\right]_{-}\left[D^{\mu} U\right]_{-}\right) \operatorname{Tr}\left(\left[H^{\alpha \beta}\right]_{+}\left[H_{\alpha \beta}\right]_{+}\right), \\
& \operatorname{Tr}\left(\left[D_{\mu} U\right]_{-}\left[H^{\alpha \beta}\right]_{+}\right) \operatorname{Tr}\left(\left[D^{\mu} U\right]_{-}\left[H_{\alpha \beta}\right]_{+}\right), \\
& \operatorname{Tr}\left(\left[D_{\alpha} U\right]_{-}\left[D^{\beta} U\right]_{-}\right) \operatorname{Tr}\left(\left[H^{\alpha \gamma}\right]_{+}\left[H_{\beta \gamma}\right]_{+}\right), \\
& \operatorname{Tr}\left(\left[D_{\alpha} U\right]_{-}\left[H^{\alpha \gamma}\right]_{+}\right) \operatorname{Tr}\left(\left[D^{\beta} U\right]_{-}\left[H_{\beta \gamma}\right]_{+}\right), \\
& \operatorname{Tr}\left(\left[D^{\alpha} U\right]_{-}\left[H^{\beta \gamma}\right]_{+}\right) \operatorname{Tr}\left(\left[D_{\beta} U\right]_{-}\left[H_{\alpha \gamma}\right]_{+}\right) .
\end{aligned}
$$

Of these there are four sets of terms whose elements are related by the trace relations of Sec. IIE6, namely, Eqs. (193) and (194), Eqs. (195), (196), and (197), Eqs. (203) and (204), and Eqs. (205), (206), and (207). We use those relations to eliminate one term of each set, Eqs. (194), (196), (204), and (206).

The $\epsilon_{\alpha \beta \gamma \delta}$ terms from 3) are

$$
\begin{gathered}
\operatorname{Tr}\left(\left[D_{\mu} U\right]_{-}\left[D^{\mu} U\right]_{-}\left(\left[G^{\alpha \beta}\right]_{+}\left[H^{\gamma \delta}\right]_{+}-\left[H^{\gamma \delta}\right]_{+}\left[G^{\alpha \beta}\right]_{+}\right)\right) \epsilon_{\alpha \beta \gamma \delta}, \\
\operatorname{Tr}\left(\left[D^{\mu} U\right]_{-}\left[D^{\nu} U\right]_{-}\left(\left[G^{\alpha \beta}\right]_{+}\left[H_{\alpha}^{\gamma}\right]_{+}+\left[H_{\alpha}^{\gamma}\right]_{+}\left[G^{\alpha \beta}\right]_{+}\right)\right) \epsilon_{\mu \nu \beta \gamma}, \\
\operatorname{Tr}\left(\left[D^{\mu} U\right]_{-}\left[G^{\alpha \beta}\right]_{+}\left[D^{\nu} U\right]_{-}\left[H_{\alpha}^{\gamma}\right]_{+}\right) \epsilon_{\mu \nu \beta \gamma},
\end{gathered}
$$




$$
\begin{aligned}
& \operatorname{Tr}\left(\left[D^{\mu} U\right]_{-}\left(\left[D_{\alpha} U\right]_{-}\left[G^{\alpha \beta}\right]_{+}\left[H^{\gamma \delta}\right]_{+}-\left[H^{\gamma \delta}\right]_{+}\left[G^{\alpha \beta}\right]_{+}\left[D_{\alpha} U\right]_{-}\right)\right) \epsilon_{\mu \beta \gamma \delta}, \\
& \operatorname{Tr}\left(\left[D^{\mu} U\right]_{-}\left(\left[G^{\alpha \beta}\right]_{+}\left[D_{\alpha} U\right]_{-}\left[H^{\gamma \delta}\right]_{+}-\left[H^{\gamma \delta}\right]_{+}\left[D_{\alpha} U\right]_{-}\left[G^{\alpha \beta}\right]_{+}\right)\right) \epsilon_{\mu \beta \gamma \delta}, \\
& \operatorname{Tr}\left(\left[D^{\mu} U\right]_{-}\left(\left[G^{\alpha \beta}\right]_{+}\left[H^{\gamma \delta}\right]_{+}\left[D_{\alpha} U\right]_{-}-\left[D_{\alpha} U\right]_{-}\left[H^{\gamma \delta}\right]_{+}\left[G^{\alpha \beta}\right]_{+}\right)\right) \epsilon_{\mu \beta \gamma \delta}, \\
& \operatorname{Tr}\left(\left[D^{\mu} U\right]_{-}\left(\left[D_{\alpha} U\right]_{-}\left[H^{\alpha \beta}\right]_{+}\left[G^{\gamma \delta}\right]_{+}-\left[G^{\gamma \delta}\right]_{+}\left[H^{\alpha \beta}\right]_{+}\left[D_{\alpha} U\right]_{-}\right)\right) \epsilon_{\mu \beta \gamma \delta}, \\
& \operatorname{Tr}\left(\left[D^{\mu} U\right]_{-}\left(\left[H^{\alpha \beta}\right]_{+}\left[D_{\alpha} U\right]_{-}\left[G^{\gamma \delta}\right]_{+}-\left[G^{\gamma \delta}\right]_{+}\left[D_{\alpha} U\right]_{-}\left[H^{\alpha \beta}\right]_{+}\right)\right) \epsilon_{\mu \beta \gamma \delta}, \\
& \operatorname{Tr}\left(\left[D^{\mu} U\right]_{-}\left(\left[H^{\alpha \beta}\right]_{+}\left[G^{\gamma \delta}\right]_{+}\left[D_{\alpha} U\right]_{-}-\left[D_{\alpha} U\right]_{-}\left[G^{\gamma \delta}\right]_{+}\left[H^{\alpha \beta}\right]_{+}\right)\right) \epsilon_{\mu \beta \gamma \delta} .
\end{aligned}
$$

There are two sets of epsilon relations, as described in Sec. IIE7, among this group of terms. One consists of two relations among the set Eqs. (215), (217), and (220) and will be used to eliminate Eqs. (217) and (220). The other involves three relations among the set Eqs. (213), (214), (216), (218), (219), and (221) and will be used to eliminate Eqs. (218), (219), and (221).

From 2a)' we obtain

$\operatorname{Tr}\left(\left[D_{\mu} U\right]_{-}\left(\left[D^{\mu} G^{\alpha \beta}\right]_{+}\left[H_{\alpha \beta}\right]_{+}-\left[G_{\alpha \beta}\right]_{+}\left[D^{\mu} H^{\alpha \beta}\right]_{+}-\left[H_{\alpha \beta}\right]_{+}\left[D^{\mu} G^{\alpha \beta}\right]_{+}+\left[D^{\mu} H^{\alpha \beta}\right]_{+}\left[G_{\alpha \beta}\right]_{+}\right)\right)$,

$\operatorname{Tr}\left(\left[D_{\alpha} U\right]_{-}\left(\left[D^{\gamma} G^{\alpha \beta}\right]_{+}\left[H_{\gamma \beta}\right]_{+}-\left[G^{\alpha \beta}\right]_{+}\left[D^{\gamma} H_{\gamma \beta}\right]_{+}-\left[H_{\gamma \beta}\right]_{+}\left[D^{\gamma} G^{\alpha \beta}\right]_{+}+\left[D^{\gamma} H_{\gamma \beta}\right]_{+}\left[G^{\alpha \beta}\right]_{+}\right)\right)$,

$\operatorname{Tr}\left(\left[D^{\gamma} U\right]_{-}\left(\left[D_{\alpha} G^{\alpha \beta}\right]_{+}\left[H_{\gamma \beta}\right]_{+}-\left[G^{\alpha \beta}\right]_{+}\left[D_{\alpha} H_{\gamma \beta}\right]_{+}-\left[H_{\gamma \beta}\right]_{+}\left[D_{\alpha} G^{\alpha \beta}\right]_{+}+\left[D_{\alpha} H_{\gamma \beta}\right]_{+}\left[G^{\alpha \beta}\right]_{+}\right)\right)$

$$
\operatorname{Tr}\left(\left[D^{\mu} U\right]_{-}\left(\left[D^{\nu} H^{\alpha \beta}\right]_{+}\left[H_{\alpha}^{\gamma}\right]_{+}-\left[H^{\alpha \beta}\right]_{+}\left[D^{\nu} H_{\alpha}^{\gamma}\right]_{+}\right)\right) \epsilon_{\mu \nu \beta \gamma}
$$

$\operatorname{Tr}\left(\left[D^{\mu} U\right]_{-}\left(\left[D_{\alpha} H^{\alpha \beta}\right]_{+}\left[H^{\gamma \delta}\right]_{+}-\left[H^{\alpha \beta}\right]_{+}\left[D_{\alpha} H^{\gamma \delta}\right]_{+}-\left[D_{\alpha} H^{\gamma \delta}\right]_{+}\left[H^{\alpha \beta}\right]_{+}+\left[H^{\gamma \delta}\right]_{+}\left[D_{\alpha} H^{\alpha \beta}\right]_{+}\right)\right) \epsilon_{\mu \beta \gamma \delta}$

$\operatorname{Tr}\left(\left[D_{\alpha} U\right]_{-}\left(\left[D^{\mu} H^{\alpha \beta}\right]_{+}\left[H^{\gamma \delta}\right]_{+}-\left[H^{\alpha \beta}\right]_{+}\left[D^{\mu} H^{\gamma \delta}\right]_{+}-\left[D^{\mu} H^{\gamma \delta}\right]_{+}\left[H^{\alpha \beta}\right]_{+}+\left[H^{\gamma \delta}\right]_{+}\left[D^{\mu} H^{\alpha \beta}\right]_{+}\right)\right) \epsilon_{\mu \beta \gamma \delta}$ 


$$
\begin{gathered}
\operatorname{Tr}\left(\left[D^{\mu} U\right]_{-}\left(\left[D^{\nu} G^{\alpha \beta}\right]_{+}\left[G_{\alpha}^{\gamma}\right]_{+}-\left[G^{\alpha \beta}\right]_{+}\left[D^{\nu} G_{\alpha}^{\gamma}\right]_{+}\right)\right) \epsilon_{\mu \nu \beta \gamma} \\
\operatorname{Tr}\left(\left[D^{\mu} U\right]_{-}\left(\left[D_{\alpha} G^{\alpha \beta}\right]_{+}\left[G^{\gamma \delta}\right]_{+}-\left[G^{\alpha \beta}\right]_{+}\left[D_{\alpha} G^{\gamma \delta}\right]_{+}-\left[D_{\alpha} G^{\gamma \delta}\right]_{+}\left[G^{\alpha \beta}\right]_{+}+\left[G^{\gamma \delta}\right]_{+}\left[D_{\alpha} G^{\alpha \beta}\right]_{+}\right)\right) \epsilon_{\mu \beta \gamma \delta} \\
\operatorname{Tr}\left(\left[D_{\alpha} U\right]_{-}\left(\left[D^{\mu} G^{\alpha \beta}\right]_{+}\left[G^{\gamma \delta}\right]_{+}-\left[G^{\alpha \beta}\right]_{+}\left[D^{\mu} G^{\gamma \delta}\right]_{+}-\left[D^{\mu} G^{\gamma \delta}\right]_{+}\left[G^{\alpha \beta}\right]_{+}+\left[G^{\gamma \delta}\right]_{+}\left[D^{\mu} G^{\alpha \beta}\right]_{+}\right)\right) \epsilon_{\mu \beta \gamma \delta} .
\end{gathered}
$$

There are two sets in this group, Eqs. (225), (226), and (227) and Eqs. (228), (229), and (230), each related by a single epsilon relation which we use to eliminate Eqs. (227) and (230).

Finally from $2 b)^{\prime}$ we get

$$
\begin{gathered}
\operatorname{Tr}\left(\left[D_{\mu} D^{\mu} U\right]_{-}\left(\left[G^{\alpha \beta}\right]_{+}\left[H_{\alpha \beta}\right]_{+}-\left[H_{\alpha \beta}\right]_{+}\left[G^{\alpha \beta}\right]_{+}\right)\right), \\
\operatorname{Tr}\left(\left[D_{\alpha} D^{\gamma} U\right]_{-}\left(\left[G^{\alpha \beta}\right]_{+}\left[H_{\gamma \beta}\right]_{+}-\left[H_{\gamma \beta}\right]_{+}\left[G^{\alpha \beta}\right]_{+}\right)\right), \\
\operatorname{Tr}\left(\left[D_{\mu} D^{\mu} U\right]_{-}\left[H^{\alpha \beta}\right]_{+}\left[H^{\gamma \delta}\right]_{+}\right) \epsilon_{\alpha \beta \gamma \delta}, \\
\operatorname{Tr}\left(\left[D^{\mu} D_{\alpha} U\right]_{-}\left(\left[H^{\alpha \beta}\right]_{+}\left[H^{\gamma \delta}\right]_{+}+\left[H^{\gamma \delta}\right]_{+}\left[H^{\alpha \beta}\right]_{+}\right)\right) \epsilon_{\mu \beta \gamma \delta}, \\
\operatorname{Tr}\left(\left[D_{\mu} D^{\mu} U\right]_{-}\left[G^{\alpha \beta}\right]_{+}\left[G^{\gamma \delta}\right]_{+}\right) \epsilon_{\alpha \beta \gamma \delta}, \\
\operatorname{Tr}\left(\left[D^{\mu} D_{\alpha} U\right]_{-}\left(\left[G^{\alpha \beta}\right]_{+}\left[G^{\gamma \delta}\right]_{+}+\left[G^{\gamma \delta}\right]_{+}\left[G^{\alpha \beta}\right]_{+}\right)\right) \epsilon_{\mu \beta \gamma \delta} .
\end{gathered}
$$

There are two epsilon relations for this group, which allow us to eliminate Eq. (234) in favor of Eq. (233) and Eq. (236) in favor of Eq. (235). Note that this is again a case where it is important, if we are to minimize the number of terms, to use the relations to eliminate the non equation of motion terms, as the equation of motion terms can be eliminated in a different way.

\section{Terms with no $D_{\mu}$ 's and three $\chi^{\mu \nu}$ 's}

Consider now the final case with three $\chi^{\mu \nu}$ 's and no covariant derivatives. This is relatively simple compared with the previous cases and we can write the general case directly,

$$
\left(1+(-1)^{s+p_{1}+p_{2}+p_{3}+\epsilon}\right)\left[\chi_{1}^{\mu \nu}\right]_{ \pm}\left(\left[\chi_{2}^{\alpha \beta}\right]_{ \pm}\left[\chi_{3}^{\gamma \delta}\right]_{ \pm}+(-1)^{c_{1}+c_{2}+c_{3}}\left[\chi_{3}^{\gamma \delta}\right]_{ \pm}\left[\chi_{2}^{\alpha \beta}\right]_{ \pm}\right) .
$$


Here $p_{i}$ and $c_{i}$ are the intrinsic parity and charge conjugation quantum numbers of the three $\chi$ 's. The \pm signs are not correlated and as before $(-1)^{s}$ is the product of the signs coming from the parity transformation on the individual $[A]_{+}$'s, counting + for $[A]_{+}$and - for $[A]_{-}$. By virtue of the second term with factors in reverse order, which comes from charge conjugation, the interchange $\chi_{2}$ and $\chi_{3}$ gives, up to a sign, the original expression. Thus the three $\chi$ 's can be treated as distinguishable from the beginning.

For the simplest case with three simple $\chi$ 's all of the $p_{i}$ and $c_{i}$ as well as $\epsilon$ are zero, which requires $s=0$ also. Thus we get

$$
\begin{gathered}
\operatorname{Tr}\left([\chi]_{+}[\chi]_{+}[\chi]_{+}\right), \\
\operatorname{Tr}\left([\chi]_{-}[\chi]_{-}[\chi]_{+}\right), \\
\operatorname{Tr}\left([\chi]_{+}\right) \operatorname{Tr}\left([\chi]_{+}[\chi]_{+}\right), \\
\operatorname{Tr}\left([\chi]_{+}\right) \operatorname{Tr}\left([\chi]_{-}[\chi]_{-}\right), \\
\operatorname{Tr}\left([\chi]_{-}\right) \operatorname{Tr}\left([\chi]_{-}[\chi]_{+}\right), \\
\operatorname{Tr}\left([\chi]_{+}\right) \operatorname{Tr}\left([\chi]_{+}\right) \operatorname{Tr}\left([\chi]_{+}\right), \\
\operatorname{Tr}\left([\chi]_{+}\right) \operatorname{Tr}\left([\chi]_{-}\right) \operatorname{Tr}\left([\chi]_{-}\right) .
\end{gathered}
$$

There are no terms with two $\chi^{\prime}$ 's and one $\chi^{\mu \nu}$ as there is nothing with which to contract the indices. With only one simple $\chi$ the general form of Eq. (237) reduces to

$$
\left(1+(-1)^{s+p_{2}+p_{3}+\epsilon}\right)[\chi]_{ \pm}\left(\left[\chi_{2}^{\alpha \beta}\right]_{+}\left[\chi_{3}^{\gamma \delta}\right]_{+}+(-1)^{c_{2}+c_{3}}\left[\chi_{3}^{\gamma \delta}\right]_{+}\left[\chi_{2}^{\alpha \beta}\right]_{+}\right)
$$

since $\left[\chi^{\mu \nu}\right]_{-}=0$ for $\mu \neq \nu$. We see from this that if $\chi_{2}=\chi_{3}$, so that both are $H^{\mu \nu}$ 's or both are $G^{\mu \nu}$ 's, then $s=1$ if there is an epsilon term and $s=0$ if not. This is reversed if $\chi_{2} \neq \chi_{3}$. The number of multiple traces will be quite limited because $\operatorname{Tr}\left(\left[\chi^{\mu \nu}\right]_{ \pm}\right)=0$ when $\mu \neq \nu$. Thus we obtain

$$
\begin{gathered}
\operatorname{Tr}\left([\chi]_{+}\left[H^{\mu \nu}\right]_{+}\left[H_{\mu \nu}\right]_{+}\right) \\
\operatorname{Tr}\left([\chi]_{+}\left[G^{\mu \nu}\right]_{+}\left[G_{\mu \nu}\right]_{+}\right), \\
\operatorname{Tr}\left([\chi]_{-}\left(\left[H^{\mu \nu}\right]_{+}\left[G_{\mu \nu}\right]_{+}-\left[G_{\mu \nu}\right]_{+}\left[H^{\mu \nu}\right]_{+}\right)\right), \\
\operatorname{Tr}\left([\chi]_{+}\right) \operatorname{Tr}\left(\left[H^{\mu \nu}\right]_{+}\left[H_{\mu \nu}\right]_{+}\right),
\end{gathered}
$$




$$
\begin{gathered}
\operatorname{Tr}\left([\chi]_{+}\right) \operatorname{Tr}\left(\left[G^{\mu \nu}\right]_{+}\left[G_{\mu \nu}\right]_{+}\right) \\
\operatorname{Tr}\left([\chi]_{-}\left[H^{\alpha \beta}\right]_{+}\left[H^{\gamma \delta}\right]_{+}\right) \epsilon_{\alpha \beta \gamma \delta}, \\
\operatorname{Tr}\left([\chi]_{-}\left[G^{\alpha \beta}\right]_{+}\left[G^{\gamma \delta}\right]_{+}\right) \epsilon_{\alpha \beta \gamma \delta}, \\
\operatorname{Tr}\left([\chi]_{+}\left(\left[H^{\alpha \beta}\right]_{+}\left[G^{\gamma \delta}\right]_{+}\left[G^{\gamma \delta}\right]_{+}\left[H^{\alpha \beta}\right]_{+}\right)\right) \epsilon_{\alpha \beta \gamma \delta}, \\
\operatorname{Tr}\left([\chi]_{-}\right) \operatorname{Tr}\left(\left[H^{\alpha \beta}\right]_{+}\left[H^{\gamma \delta}\right]_{+}\right) \epsilon_{\alpha \beta \gamma \delta}, \\
\operatorname{Tr}\left([\chi]_{-}\right) \operatorname{Tr}\left(\left[G^{\alpha \beta}\right]_{+}\left[G^{\gamma \delta}\right]_{+}\right) \epsilon_{\alpha \beta \gamma \delta} .
\end{gathered}
$$

Finally when all three $\chi^{\prime}$ 's are $\chi^{\mu \nu}$ 's the number of terms is very limited because of the parity and charge conjugation factors and because all factors are traceless. We find

$$
\begin{gathered}
\operatorname{Tr}\left(\left[G^{\mu \nu}\right]_{+}\left[G_{\mu \alpha}\right]_{+}\left[G_{\nu}^{\alpha}\right]_{+}\right) \\
\operatorname{Tr}\left(\left[G^{\mu \nu}\right]_{+}\left[H_{\mu \alpha}\right]_{+}\left[H_{\nu}^{\alpha}\right]_{+}\right), \\
\operatorname{Tr}\left(\left[G^{\mu \nu}\right]_{+}\left(\left[G^{\alpha \beta}\right]_{+}\left[H_{\beta}^{\gamma}\right]_{+}-\left[H^{\alpha \beta}\right]_{+}\left[G_{\beta}^{\gamma}\right]_{+}\right)\right) \epsilon_{\mu \nu \alpha \gamma} .
\end{gathered}
$$

The last of these, Eq. (258), is identically zero by virtue of the epsilon relations of Sec. IIE7.

\section{SIMPLIFICATION AND REORGANIZATION OF TERMS IN THE LAGRANGIAN - FINAL RESULTS}

We have now derived in Secs. III A - IIID the complete set of terms contributing to the order $p^{6}$ Lagrangian $\mathcal{L}_{6}$. The results are scattered through these sections in the order they were derived. We now want to collect those results in one place in a form of a Lagrangian with effective coefficients analogous to the standard Gasser-Leutwyler Lagrangian. In the course of doing this we want to simplify the forms as much as possible and to reorganize them so as to select out those terms which are likely to be most immediately useful.

\section{A. Equation of motion terms}

In the course of the derivation we have extracted as many terms as possible which are proportional to the factor $\left[D_{\mu} D^{\mu} U\right]_{-}$which we have called the 'equation of motion terms' and have stated, but not proved, that these can be transformed away. Details of this transformation procedure are given with respect to $\mathcal{L}_{6}$ in [43] and with respect to lower 
orders in a number of earlier papers [14,16, 17]. Here we just outline the general idea, as it produces a large reduction in the final number of terms which need to be considered for the general Lagrangian.

The lowest order Lagrangian is given by

$$
\mathcal{L}_{2}=\frac{F_{0}^{2}}{4} \operatorname{Tr}\left(D_{\mu} U\left(D^{\mu} U\right)^{\dagger}\right)+\frac{F_{0}^{2}}{4} \operatorname{Tr}\left(\chi U^{\dagger}+U \chi^{\dagger}\right) .
$$

From this one can obtain the lowest order or classical equation of motion $\mathcal{O}_{E O M}^{(2)}=0$ where

$$
\mathcal{O}_{E O M}^{(2)}=2\left[D_{\mu} D^{\mu} U\right]_{-}-2[\chi]_{-}+\frac{2}{3} \operatorname{Tr}\left([\chi]_{-}\right) .
$$

We observe first that we can make the replacement $\left[D_{\mu} D^{\mu} U\right]_{-} \rightarrow \mathcal{O}_{E O M}^{(2)}$ in each term where it appears, where as always we have dropped the irrelevant numerical factor. Since we have the most general form the extra terms added and subtracted to get $\mathcal{O}_{E O M}^{(2)}$ are just terms we already have.

Now make a transformation on the fields of the form

$$
U \rightarrow U^{\prime}=\exp (i S) U
$$

where $S=S^{\dagger}$ and $\operatorname{Tr}(S)=0$. It is a general result 44 49 that such a transformation does not affect measurable quantities such as the S-matrix. Applied to $\mathcal{L}_{2}$ it generates a correction to lowest order in $S$ of the form [43]

$$
\delta \mathcal{L}_{2}=\frac{F_{0}^{2}}{4} \operatorname{Tr}\left(i S \mathcal{O}_{E O M}^{(2)}\right)
$$

and we can choose an $S$ of order $p^{2}$ so that this term cancels the equation of motion terms in $\mathcal{L}_{4}$. This transformation generates corrections at order $p^{6}$ as well [43], both from the second order in $S$ correction to $\mathcal{L}_{2}$ and the first order in $S$ correction to $\mathcal{L}_{4}$. For our purposes these corrections can simply be absorbed in the terms of $\mathcal{L}_{6}$ since we have the most general form. Finally we make a second transformation on $\mathcal{L}_{2}$ using an $S$ of order $p^{4}$ and thus generate a correction term analogous to that of Eq. (262) which is of order $p^{6}$ and proportional to $\mathcal{O}_{E O M}^{(2)}$. By choosing $S$ properly we can eliminate those terms of $\mathcal{L}_{6}$ which contain $\mathcal{O}_{\text {EOM }}^{(2)}$.

The conclusion one draws from this discussion is that in order to generate the most general $\mathcal{L}_{6}$ in its simplest form, we can just drop all terms proportional to $\mathcal{O}_{E O M}^{(2)}$. This allows us to eliminate the 23 terms given by Eqs. (41), (42), (45), (59), (60), (61), (62), (83), (84), (86), 89), (95), (96), (97), (98), (102), (118), (122), (137), (158), (159), (160), (231) and the additional 6 terms containing an $\epsilon_{\alpha \beta \gamma \delta}$ given by Eqs. (46), (111), (112), (125), (233), (235). For completeness, these terms are given explicitly in Table VIII in Appendix B. Recall that three additional equation of motion terms, Eqs. (43), (44), and (85), had previously been eliminated using the trace relations.

It is important to note that this argument must be applied with extreme care if one works in the other direction. Thus if one starts with a particular $\mathcal{L}_{6}$ generated from some model which contains terms proportional to $\mathcal{O}_{E O M}^{(2)}$ and tries to put it into the general form we have derived it will be necessary to keep track of the changes in the coefficients generated by the successive transformations, particularly the second order correction to $\mathcal{L}_{2}$ which would be missed by just dropping the $\mathcal{O}_{E O M}^{(2)}$ terms. This is explained in more detail in [43]. 


\section{B. Reorganization}

In the course of the derivations of the preceding sections we devoted a great deal of effort to getting all of the terms of the Lagrangian in a systematic way, without any thought at all as to which terms would be of most practical importance. It is clear however that there will be some terms which will be of immediate importance for simple processes. In fact there have been already recent calculations, e.g. Ref. [39], which in the absence of the general $\mathcal{L}_{6}$, have included a few ad hoc $p^{6}$ terms, motivated by the need to cancel infinities arising from the loops involving $\mathcal{L}_{4}$. On the other hand some terms in $\mathcal{L}_{6}$ contribute only to processes which are so complicated that they probably will not be of practical interest for a long time. For example, naively the factor $\left[D_{\mu} U\right]_{-}$goes like $\partial_{\mu} \phi$ in leading order and so it would seem that a term like that of Eq. (54) would contribute at tree level only to a process involving six boson fields, which is probably not of much immediate interest.

One concludes from the preceding discussion that it would be useful to organize the terms in the Lagrangian in such a way as to separate out those contributing to simple processes. Such an organization is more subtle than it might seem however. Consider for example the simplest factor appearing in many terms of the Lagrangian, $\left[D_{\mu} U\right]_{-}$. If we start with Eq. (15) for $U$ and expand in powers of $\phi$ we get symbolically $U \sim 1+i \phi+\mathcal{O}\left(\phi^{2}\right)$, where we have absorbed the $F_{0}$ into $\phi$ for the purposes of this section. Now using the first line of Eq. (18) we expand $\left[D_{\mu} U\right]_{-}$as

$$
\left[D_{\mu} U\right]_{-} \sim i \partial_{\mu} \phi-i\left(R_{\mu}-L_{\mu}\right)+\left[L_{\mu}, \phi\right]+\mathcal{O}\left(\phi^{2}\right) .
$$

If $R_{\mu}=L_{\mu}=0$, corresponding to pure QCD with no external fields, then $\left[D_{\mu} U\right]_{-} \sim \partial_{\mu} \phi$ and a term like Eq. (54) which involves six $D_{\mu}$ 's does contribute only to a process with six bosons in accordance with our naive expectation. In general however when $R_{\mu} \neq L_{\mu}$ this term could contribute to anything with $n$ bosons and $6-n$ external fields, where $0 \leq n \leq 6$. Thus for the general case it doesn't appear possible to sort the terms in a useful way.

However if we limit the external fields to the electromagnetic field, which is a useful physical situation, then we can take $R_{\mu}=L_{\mu}=-e A_{\mu} Q$, where $A_{\mu}$ is the electromagnetic field, $e>0$ is the electric charge, and $Q$ is the diagonal quark charge matrix $3 Q=\operatorname{diag}(2,-1,-1)$. This allows us to classify the various terms according to the number of boson and external electromagnetic fields required in the process in order that the term produce a non zero contribution in this limit. The explicit term of course remains perfectly general, but the sorting into various groups depends on this special assumption.

With the assumption of only external electromagnetic fields, i.e. $R_{\mu}=L_{\mu}=-e A_{\mu} Q$, and with $\chi=\chi^{\dagger}$ the various building blocks contribute to order $\phi$ and lower as:

$$
\begin{aligned}
{\left[D_{\mu} U\right]_{-} } & \sim \phi, A \phi, \\
{\left[D_{\mu} D_{\nu} U\right]_{-} } & \sim \phi, A \phi, A^{2} \phi, \\
{[\chi]_{+} } & \sim \chi, \chi \phi, \\
{\left[D_{\mu} \chi\right]_{+} } & \sim \chi, \chi \phi, A \chi, A \chi \phi, \\
{[\chi]_{-} } & \sim \chi \phi, \\
{\left[D_{\mu} \chi\right]_{-} } & \sim \chi \phi, A \chi \phi,
\end{aligned}
$$




$$
\begin{aligned}
{\left[D_{\mu} D_{\nu} \chi\right]_{-} } & \sim \chi \phi, A \chi \phi, A^{2} \chi \phi, \\
{\left[G^{\mu \nu}\right]_{+} } & \sim A, A \phi, \\
{\left[D_{\alpha} G^{\mu \nu}\right]_{+} } & \sim A, A \phi, \\
{\left[D_{\alpha} D_{\beta} G^{\mu \nu}\right]_{+} } & \sim A, A \phi, \\
{\left[H^{\mu \nu}\right]_{+} } & \sim A \phi, \\
{\left[D_{\alpha} H^{\mu \nu}\right]_{+} } & \sim A \phi, A^{2} \phi, \\
{\left[D_{\alpha} D_{\beta} H^{\mu \nu}\right]_{+} } & \sim A \phi, A^{2} \phi, A^{3} \phi .
\end{aligned}
$$

Note that for the electromagnetic case and in addition the usual choice of $\chi=2 B_{0} M$, with $M$ the diagonal quark mass matrix, $M=\operatorname{diag}\left(m_{u}, m_{d}, m_{s}\right)$, the quantities $\left[D_{\mu} \chi\right]_{ \pm}$and $\left[D_{\mu} D_{\nu} \chi\right]_{ \pm}$actually vanish since $[Q, \chi]=0$ implies $D_{\mu} \chi \rightarrow \partial_{\mu} \chi$. We keep them, however, to preserve a bit more generality.

There is another useful simplification we can use also. Under parity, using the results of Sec. IIE5, we have in effect for each term $U \rightarrow U^{\dagger}, \chi \rightarrow \chi^{\dagger}, R_{\mu} \leftrightarrow L^{\mu}$ and $F_{R}^{\mu \nu} \leftrightarrow F_{\mu \nu}^{L}$, with an extra minus sign for the $\epsilon_{\alpha \beta \gamma \delta}$ terms. This means that for the electromagnetic or pure QCD case, with $R_{\mu}=L_{\mu}$ and $F_{R}^{\mu \nu}=F_{L}^{\mu \nu}$, and with $\chi=\chi^{\dagger}$, we can use the fact that $U \rightarrow U^{\dagger}$ is equivalent to $\phi \rightarrow-\phi$ to show that parity invariance implies that terms without an $\epsilon_{\alpha \beta \gamma \delta}$ will have only even powers of $\phi$ while those with an $\epsilon_{\alpha \beta \gamma \delta}$ will have odd powers [29, 30.

We have used both of these simplifications to group the terms in the final result of Tables [11 VII according to the smallest number of $\phi$ 's and $A_{\mu}$ 's which a term can have. There can be more than the minimum number of course, if one goes past leading order in the expansion in powers of $\phi$. Also it is possible that there may be accidental cancellations which make the leading term vanish so that a given structure may in fact have more $\phi$ 's or $A$ 's than indicated in the tables. In a few cases the leading behavior of the structure contains no $\phi$ 's. These terms have been included with the terms having two $\phi$ 's, since such non leading contributions would seem most relevant for practical calculations.

To see how this sorting works, suppose we are interested in the contact terms at order $p^{6}$ contributing to the process $\gamma+\gamma \rightarrow \pi+\pi$. We thus need two $\phi$ 's and two $A_{\mu}$ 's in tree level. Thus none of the terms in Tables [II - VII contribute as they require an odd number or too many $\phi$ 's. Of the terms in Table II the first group has no $A_{\mu}$ 's and the last group has too many, so neither will contribute. Thus we need consider only the middle three groups, and even some of those terms will vanish because as noted above $\left[D_{\mu} \chi\right]_{ \pm}$and $\left[D_{\mu} D_{\nu} \chi\right]_{ \pm}$vanish.

If one wishes to consider a general external interaction which has $R_{\mu} \neq L_{\mu}$ then there seems to be no substitute for considering each term in detail to determine which will contribute. We emphasize again that the results listed in Tables [1] - VII are completely general and appropriate also for general external interactions. Only the classification into groups depends on the assumption of just the electromagnetic interaction.

\section{Simplifications}

The notation used so far was developed to simplify the derivations. It has the advantage of leading to a Lagrangian which depends on a relatively small number of building blocks 
which have well defined and simple transformation properties under parity and charge conjugation and hermitian conjugation.

For the purposes of calculation however there are a few simplifications which will be collected here for reference, though most have been mentioned earlier. Some may simplify the evaluations in specific cases.

The building block $\left[D_{\mu} U\right]_{-}$can be written as $\left[D_{\mu} U\right]_{-}=\left(D_{\mu} U\right) U^{\dagger}=-U\left(D_{\mu} U\right)^{\dagger}$. Under the trace the $U^{\prime}$ 's and $U^{\dagger}$ 's for the most part commute through and collapse to unity so that the effect is to convert a string of $\left[D_{\mu} U\right]_{-}$'s to a string of $\left(D_{\mu} U\right)^{\text {'s }}$ and $\left(D_{\mu} U\right)^{\dagger}$ 's.

The factors $\left[G^{\mu \nu}\right]_{+}$and $\left[H^{\mu \nu}\right]_{+}$can be expressed in terms of the original $F_{R}^{\mu \nu}$ and $F_{L}^{\mu \nu}$ via

$$
\begin{aligned}
& {\left[G^{\mu \nu}\right]_{+}=G^{\mu \nu} U^{\dagger}=U G^{\mu \nu \dagger}=\left(F_{R}^{\mu \nu}+U F_{L}^{\mu \nu} U^{\dagger}\right),} \\
& {\left[H^{\mu \nu}\right]_{+}=H^{\mu \nu} U^{\dagger}=U H^{\mu \nu \dagger}=\left(F_{R}^{\mu \nu}-U F_{L}^{\mu \nu} U^{\dagger}\right) .}
\end{aligned}
$$

For purely electromagnetic external gauge fields, with $A_{\mu}$ proportional to the (diagonal) quark charge matrix, or for pure QCD with no external gauge fields, and for the usual choice for $\chi$ as a diagonal quark mass matrix the covariant derivative $D_{\mu} \chi \rightarrow \partial_{\mu} \chi \rightarrow 0$. Thus in this situation the terms containing $\left[D_{\mu} \chi\right]_{ \pm}$or $\left[D_{\mu} D_{\nu} \chi\right]_{ \pm}$all vanish.

\section{Final Results}

We have collected our final results for $\mathcal{L}_{6}$, the complete Lagrangian to order $p^{6}$, in Tables II - VII, ordering the various terms according to the scheme described in Sec. IVB above. Each term has been written in such a way that it is chirally invariant and for real coefficients is hermitian and invariant under parity and charge conjugation. There are a total of 32 terms of odd intrinsic parity, corresponding to the coefficients $A_{i}$ and involving an $\epsilon_{\alpha \beta \gamma \delta}$. There are 111 terms of even intrinsic parity, corresponding to the coefficients $B_{i}$. In the course of the derivation we obtained 23 independent equation of motion terms proportional to the operator $\mathcal{O}_{E O M}^{(2)}$ of Eq. (260). For completeness these have been listed in Table VIII in Appendix B, though we assume that for the simplest Lagrangian these will have been transformed away via an appropriate field transformation [16, 43].

Also in the course of the derivation we used trace relations to express 18 structures originally obtained in terms of others. Table $\mathbb{E}$ of Appendix $\mathrm{A}$ shows which of the original terms are related and which we chose to eliminate. Likewise epsilon relations were used to eliminate 16 dependent structures from the original set of odd intrinsic parity terms. Table X of Appendix $\mathrm{Q}$ indicates which of the original equations were eliminated. Both of these tables, though not strictly necessary for the final results, should make it easier to compare our work with that of others.

We have tried to start with all possible structures and to eliminate those which are not independent and to extract from the remaining terms as many equation of motion terms as possible. The procedure for doing this requires several tricks, i.e. the trace and epsilon relations, and depends in some cases on the way in which one writes the various terms. We cannot prove in a general and rigorous way that the resulting terms are all independent. Thus the reader should be aware that it is possible that there may be additional tricks which have been missed which could be used to express some of the structures in terms of others and thus reduce the number of independent terms. 


\section{E. Comparison with other results}

The set of independent structures of $O\left(p^{6}\right)$ in the odd intrinsic parity sector has already been discussed by Issler [34 and by Akhoury and Alfakih 36. However, the number of independent terms we find does not agree with either of the above references, which mutually disagree with each other. In the following, we will try to locate potential sources of this discrepancy. A direct term-by-term comparison is made difficult by the fact that, in general, different conventions and, furthermore, different basic building blocks are used.

Let us start with Ref. [34] which quotes 49 independent terms. This number is close to our starting number of 54 terms. However, we made use of the epsilon relations to eliminate 16 terms. It appears that these relations were not used in any derivation prior to the work by Akhoury and Alfakih [36] (see for example [33]). Thus one has to conclude that, in general, too many terms were found which, in fact, are not independent. Furthermore, there is no reference in [34] to the use of the equation of motion or field transformations to eliminate terms. For example, of the 4 terms proportional to $k_{1}^{(0)}-k_{4}^{(0)}$ in Ref. [34], the epsilon relation can be used to eliminate say the structure proportional to $k_{4}^{(0)}$, and from the remaining 3 terms the ones proportional to $k_{1}^{(0)}$ and $k_{2}^{(0)}$ can be related using a field transformation, resulting in only two, instead of four, independent structures. We made use of 6 field transformations which reduces our final number to 32 as compared with 49 in Ref. 34.

However, even after taking these two observations into consideration, there remain some discrepancies. It appears that in Ref. [34] not all independent orderings of operators under the trace have been taken into account. As an example, in our opinion, there should be another term similar to the structure proportional to $k_{12}^{(1)}$ involving a different contraction of indices.

Finally, it appears that the set of terms includes structures which can be related using the total derivative argument resulting in a reduction of the number of terms. To be specific, let us consider as an example the structure proportional to $k_{17}^{(1)}$. It is straightforward but tedious to show that up to a total derivative it is related to the terms proportional to $k_{5}^{(1)}, k_{9}^{(1)}, k_{14}^{(1)}$ and $k_{11}^{(2)}$. For that purpose one has to take the covariant derivative off of the field strength tensor in the term proportional to $k_{17}^{(1)}$ and use the total derivative argument as outlined in Sec. IIE 1.

A comparison with the work of Akhoury and Alfakih [36] turns out to be more difficult as their choice of the building blocks is very different from ours. The final number quoted in Ref. [36] is 30 where 5 terms have been eliminated using the equation of motion. This has to be compared with our 32 terms using 6 equation of motion terms.

Even though the use of epsilon relations was first proposed in Ref. [36] it seems that their set still contains structures which are not independent as a consequence of such relations. To give an example, the terms proportional to $w_{11}-w_{13}$ can be interpreted to originate from a tensor $Q_{\alpha \beta \mu \nu \rho \sigma}$ which is antisymmetric in the index pairs $(\alpha, \beta),(\mu, \nu)$, and $(\rho, \sigma)$, respectively. Without the epsilon relation, one would naively expect 3 independent contractions from such a tensor, of which only one independent term remains after use of the epsilon relation. In a similar fashion one can show that of the four terms proportional to $w_{7}-w_{10}$ only three are independent. Finally, the very first structure proportional to $w_{1}$ 
vanishes identically (see our Eq. (258)).

On the other hand, it appears that there are terms missing in Ref. [36]. To be specific, there exists an additional independent contraction of indices for the structure of the type proportional to $w_{6}$. Furthermore, Ref. [36] does not contain any terms involving covariant derivatives of $\chi$ of which we find three independent terms. Finally, note that in Ref. [36] the equation of motion is used so as to eliminate terms which are proportional to $[\chi]_{-}$instead of the structures proportional to $\left[D_{\mu} D^{\mu} U\right]_{-}$. On the other hand this means that in Ref. [36] terms proportional to $\left[D_{\mu} D^{\mu} U\right]_{-}$should have been kept. In fact, we find six such terms (see Table (VIII) whereas Ref. [36] quotes only three.

Thus it appears that in both of the previous cases where a systematic study of the odd intrinsic parity terms was made there are terms in the resulting sets which are not independent and terms which have been missed.

\section{SUMMARY}

In the preceding sections we have developed the complete chirally invariant Lagrangian $\mathcal{L}_{6}$ for the meson sector to order $p^{6}$. This is intended to be an extension of the order $p^{4}$ Lagrangian $\mathcal{L}_{4}$ of Gasser and Leutwyler which has become the standard in chiral perturbation theory and has been used in many applications. Such an extension is important at this time because we are beginning to see two loop calculations of processes for which the leading contributions are order $p^{4}$. Such calculations generate some $p^{6}$ contributions, but the full $\mathcal{L}_{6}$ is needed to produce a consistent result.

Throughout we have emphasized a careful and pedagogical development of the steps leading to the full Lagrangian, since we feel that it is only via such an approach that the reader can be confident that the extremely complicated final result is complete and correct. To do this we have first outlined a hierarchical strategy which allows us to eliminate terms in favor of ones lower in the hierarchy. We then discussed a number of general results which allowed us to simplify and reduce the number of terms. After imposing parity and charge conjugation invariance we could obtain a set of general structures at each level, which could then be evaluated to find the set of possible terms. Trace relations and epsilon relations were then used to eliminate terms which were not independent and we described how field transformations could eliminate those terms proportional to the lowest order equation of motion.

The resulting set of terms was then sorted, for the usual QCD plus electromagnetic case, according to the minimum number of boson and electromagnetic fields appearing. The final result for $\mathcal{L}_{6}$ is given in Tables $\mathbb{M}$ - $\nabla T$. It consists of 111 terms in the even intrinsic parity sector and 32 terms in the odd intrinsic parity sector.

To our knowledge there have been no prior systematic studies of the even intrinsic parity sector to this order, though isolated terms have been used in a variety of calculations. In the odd sector however there have been two previous analyses [34, 36], which disagree in the number of terms with our result and with each other. We have shown that in each of these previous cases, terms have been missed and terms which are not independent have been included. 
It is clear that the coefficients of all of these terms will never be evaluated from experiment. However a much smaller subset actually contributes to most simple processes, and it may be possible to get information about some of them.

In any case we hope that our derivation of the complete and most general $\mathcal{L}_{6}$ Lagrangian will stimulate systematic chiral perturbation theory studies of processes at this order.

\section{ACKNOWLEDGMENTS}

This work was supported in part by a grant from the Natural Sciences and Engineering Research Council of Canada.

\section{APPENDIX A: RELATIONS BETWEEN TRACES}

Following the lecture notes of Coleman [50], we will derive relations between traces of $3 \times 3$ matrices. Let $A$ be any complex $3 \times 3$ matrix with eigenvalues $a_{1}, a_{2}$, and $a_{3}$ (possibly complex and identical). The solution of the characteristic equation is then equivalent to

$$
\left(A-a_{1} I\right)\left(A-a_{2} I\right)\left(A-a_{3} I\right)=0,
$$

where $I$ is the $3 \times 3$ identity matrix. As $A$ is similar to a matrix $B$ of the form [51,

$$
A=T B T^{-1}, \quad B=\left(\begin{array}{ccc}
a_{1} & \delta & 0 \\
0 & a_{2} & \delta^{\prime} \\
0 & 0 & a_{3}
\end{array}\right), \quad \delta, \delta^{\prime}=0 \text { or } 1,
$$

one finds

$$
\begin{aligned}
\operatorname{Tr}(A)=\operatorname{Tr}(B) & =a_{1}+a_{2}+a_{3}, \\
\operatorname{Tr}\left(A^{2}\right)=\operatorname{Tr}\left(B^{2}\right) & =a_{2}^{2}+a_{2}^{2}+a_{3}^{2}, \\
\operatorname{det}(A)=\operatorname{det}(B) & =a_{1} a_{2} a_{3} .
\end{aligned}
$$

These relations may be used to rewrite Eq. (A1) as

$$
A^{3}-\operatorname{Tr}(A) A^{2}+\frac{1}{2}\left((\operatorname{Tr}(A))^{2}-\operatorname{Tr}\left(A^{2}\right)\right) A-\operatorname{det}(A)=0 .
$$

A first important observation is made by taking the trace of Eq. (A4), namely that the determinant of a matrix can be expressed in terms of traces. This is the justification for not considering determinants as separate building blocks in the construction of the chiral Lagrangian [52]. Taking the trace of Eq. (A4) one eliminates the determinant to obtain (see also Eq. (80) of Ref. [53)

$$
\begin{aligned}
& A^{3}-\operatorname{Tr}(A) A^{2}+\frac{1}{2}(\operatorname{Tr}(A))^{2} A-\frac{1}{2} \operatorname{Tr}\left(A^{2}\right) A \\
& -\frac{1}{3} \operatorname{Tr}\left(A^{3}\right)+\frac{1}{2} \operatorname{Tr}\left(A^{2}\right) \operatorname{Tr}(A)-\frac{1}{6}(\operatorname{Tr}(A))^{3}=0 .
\end{aligned}
$$


Starting from Eq. A5 we will derive various trace relations for traces involving between four and six $3 \times 3$ matrices.

Multiplying Eq. (A5) by $A$ and taking the trace results in

$$
\operatorname{Tr}\left(A^{4}\right)-\frac{4}{3} \operatorname{Tr}\left(A^{3}\right) \operatorname{Tr}(A)-\frac{1}{2}\left(\operatorname{Tr}\left(A^{2}\right)\right)^{2}+\operatorname{Tr}\left(A^{2}\right)(\operatorname{Tr}(A))^{2}-\frac{1}{6}(\operatorname{Tr}(A))^{4}=0 .
$$

Inserting $A=\lambda_{1} A_{1}+\lambda_{2} A_{2}+\lambda_{3} A_{3}+\lambda_{4} A_{4}$ into Eq. (A6) and comparing the coefficients of $\lambda_{1} \lambda_{2} \lambda_{3} \lambda_{4}$ one finds (see also Eq. (81) of Ref. [53])

$$
\begin{aligned}
\sum_{6 \text { perm. }} \operatorname{Tr} & \left(A_{1} A_{2} A_{3} A_{4}\right)-\sum_{8 \text { perm. }} \operatorname{Tr}\left(A_{1} A_{2} A_{3}\right) \operatorname{Tr}\left(A_{4}\right)-\sum_{3 \text { perm. }} \operatorname{Tr}\left(A_{1} A_{2}\right) \operatorname{Tr}\left(A_{3} A_{4}\right) \\
& +\sum_{6 \text { perm. }} \operatorname{Tr}\left(A_{1} A_{2}\right) \operatorname{Tr}\left(A_{3}\right) \operatorname{Tr}\left(A_{4}\right)-\operatorname{Tr}\left(A_{1}\right) \operatorname{Tr}\left(A_{2}\right) \operatorname{Tr}\left(A_{3}\right) \operatorname{Tr}\left(A_{4}\right)=0 .
\end{aligned}
$$

In the following, we list special cases of Eq. (A7) which we used to relate different terms and thus eliminate redundant structures in the chiral Lagrangian:

$$
\begin{aligned}
\sum_{6 \text { perm. }} \operatorname{Tr}\left(A_{1} A_{2} A_{3} A_{4}\right)-\operatorname{Tr} & \left(A_{1} A_{2} A_{3}+A_{1} A_{3} A_{2}\right) \operatorname{Tr}\left(A_{4}\right) \\
& -\sum_{3 \text { perm. }} \operatorname{Tr}\left(A_{1} A_{2}\right) \operatorname{Tr}\left(A_{3} A_{4}\right)=0,
\end{aligned}
$$

for $\operatorname{Tr}\left(A_{1}\right)=\operatorname{Tr}\left(A_{2}\right)=\operatorname{Tr}\left(A_{3}\right)=0$, and $A_{4}$ arbitrary;

$$
\sum_{6 \text { perm. }} \operatorname{Tr}\left(A_{1} A_{2} A_{3} A_{4}\right)-\sum_{3 \text { perm. }} \operatorname{Tr}\left(A_{1} A_{2}\right) \operatorname{Tr}\left(A_{3} A_{4}\right)=0,
$$

for $\operatorname{Tr}\left(A_{i}\right)=0$;

$$
\begin{aligned}
2 \operatorname{Tr}\left(\left(A^{2} B\right.\right. & \left.\left.+A B A+B A^{2}\right) C\right)-2 \operatorname{Tr}\left(A^{2} B\right) \operatorname{Tr}(C) \\
& -\operatorname{Tr}\left(A^{2}\right) \operatorname{Tr}(B C)-2 \operatorname{Tr}(A B) \operatorname{Tr}(A C)=0,
\end{aligned}
$$

for $\operatorname{Tr}(A)=\operatorname{Tr}(B)=0$, and $C$ arbitrary;

$$
2 \operatorname{Tr}\left(\left(A^{2} B+A B A+B A^{2}\right) C\right)-\operatorname{Tr}\left(A^{2}\right) \operatorname{Tr}(B C)-2 \operatorname{Tr}(A B) \operatorname{Tr}(A C)=0,
$$

for $\operatorname{Tr}(A)=\operatorname{Tr}(B)=\operatorname{Tr}(C)=0$;

$$
\begin{gathered}
4 \operatorname{Tr}\left(A^{2} B^{2}\right)+2 \operatorname{Tr}(A B A B)-4 \operatorname{Tr}\left(A^{2} B\right) \operatorname{Tr}(B) \\
-\operatorname{Tr}\left(A^{2}\right) \operatorname{Tr}\left(B^{2}\right)-2(\operatorname{Tr}(A B))^{2}+\operatorname{Tr}\left(A^{2}\right)(\operatorname{Tr}(B))^{2}=0,
\end{gathered}
$$

for $\operatorname{Tr}(A)=0$, and $B$ arbitrary, and

$$
4 \operatorname{Tr}\left(A^{2} B^{2}\right)+2 \operatorname{Tr}(A B A B)-\operatorname{Tr}\left(A^{2}\right) \operatorname{Tr}\left(B^{2}\right)-2(\operatorname{Tr}(A B))^{2}=0,
$$

for $\operatorname{Tr}(A)=\operatorname{Tr}(B)=0$. 
The last relation, Eq. (A13), was already used by Gasser and Leutwyler in the construction of the $p^{4}$ Lagrangian [14. Note, however, that the matrices $A$ and $B$ do not have to be hermitian for Eq. (A13) to hold, as is sometimes stated in the literature. Furthermore, Eq. (A9) is the result obtained in Ref. [50].

Next we multiply Eq. (A5) by $A^{2}$, take the trace, and rewrite $\operatorname{Tr}\left(A^{4}\right)$ using Eq. (A6) to obtain

$$
\operatorname{Tr}\left(A^{5}\right)-\frac{5}{6} \operatorname{Tr}\left(A^{3}\right) \operatorname{Tr}\left(A^{2}\right)-\frac{5}{6} \operatorname{Tr}\left(A^{3}\right)(\operatorname{Tr}(A))^{2}+\frac{5}{6} \operatorname{Tr}\left(A^{2}\right)(\operatorname{Tr}(A))^{3}-\frac{1}{6}(\operatorname{Tr}(A))^{5}=0 .
$$

Inserting $A=\sum_{i=1}^{5} \lambda_{i} A_{i}$ into Eq. (A14) one finds

$$
\begin{array}{r}
\sum_{24 \text { perm. }} \operatorname{Tr}\left(A_{1} A_{2} A_{3} A_{4} A_{5}\right)-\sum_{20 \text { perm. }} \operatorname{Tr}\left(A_{1} A_{2} A_{3}\right) \operatorname{Tr}\left(A_{4} A_{5}\right) \\
-\sum_{20 \text { perm. }} \operatorname{Tr}\left(A_{1} A_{2} A_{3}\right) \operatorname{Tr}\left(A_{4}\right) \operatorname{Tr}\left(A_{5}\right)+2 \sum_{10 \text { perm. }} \operatorname{Tr}\left(A_{1} A_{2}\right) \operatorname{Tr}\left(A_{3}\right) \operatorname{Tr}\left(A_{4}\right) \operatorname{Tr}\left(A_{5}\right) \\
-4 \operatorname{Tr}\left(A_{1}\right) \operatorname{Tr}\left(A_{2}\right) \operatorname{Tr}\left(A_{3}\right) \operatorname{Tr}\left(A_{4}\right) \operatorname{Tr}\left(A_{5}\right)=0 .
\end{array}
$$

We applied the following special case of Eq. (A15)

$$
\begin{aligned}
\sum_{6 \text { perm }} \operatorname{Tr}(A A B B C)- & \frac{1}{2} \operatorname{Tr}\left(A^{2} C\right) \operatorname{Tr}\left(B^{2}\right)-\frac{1}{2} \operatorname{Tr}\left(B^{2} C\right) \operatorname{Tr}\left(A^{2}\right) \\
- & \operatorname{Tr}(A B C) \operatorname{Tr}(A B)-\operatorname{Tr}(A C B) \operatorname{Tr}(A B) \\
& -\operatorname{Tr}\left(A^{2} B\right) \operatorname{Tr}(B C)-\operatorname{Tr}\left(A B^{2}\right) \operatorname{Tr}(A C)=0,
\end{aligned}
$$

for $\operatorname{Tr}(A)=\operatorname{Tr}(B)=0$, and $C$ arbitrary.

For our final application we can restrict ourselves to $\operatorname{Tr}(A)=0$. Multiplying Eq. A5) by $A^{3}$, and taking the trace one obtains

$$
\operatorname{Tr}\left(A^{6}\right)-\frac{1}{2} \operatorname{Tr}\left(A^{4}\right) \operatorname{Tr}\left(A^{2}\right)-\frac{1}{3}\left(\operatorname{Tr}\left(A^{3}\right)\right)^{2}=0,
$$

for $\operatorname{Tr}(A)=0$. Inserting $A=\sum_{i=1}^{6} \lambda_{i} A_{i}$ with $\operatorname{Tr}\left(A_{i}\right)=0$ into Eq. (A17) yields

$$
\begin{aligned}
\sum_{120 \text { perm. }} \operatorname{Tr}\left(A_{1} A_{2} A_{3} A_{4} A_{5} A_{6}\right)- & \frac{2}{3} \sum_{90 \text { perm. }} \operatorname{Tr}\left(A_{1} A_{2} A_{3} A_{4}\right) \operatorname{Tr}\left(A_{5} A_{6}\right) \\
& -\sum_{40 \text { perm. }} \operatorname{Tr}\left(A_{1} A_{2} A_{3}\right) \operatorname{Tr}\left(A_{4} A_{5} A_{6}\right)=0,
\end{aligned}
$$

for $\operatorname{Tr}\left(A_{i}\right)=0$. As a special application we insert $A_{1}=P_{\alpha}, A_{2}=P^{\alpha}, A_{3}=P_{\beta}, A_{4}=P^{\beta}$, $A_{5}=P_{\gamma}$, and $A_{6}=P^{\gamma}$ into Eq. (A18) to obtain

$$
\begin{array}{r}
2 \operatorname{Tr}\left((P \cdot P)^{3}\right)+3 \operatorname{Tr}\left(P \cdot P P_{\mu} P \cdot P P^{\mu}\right)+6 \operatorname{Tr}\left(P \cdot P P_{\mu} P_{\nu} P^{\mu} P^{\nu}\right) \\
+3 \operatorname{Tr}\left(P_{\mu} P_{\nu} P^{\mu} P_{\rho} P^{\nu} P^{\rho}\right)+\operatorname{Tr}\left(P_{\mu} P_{\nu} P_{\rho} P^{\mu} P^{\nu} P^{\rho}\right) \\
-\operatorname{Tr}(P \cdot P) \operatorname{Tr}\left((P \cdot P)^{2}\right)-\frac{1}{2} \operatorname{Tr}(P \cdot P) \operatorname{Tr}\left(P_{\mu} P_{\nu} P^{\mu} P^{\nu}\right) \\
-4 \operatorname{Tr}\left(P_{\mu} P_{\nu}\right) \operatorname{Tr}\left(P \cdot P P^{\mu} P^{\nu}\right)-2 \operatorname{Tr}\left(P_{\mu} P_{\nu}\right) \operatorname{Tr}\left(P^{\mu} P_{\rho} P^{\nu} P^{\rho}\right) \\
-3 \operatorname{Tr}\left(P \cdot P P_{\mu}\right) \operatorname{Tr}\left(P \cdot P P^{\mu}\right)-\operatorname{Tr}\left(P_{\mu} P_{\nu} P_{\rho}\right) \operatorname{Tr}\left(P^{\mu} P^{\nu} P^{\rho}\right) \\
-\operatorname{Tr}\left(P_{\mu} P_{\nu} P_{\rho}\right) \operatorname{Tr}\left(P^{\mu} P^{\rho} P^{\nu}\right)=0
\end{array}
$$


for $\operatorname{Tr}\left(P_{\alpha}\right)=0$.

In Table $[\mathrm{IX}$ we summarize how we applied the trace relations. It contains the equation numbers of structures which are not independent due to trace relations as well as the specific

trace relation which connects them. Furthermore, we list which structure we have chosen to eliminate.

\section{APPENDIX B: EQUATION OF MOTION TERMS}

For the purposes of completeness we list in Table VIII in the same form as our final results all of the terms in the original Lagrangian which are proportional to the factor $\left[D_{\mu} D^{\mu} U\right]_{-}$, which has been replaced by $\mathcal{O}_{E O M}^{(2)}$. As detailed in Sec. IVA these terms can simply be dropped from the most general form.

\section{APPENDIX C: EPSILON RELATIONS}

We list in Table $\mathrm{X}$ the equation numbers of the original structures we derived which are related by the epsilon relations of Sec. [IE7 and the ones which we chose to eliminate. 


\section{REFERENCES}

[1] S. L. Adler and R. F. Dashen, Current Algebras and Applications to Particle Physics, (Benjamin, New York, 1968).

[2] Sam B. Treiman, Roman Jackiw, David J. Gross, Lectures on Current Algebra and Its Applications, (Princeton University Press, Princeton, 1972).

[3] Ta-Pei Cheng and Ling-Fong Li, Gauge theory of elementary particle physics, (Oxford University Press, Oxford, 1984).

[4] Steven Weinberg, Phys. Rev. Lett. 18, 188 (1967).

[5] Steven Weinberg, Phys. Rev. 166, 1568 (1968).

[6] S. Gasiorowicz and D. A. Geffen, Rev. Mod. Phys. 41, 531 (1969).

[7] Roger Dashen and Marvin Weinstein, Phys. Rev. 183, 1261 (1969).

[8] William Marciano and Heinz Pagels, Phys. Rep. 36, 137 (1978).

[9] Guido Altarelli, Phys. Rep. 81, 1 (1982).

[10] Heinz Pagels, Phys. Rep. 16, 219 (1975).

[11] J. Gasser and H. Leutwyler, Phys. Rep. 87, 77 (1982).

[12] Steven Weinberg, Physica 96A, 327 (1979).

[13] J. Gasser and H. Leutwyler, Ann. Phys. 158, 142 (1984).

[14] J. Gasser and H. Leutwyler, Nucl. Phys. B250, 465 (1985).

[15] J. Gasser, QCD at low energies, Lecture notes "Troisieme Cycle de la Physique" (1988), unpublished.

[16] H. Leutwyler, in Perspectives in the Standard Model, Proceedings of the 1991 Theoretical Advanced Study Institute in Elementary Particle Physics, Boulder, Colorado, 2 - 28 June 1991, ed. by R. K. Ellis, C. T. Hill and J. D. Lykken (World Scientific, Singapore, 1992).

[17] John F. Donoghue, Eugene Golowich, Barry R. Holstein, Dynamics of the Standard Model (Cambridge University Press, Cambridge, 1992).

[18] Ulf-G. Meißner, Rept. Prog. Phys. 56, 903 (1993).

[19] A. Pich, Introduction to Chiral Perturbation Theory, Lectures given at the V Mexican School of Particles and Fields, Guanajuato, México, December 1992, preprint CERNTH.6978/93 (1993), hep-ph/9308351.

[20] G. Ecker, The Standard Model at Low Energies, Lectures given at the $6^{\text {th }}$ Indian Summer School on Intermediate Energy Physics Interaction in Hadronic Systems, Prague, August 25 - 31, 1993, to appear in the Proceedings (Czech. J. Phys.), preprint UWTHPH-199331 (1993), hep-ph/9309268.

[21] H. Leutwyler, Principles of Chiral Perturbation Theory, Lectures given at the Workshop "Hadrons 1994", Gramado, RS, Brasil, preprint BUTP-94/13 (1994), hep-ph/9406283.

[22] J. Bijnens, G. Colangelo and J. Gasser, preprint BUTP-94/4 (1994), hep-ph/9403390.

[23] János Balog, Phys. Lett. B149, 197 (1984).

[24] D. Ebert and H. Reinhardt, Nucl. Phys. B271, 188 (1986).

[25] G. Ecker, J. Gasser, A. Pich, E. de Rafael, Nucl. Phys. B321 311 (1989).

[26] John F. Donoghue, Carlos Ramirez, and German Valencia, Phys. Rev. D39, 1947 (1989).

[27] D. Espriu, E. de Rafael, J. Taron, Nucl. Phys. B345, 22 (1990).

[28] D. Ebert, A. A. Bel'kov, A. V. Lanyov, A. Schaale, Int. J. Mod. Phys. A8, 1313 (1993).

[29] Edward Witten, Nucl. Phys. B223, 422 (1983).

[30] Johan Bijnens, Int. J. Mod. Phys. A8, 3045 (1993). 
[31] J. Wess and B. Zumino, Phys. Lett. B37, 95 (1971).

[32] John F. Donoghue, Barry R. Holstein, and Y.-C. R. Lin, Phys. Rev. Lett. 55, 2766 (1985).

[33] John F. Donoghue and Daniel Wyler, Nucl. Phys. B316, 289 (1989).

[34] Dieter Issler, preprint SLAC-PUB-4943 (1989), unpublished.

[35] J. Bijnens, A. Bramon, F. Cornet, Z. Phys. C46, 599 (1990).

[36] R. Akhoury and A. Alfakih, Ann. Phys. 210, 81 (1991).

[37] Ll. Ametller, J. Bijnens, A. Bramon and F. Cornet, Phys. Lett. B276, 185 (1992).

[38] John F. Donoghue and Barry R. Holstein, Phys. Rev. D48, 137 (1993).

[39] S. Bellucci, J. Gasser and M. E. Sainio, preprint BUTP-93/18 (1994), hep-ph/9401206.

[40] A. A. Bel'kov, A. V. Lanyov, A. Schaale, and S. Scherer, preprint TRI-PP-94-69 (1994).

[41] M. Knecht, B. Moussallam, J. Stern, preprint IPNO/TH 94-08 (1994), hep-ph/9402318.

[42] J. D. Bjorken and S. D. Drell, Relativistic Quantum Fields (McGraw-Hill, New York, 1964).

[43] S. Scherer and H. W. Fearing, preprint TRI-PP-94-64 (1994), hep-ph/9408298.

[44] R. Haag, Phys. Rev. 112, 669 (1958).

[45] S. Kamefuchi, L. O’Raifeartaigh, and Abdus Salam, Nucl. Phys. 28, 529 (1961).

[46] S. Coleman, J. Wess and Bruno Zumino, Phys. Rev. 177, 2239 (1969).

[47] Howard Georgi, Nucl. Phys. B361, 339 (1991).

[48] Christopher Arzt, preprint UM-TH-92-28 (1992), hep-ph/9304230.

[49] Carsten Grosse-Knetter, Phys. Rev. D49, 6709 (1994).

[50] Sidney Coleman, Aspects of symmetry, (Cambridge University Press, Cambridge, 1985).

[51] Daniel T. Finkbeiner, II, Introduction to Matrices and Linear Transformations, (W. H. Freeman and Company, San Francisco, 1960).

[52] R. Sekhar Chivukula, Jonathan M. Flynn and Howard Georgi, Phys. Lett. B171, 453 (1986).

[53] Res Urech, preprint BUTP-94/9 (1994), hep-ph/9405341. 


\section{TABLES}

TABLE I. Transformation properties under the group $(\mathrm{G})$, charge conjugation $(\mathrm{C})$ and parity (P). The expressions for adjoint matrices are trivially obtained by taking the hermitian conjugate of each entry. In the parity transformed expression it is understood that the argument is $(-\vec{x}, t)$ and that partial derivatives $\partial_{\mu}$ act with respect to $x$ and not with respect to the argument of the corresponding function.

\begin{tabular}{cccc}
\hline \hline operator & $\mathrm{G}$ & $\mathrm{C}$ & $\mathrm{P}$ \\
\hline$U$ & $V_{R} U V_{L}^{\dagger}$ & $U^{T}$ & $U^{\dagger}$ \\
$D_{\lambda_{1}} \ldots D_{\lambda_{n}} U$ & $V_{R} D_{\lambda_{1}} \ldots D_{\lambda_{n}} U V_{L}^{\dagger}$ & $\left(D_{\lambda_{1}} \ldots D_{\lambda_{n}} U\right)^{T}$ & $\left(D^{\lambda_{1}} \ldots D^{\lambda_{n}} U\right)^{\dagger}$ \\
$\chi$ & $V_{R} \chi V_{L}^{\dagger}$ & $\chi^{T}$ & $\chi^{\dagger}$ \\
$D_{\lambda_{1}} \ldots D_{\lambda_{n}} \chi$ & $V_{R} D_{\lambda_{1}} \ldots D_{\lambda_{n}} \chi V_{L}^{\dagger}$ & $\left(D_{\lambda_{1}} \ldots D_{\lambda_{n}} \chi\right)^{T}$ & $\left(D^{\lambda_{1}} \ldots D^{\lambda_{n}} \chi\right)^{\dagger}$ \\
$R_{\mu}$ & $V_{R} R_{\mu} V_{R}^{\dagger}+i V_{R} \partial_{\mu} V_{R}^{\dagger}$ & $-L_{\mu}^{T}$ & $L^{\mu}$ \\
$L_{\mu}$ & $V_{L} L_{\mu} V_{L}^{\dagger}+i V_{L} \partial_{\mu} V_{L}^{\dagger}$ & $-R_{\mu}^{T}$ & $R^{\mu}$ \\
$F_{\mu \nu}^{R}$ & $V_{R} F_{\mu \nu}^{R} V_{R}^{\dagger}$ & $-\left(F_{\mu \nu}^{L}\right)^{T}$ & $F_{L}^{\mu \nu}$ \\
$F_{\mu \nu}^{L}$ & $V_{L} F_{\mu \nu}^{L} V_{L}^{\dagger}$ & $-\left(F_{\mu \nu}^{R}\right)^{T}$ & $F_{R}^{\mu \nu}$ \\
$G_{\mu \nu}$ & $V_{R} G_{\mu \nu} V_{L}^{\dagger}$ & $-G_{\mu \nu}^{T}$ & $G^{\mu \nu \dagger}$ \\
$D_{\lambda_{1}} \ldots D_{\lambda_{n}} G_{\mu \nu}$ & $V_{R} D_{\lambda_{1}} \ldots D_{\lambda_{n}} G_{\mu \nu} V_{L}^{\dagger}$ & $-\left(D_{\lambda_{1}} \ldots D_{\lambda_{n}} G_{\mu \nu}\right)^{T}$ & $\left(D^{\lambda_{1}} \ldots D^{\lambda_{n}} G^{\mu \nu}\right)^{\dagger}$ \\
$H_{\mu \nu}$ & $V_{R} H_{\mu \nu} V_{L}^{\dagger}$ & $H_{\mu \nu}{ }^{\dagger}$ & $-H^{\mu \nu \dagger}$ \\
$D_{\lambda_{1}} \ldots D_{\lambda_{n}} H_{\mu \nu}$ & $V_{R} D_{\lambda_{1}} \ldots D_{\lambda_{n}} H_{\mu \nu} V_{L}^{\dagger}$ & $\left(D_{\lambda_{1}} \ldots D_{\lambda_{n}} H_{\mu \nu}\right)^{T}$ & $-\left(D^{\lambda_{1}} \ldots D^{\lambda_{n}} H^{\mu \nu}\right)^{\dagger}$ \\
\hline \hline
\end{tabular}

TABLE II. Terms in the Lagrangian with 2 or more $\phi$ 's, sorted according to the minimum number of electromagnetic fields $A_{\mu}$. The formulas are general, but the sorting order and number of $\phi$ 's depends on the specific assumption of external electromagnetic fields only, i.e. on the assumption $R_{\mu}=L_{\mu}=-e A_{\mu} Q$, and on $\chi=\chi^{\dagger}$ as detailed in Sec. IVB. The quantities $\left[D_{\mu} \chi\right]_{ \pm}$and $\left[D_{\mu} D_{\nu} \chi\right]_{ \pm}$ vanish if one in addition makes the usual choice $\chi=2 B_{0} M$, but have been kept here for generality. Terms with leading behavior independent of $\phi$ have been included with the two $\phi$ terms. The double covariant derivative $D_{\mu} D_{\nu} A$ is assumed to be symmetric in its indices in accord with Eq. (27).

Terms in the Lagrangian with 2 or more $\phi$ 's and no $A_{\mu}$ 's possible

$+B_{2} \operatorname{Tr}([\chi]-[\chi]-[\chi]+)$

$+B_{3} \operatorname{Tr}\left([\chi]_{+}\right) \operatorname{Tr}\left([\chi]_{+}[\chi]_{+}\right)$

$+B_{4} \operatorname{Tr}\left([\chi]_{+}\right) \operatorname{Tr}\left([\chi]_{-}[\chi]_{-}\right)$

$+B_{5} \operatorname{Tr}\left([\chi]_{-}\right) \operatorname{Tr}\left([\chi]_{-}[\chi]_{+}\right)$

$+B_{6} \operatorname{Tr}\left([\chi]_{+}\right) \operatorname{Tr}\left([\chi]_{+}\right) \operatorname{Tr}\left([\chi]_{+}\right)$

$+B_{7} \operatorname{Tr}\left([\chi]_{+}\right) \operatorname{Tr}\left([\chi]_{-}\right) \operatorname{Tr}\left([\chi]_{-}\right)$ 
Terms in the Lagrangian with 2 or more $\phi^{\prime}$ 's, with an $A_{\mu}$ possible but not necessary

$+B_{9} \operatorname{Tr}\left(\left[D_{\alpha} D_{\beta} U\right]_{-}\left[D^{\alpha} U\right]_{-}\right) \operatorname{Tr}\left(\left[D^{\beta} \chi\right]_{+}\right)$

$+B_{10} \operatorname{Tr}\left(\left[D_{\mu} \chi\right]_{+}\left[D^{\mu} \chi\right]_{+}\right)$

$+B_{11} \operatorname{Tr}\left(\left[D_{\mu} \chi\right]_{-}\left[D^{\mu} \chi\right]_{-}\right)$

$+B_{12} \operatorname{Tr}\left(\left[D_{\mu} \chi\right]_{+}\right) \operatorname{Tr}\left(\left[D^{\mu} \chi\right]_{+}\right)$

$+B_{13} \operatorname{Tr}\left(\left[D_{\mu} \chi\right]_{-}\right) \operatorname{Tr}\left(\left[D^{\mu} \chi\right]_{-}\right)$

$+B_{14} \operatorname{Tr}\left(\left[D_{\mu} U\right]_{-}\left(\left[D^{\mu} \chi\right]_{+}[\chi]_{-}-[\chi]_{+}\left[D^{\mu} \chi\right]_{-}+[\chi]_{-}\left[D^{\mu} \chi\right]_{+}-\left[D^{\mu} \chi\right]_{-}[\chi]_{+}\right)\right)$

$+B_{15} \operatorname{Tr}\left(\left[D_{\mu} U\right]_{-}\left[D^{\mu} \chi\right]_{+}\right) \operatorname{Tr}\left([\chi]_{-}\right)-\operatorname{Tr}\left(\left[D_{\mu} U\right]_{-}[\chi]_{+}\right) \operatorname{Tr}\left(\left[D^{\mu} \chi\right]_{-}\right)$

$+B_{16} \operatorname{Tr}\left(\left[D_{\mu} U\right]_{-}\left[D^{\mu} \chi\right]_{-}\right) \operatorname{Tr}\left([\chi]_{+}\right)-\operatorname{Tr}\left(\left[D_{\mu} U\right]_{-}[\chi]_{-}\right) \operatorname{Tr}\left(\left[D^{\mu} \chi\right]_{+}\right)$

$+B_{17} \operatorname{Tr}\left(\left[D_{\mu} U\right]_{-}\left[D^{\mu} U\right]_{-}[\chi]_{+}[\chi]_{+}\right)$

$+B_{18} \operatorname{Tr}\left(\left[D_{\mu} U\right]_{-}\left[D^{\mu} U\right]_{-}[\chi]_{+}\right) \operatorname{Tr}\left([\chi]_{+}\right)$

$+B_{19} \operatorname{Tr}\left(\left[D_{\mu} U\right]_{-}\left[D^{\mu} U\right]_{-}\right) \operatorname{Tr}\left([\chi]_{+}[\chi]_{+}\right)$

$+B_{20} \operatorname{Tr}\left(\left[D_{\mu} U\right]_{-}[\chi]_{+}\right) \operatorname{Tr}\left(\left[D^{\mu} U\right]_{-}[\chi]_{+}\right)$

$+B_{21} \operatorname{Tr}\left(\left[D_{\mu} U\right]_{-}\left[D^{\mu} U\right]_{-}\right) \operatorname{Tr}\left([\chi]_{+}\right) \operatorname{Tr}\left([\chi]_{+}\right)$

\begin{tabular}{lr}
\hline \hline Terms in the Lagrangian with 2 or more $\phi^{\prime}$ s and at least $1 A_{\mu}$ & Eq. \\
\hline$+i B_{22} \operatorname{Tr}\left(\left[D_{\mu} D^{\beta} U\right]_{-}\left(\left[D_{\beta} U\right]_{-}\left[D_{\nu} G^{\mu \nu}\right]_{+}-\left[D_{\nu} G^{\mu \nu}\right]_{+}\left[D_{\beta} U\right]_{-}\right)\right)$ & 123 \\
$+i B_{23} \operatorname{Tr}\left(\left[D_{\mu} D^{\beta} U\right]_{-}\left(\left[D_{\nu} U\right]_{-}\left[D_{\beta} G^{\mu \nu}\right]_{+}-\left[D_{\beta} G^{\mu \nu}\right]_{+}\left[D_{\nu} U\right]_{-}\right)\right)$ & $\mathbf{1 2 4}$ \\
$+i B_{24} \operatorname{Tr}\left(\left[D_{\mu} U\right]_{-}\left[D_{\nu} U\right]_{-}\left([\chi]_{+}\left[G^{\mu \nu}\right]_{+}+\left[G^{\mu \nu}\right]_{+}[\chi]_{+}\right)\right)$ & $\overline{179}$ \\
$+i B_{25} \operatorname{Tr}\left(\left[D_{\mu} U\right]_{-}[\chi]_{+}\left[D_{\nu} U\right]_{-}\left[G^{\mu \nu}\right]_{+}\right)$ & $\mathbf{1 8 0}$ \\
$+i B_{26} \operatorname{Tr}\left(\left[D_{\mu} U\right]_{-}\left[D_{\nu} U\right]_{-}\left[G^{\mu \nu}\right]_{+}\right) \operatorname{Tr}\left([\chi]_{+}\right)$ & $\overline{181}$ \\
$+i B_{27} \operatorname{Tr}\left(\left[D_{\mu} U\right]_{-}\left(\left[D_{\nu} \chi\right]_{-}\left[G^{\mu \nu}\right]_{+}-[\chi]_{-}\left[D_{\nu} G^{\mu \nu}\right]_{+}-\left[G^{\mu \nu}\right]_{+}\left[D_{\nu} \chi\right]_{-}+\left[D_{\nu} G^{\mu \nu}\right]_{+}[\chi]_{-}\right)\right)$ & $\mathbf{1 7 3}$ \\
$+i B_{28} \operatorname{Tr}\left(\left[D_{\mu} U\right]_{-}\left(\left[D_{\nu} \chi\right]_{+}\left[H^{\mu \nu}\right]_{+}-[\chi]_{+}\left[D_{\nu} H^{\mu \nu}\right]_{+}+\left[H^{\mu \nu}\right]_{+}\left[D_{\nu} \chi\right]_{+}-\left[D_{\nu} H^{\mu \nu}\right]_{+}[\chi]_{+}\right)\right)$ & $\mathbf{1 7 4}$ \\
$+i B_{29} \operatorname{Tr}\left(\left[D_{\mu} U\right]_{-}\left[H^{\mu \nu}\right]_{+}\right) \operatorname{Tr}\left(\left[D_{\nu} \chi\right]_{+}\right)-\operatorname{Tr}\left(\left[D_{\mu} U\right]_{-}\left[D_{\nu} H^{\mu \nu}\right]_{+}\right) \operatorname{Tr}\left([\chi]_{+}\right)$ & $\mathbf{1 7 5}$ \\
\hline
\end{tabular}

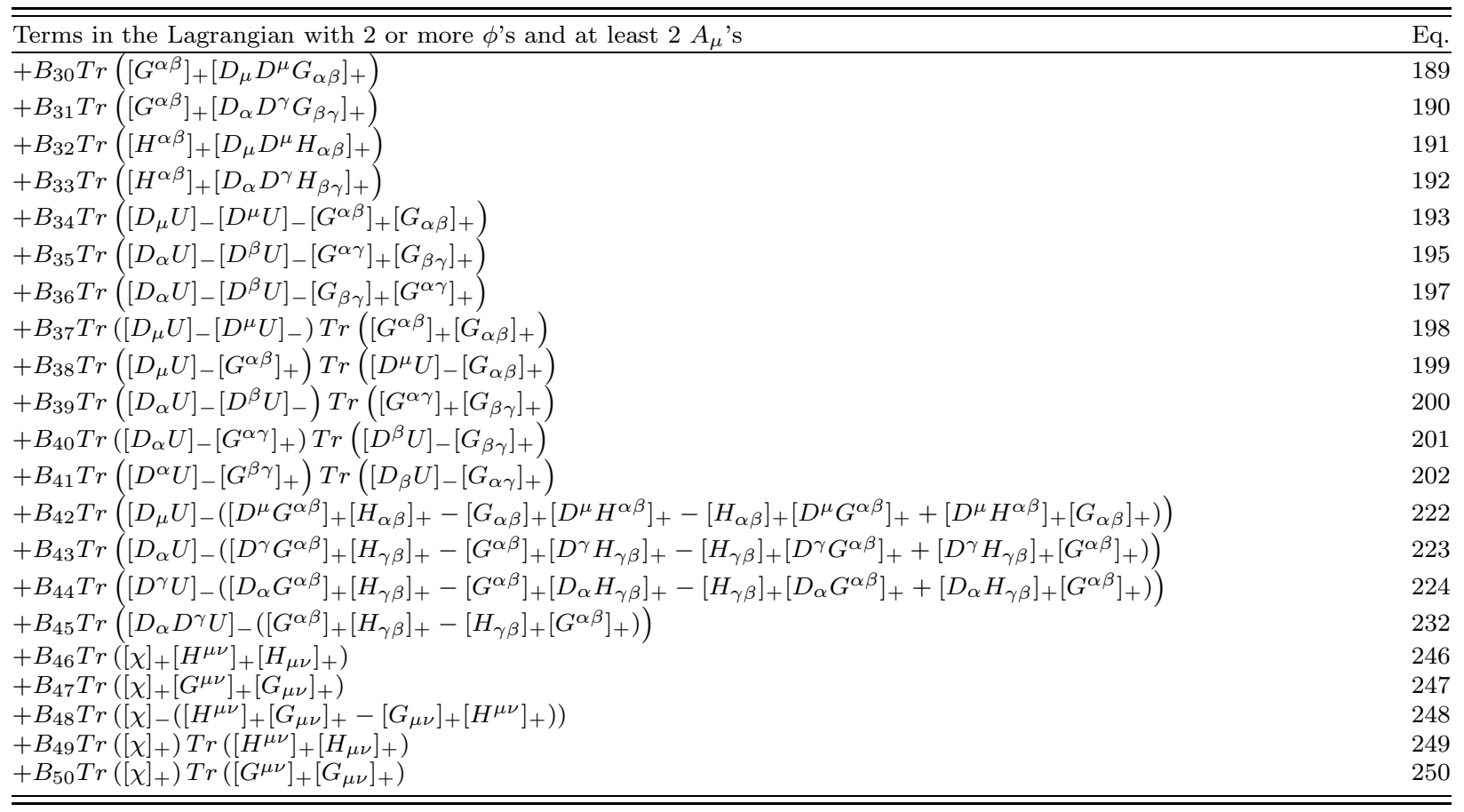

\begin{tabular}{lr}
\hline \hline Terms in the Lagrangian with 2 or more $\phi$ 's and $3 A_{\mu}$ 's & Eq. \\
\hline$+i B_{51} \operatorname{Tr}\left(\left[G^{\mu \nu}\right]_{+}\left[G_{\mu \alpha}\right]_{+}\left[G_{\nu}^{\alpha}\right]_{+}\right)$ & 256 \\
$+i B_{52} \operatorname{Tr}\left(\left[G^{\mu \nu}\right]_{+}\left[H_{\mu \alpha}\right]_{+}\left[H_{\nu}^{\alpha}\right]_{+}\right)$ & \\
\hline \hline
\end{tabular}


TABLE III. Terms in the Lagrangian with 4 or more $\phi$ 's, sorted according to the minimum number of electromagnetic fields $A_{\mu}$. The formulas are general, but the sorting order and number of $\phi$ 's depends on the specific assumptions described in Sec. IV B and the caption of Table III. The double covariant derivative $D_{\mu} D_{\nu} A$ is assumed to be symmetric in its indices in accord with Eq. (27).

\begin{tabular}{|c|c|}
\hline 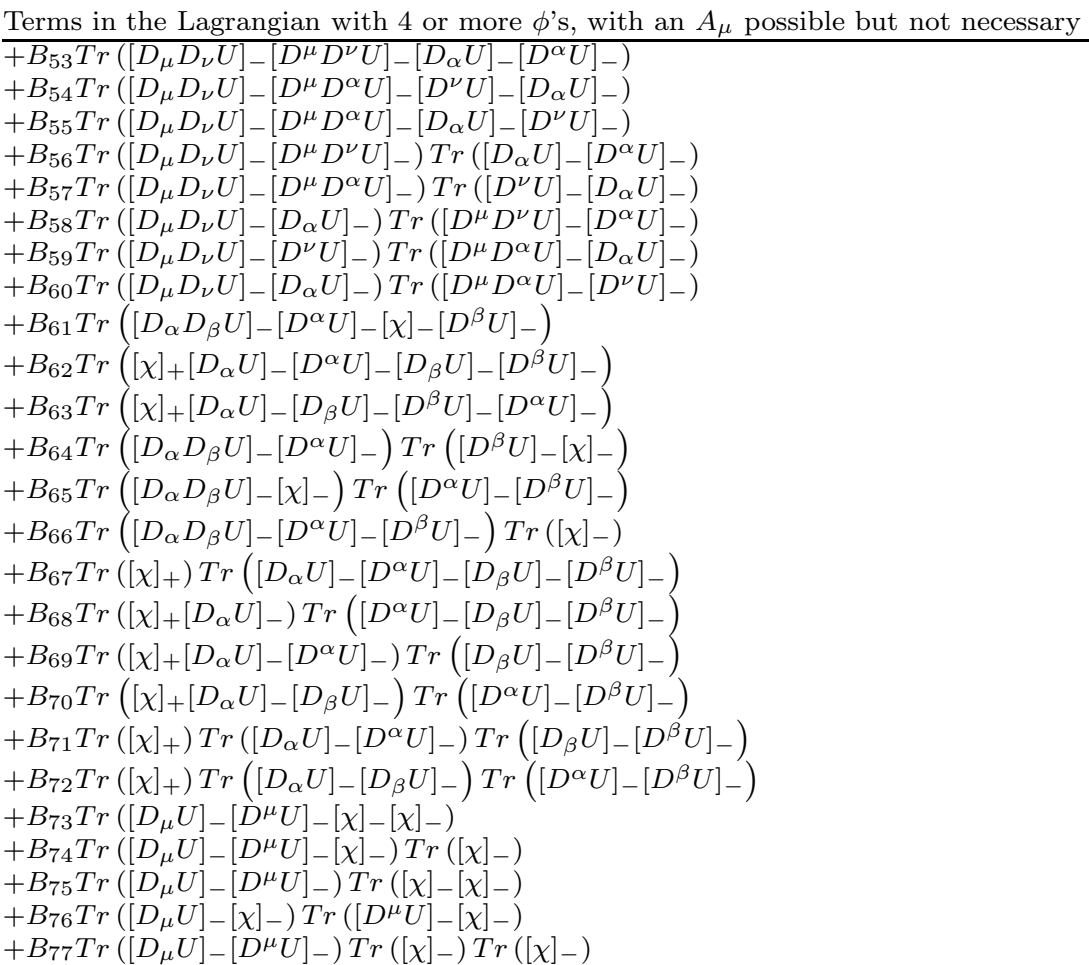 & $\begin{array}{l}\mathrm{Eq} \\
17 \\
18 \\
19 \\
23 \\
64 \\
25 \\
66 \\
67 \\
88 \\
91 \\
93 \\
99 \\
100 \\
101 \\
104 \\
106 \\
107 \\
108 \\
109 \\
110 \\
163 \\
169 \\
168 \\
170 \\
172\end{array}$ \\
\hline
\end{tabular}

\begin{tabular}{lr}
\hline \hline Terms in the Lagrangian with 4 or more $\phi^{\prime}$ s and at least $1 A_{\mu}$ & Eq. \\
\hline$+i B_{78} \operatorname{Tr}\left(\left[D_{\mu} D_{\beta} U\right]_{-}\left(\left[D^{\beta} U\right]_{-}\left[D_{\nu} U\right]_{-}\left[H^{\mu \nu}\right]_{+}+\left[H^{\mu \nu}\right]_{+}\left[D_{\nu} U\right]_{-}\left[D^{\beta} U\right]_{-}\right)\right)$ & 119 \\
$+i B_{79} \operatorname{Tr}\left(\left[D_{\mu} D_{\beta} U\right]_{-}\left(\left[D_{\nu} U\right]_{-}\left[D^{\beta} U\right]_{-}\left[H^{\mu \nu}\right]_{+}+\left[H^{\mu \nu}\right]_{+}\left[D^{\beta} U\right]_{-}\left[D_{\nu} U\right]_{-}\right)\right)_{-}$ & $\mathbf{1 2 0}$ \\
$+i B_{80} \operatorname{Tr}\left(\left[G^{\mu \nu}\right]_{+}\left(\left[D_{\mu} U\right]_{-}\left[D_{\nu} U\right]_{-}\left[D_{\alpha} U\right]_{-}\left[D^{\alpha} U\right]_{-}-\left[D_{\alpha} U\right]_{-}\left[D^{\alpha} U\right]_{-}\left[D_{\nu} U\right]_{-}\left[D_{\mu} U\right]_{-}\right)\right)$ & $\mathbf{1 2 9}$ \\
$+i B_{81} \operatorname{Tr}\left(\left[G^{\mu \nu}\right]_{+}\left(\left[D_{\mu} U\right]_{-}\left[D_{\alpha} U\right]_{-}\left[D_{\nu} U\right]_{-}\left[D^{\alpha} U\right]_{-}-\left[D_{\alpha} U\right]_{-}\left[D_{\nu} U\right]_{-}\left[D^{\alpha} U\right]_{-}\left[D_{\mu} U\right]_{-}\right)\right)$ & $\mathbf{1 3 0}$ \\
$+i B_{82} \operatorname{Tr}\left(\left[G^{\mu \nu}\right]_{+}\left[D_{\mu} U\right]_{-}\left[D_{\alpha} U\right]_{-}\left[D^{\alpha} U\right]_{-}\left[D_{\nu} U\right]_{-}\right)$ & $\mathbf{1 3 1}$ \\
$+i B_{83} \operatorname{Tr}\left(\left[G^{\mu \nu}\right]_{+}\left[D_{\alpha} U\right]_{-}\left[D_{\mu} U\right]_{-}\left[D_{\nu} U\right]_{-}\left[D^{\alpha} U\right]_{-}\right)$ & $\mathbf{1 3 8}$ \\
$+i B_{84} \operatorname{Tr}\left(\left[D_{\mu} D_{\beta} U\right]_{-}\left[D^{\beta} U\right]_{-}\right) \operatorname{Tr}\left(\left[D_{\nu} U\right]_{-}\left[H^{\mu \nu}\right]_{+}\right)$ & $\mathbf{1 3 9}$ \\
$+i B_{85} \operatorname{Tr}\left(\left[D_{\mu} D_{\beta} U\right]_{-}\left[D_{\nu} U\right]_{-}\right) \operatorname{Tr}\left(\left[D^{\beta} U\right]_{-}\left[H^{\mu \nu}\right]_{+}\right)$ \\
$+i B_{86} \operatorname{Tr}\left(\left[D_{\mu} D_{\beta} U\right]_{-}\left[H^{\mu \nu}\right]_{+}\right) \operatorname{Tr}\left(\left[D^{\beta} U\right]_{-}\left[D_{\nu} U\right]_{-}\right)$ \\
$+i B_{87} \operatorname{Tr}\left(\left[G^{\mu \nu}\right]_{+}\left[D_{\alpha} U\right]_{-}\right) \operatorname{Tr}\left(\left[D^{\alpha} U\right]_{-}\left[D_{\mu} U\right]_{-}\left[D_{\nu} U\right]_{-}\right)$ \\
$+i B_{88} \operatorname{Tr}\left(\left[G^{\mu \nu}\right]_{+}\left[D_{\mu} U\right]_{-}\left[D_{\nu} U\right]_{-}\right) \operatorname{Tr}\left(\left[D_{\alpha} U\right]_{-}\left[D^{\alpha} U\right]_{-}\right)$ \\
$+i B_{89} \operatorname{Tr}\left(\left[G^{\mu \nu}\right]_{+}\left(\left[D_{\mu} U\right]_{-}\left[D_{\alpha} U\right]_{-}-\left[D_{\alpha} U\right]_{-}\left[D_{\mu} U\right]_{-}\right)\right) \operatorname{Tr}\left(\left[D^{\alpha} U\right]_{-}\left[D_{\nu} U\right]_{-}\right)$ & $\mathbf{1 4 0}$ \\
$+i B_{90} \operatorname{Tr}\left(\left[D_{\mu} U\right]_{-}\left[D_{\nu} U\right]_{-}\left([\chi]_{-}\left[H^{\mu \nu}\right]_{+}-\left[H^{\mu \nu}\right]_{+}[\chi]_{-}\right)\right)$ & $\mathbf{1 4 2}$ \\
$+i B_{91} \operatorname{Tr}\left(\left[D_{\mu} U\right]_{-}\left[H^{\mu \nu}\right]_{+}\right) \operatorname{Tr}\left(\left[D_{\nu} U\right]_{-}[\chi]_{-}\right)$ & $\mathbf{1 4 3}$ \\
\hline \hline
\end{tabular}




\begin{tabular}{lr}
\hline \hline Terms in the Lagrangian with 4 or more $\phi^{\prime}$ s and at least $2 A_{\mu}^{\prime}$ 's & Eq. \\
\hline$+B_{92} \operatorname{Tr}\left(\left[D_{\mu} U\right]_{-}\left[D^{\mu} U\right]_{-}\left[H^{\alpha \beta}\right]_{+}\left[H_{\alpha \beta}\right]_{+}\right)$ & 203 \\
$+B_{93} \operatorname{Tr}\left(\left[D_{\alpha} U\right]_{-}\left[D^{\beta} U\right]_{-}\left[H^{\alpha \gamma}\right]_{+}\left[H_{\beta \gamma}\right]_{+}\right)$ & 205 \\
$+B_{94} \operatorname{Tr}\left(\left[D_{\alpha} U\right]_{-}\left[D^{\beta} U\right]_{-}\left[H_{\beta \gamma}\right]_{+}\left[H^{\alpha \gamma}\right]_{+}\right)$ & 207 \\
$+B_{95} \operatorname{Tr}\left(\left[D_{\mu} U\right]_{-}\left[D^{\mu} U\right]_{-}\right) \operatorname{Tr}\left(\left[H^{\alpha \beta}\right]_{+}\left[H_{\alpha \beta}\right]_{+}\right)$ & 208 \\
$+B_{96} \operatorname{Tr}\left(\left[D_{\mu} U\right]_{-}\left[H^{\alpha \beta}\right]_{+}\right) \operatorname{Tr}\left(\left[D^{\mu} U\right]_{-}\left[H_{\alpha \beta}\right]_{+}\right)$ & 209 \\
$+B_{97} \operatorname{Tr}\left(\left[D_{\alpha} U\right]_{-}\left[D^{\beta} U\right]_{-}\right) \operatorname{Tr}\left(\left[H^{\alpha \gamma}\right]_{+}\left[H_{\beta \gamma}\right]_{+}\right)$ & 210 \\
$+B_{98} \operatorname{Tr}\left(\left[D_{\alpha} U\right]_{-}\left[H^{\alpha \gamma}\right]_{+}\right) \operatorname{Tr}\left(\left[D^{\beta} U\right]_{-}\left[H_{\beta \gamma}\right]_{+}\right)$ & 211 \\
$+B_{99} \operatorname{Tr}\left(\left[D^{\alpha} U\right]_{-}\left[H^{\beta \gamma}\right]_{+}\right) \operatorname{Tr}\left(\left[D_{\beta} U\right]_{-}\left[H_{\alpha \gamma}\right]_{+}\right)$ & 212 \\
\hline \hline
\end{tabular}


TABLE IV. Terms in the Lagrangian with 6 or more $\phi$ 's. The formulas are general, but the sorting order and number of $\phi$ 's depends on the specific assumptions described in Sec. IVB and the caption of Table II.

Terms in the Lagrangian with 6 or more $\phi$ 's, with an $A_{\mu}$ possible but not necessary

$+B_{100} \operatorname{Tr}\left(\left[D_{\alpha} U\right]_{-}\left[D^{\alpha} U\right]_{-}\left[D_{\beta} U\right]_{-}\left[D^{\beta} U\right]_{-}\left[D_{\gamma} U\right]_{-}\left[D^{\gamma} U\right]_{-}\right)$

$+B_{101} \operatorname{Tr}\left(\left[D_{\alpha} U\right]_{-}\left[D^{\alpha} U\right]_{-}\left[D_{\beta} U\right]_{-}\left[D_{\gamma} U\right]_{-}\left[D^{\beta} U\right]_{-}\left[D^{\gamma} U\right]_{-}\right)$

$+B_{102} \operatorname{Tr}\left(\left[D_{\alpha} U\right]_{-}\left[D^{\alpha} U\right]_{-}\left[D_{\beta} U\right]_{-}\left[D_{\gamma} U\right]_{-}\left[D^{\gamma} U\right]_{-}\left[D^{\beta} U\right]_{-}\right)$

$+B_{103} \operatorname{Tr}\left(\left[D_{\alpha} U\right]_{-}\left[D_{\beta} U\right]_{-}\left[D_{\gamma} U\right]_{-}\left[D^{\alpha} U\right]_{-}\left[D^{\beta} U\right]_{-}\left[D^{\gamma} U\right]_{-}\right)$

$+B_{104} \operatorname{Tr}\left(\left[D_{\mu} U\right]_{-}\left[D^{\mu} U\right]_{-}\right) \operatorname{Tr}\left(\left[D_{\alpha} U\right]_{-}\left[D^{\alpha} U\right]_{-}\left[D_{\beta} U\right]_{-}\left[D^{\beta} U\right]_{-}\right)$

$+B_{105} \operatorname{Tr}\left(\left[D_{\mu} U\right]_{-}\left[D_{\nu} U\right]_{-}\right) \operatorname{Tr}\left(\left[D^{\mu} U\right]_{-}\left[D^{\nu} U\right]_{-}\left[D_{\alpha} U\right]_{-}\left[D^{\alpha} U\right]_{-}\right)$

$+B_{106} \operatorname{Tr}\left(\left[D_{\mu} U\right]_{-}\left[D^{\mu} U\right]_{-}\left[D_{\alpha} U\right]_{-}\right) \operatorname{Tr}\left(\left[D_{\beta} U\right]_{-}\left[D^{\beta} U\right]_{-}\left[D^{\alpha} U\right]_{-}\right)$

$+B_{107} \operatorname{Tr}\left(\left[D_{\mu} U\right]_{-}\left[D_{\nu} U\right]_{-}\left[D_{\alpha} U\right]_{-}\right) \operatorname{Tr}\left(\left[D^{\mu} U\right]_{-}\left[D^{\nu} U\right]_{-}\left[D^{\alpha} U\right]_{-}\right)$

$+B_{108} \operatorname{Tr}\left(\left[D_{\mu} U\right]_{-}\left[D_{\nu} U\right]_{-}\left[D_{\alpha} U\right]_{-}\right) \operatorname{Tr}\left(\left[D^{\mu} U\right]_{-}\left[D^{\alpha} U\right]_{-}\left[D^{\nu} U\right]_{-}\right)$

$+B_{109} \operatorname{Tr}\left(\left[D_{\mu} U\right]_{-}\left[D^{\mu} U\right]_{-}\right) \operatorname{Tr}\left(\left[D_{\alpha} U\right]_{-}\left[D^{\alpha} U\right]_{-}\right) \operatorname{Tr}\left(\left[D_{\beta} U\right]_{-}\left[D^{\beta} U\right]_{-}\right)$

$+B_{110} \operatorname{Tr}\left(\left[D_{\mu} U\right]_{-}\left[D^{\mu} U\right]_{-}\right) \operatorname{Tr}\left(\left[D_{\alpha} U\right]_{-}\left[D_{\beta} U\right]_{-}\right) \operatorname{Tr}\left(\left[D^{\alpha} U\right]_{-}\left[D^{\beta} U\right]_{-}\right)$

$+B_{111} \operatorname{Tr}\left(\left[D_{\mu} U\right]_{-}\left[D_{\nu} U\right]_{-}\right) \operatorname{Tr}\left(\left[D^{\mu} U\right]_{-}\left[D^{\alpha} U\right]_{-}\right) \operatorname{Tr}\left(\left[D^{\nu} U\right]_{-}\left[D_{\alpha} U\right]_{-}\right)$

TABLE V. Terms in the Lagrangian with 1 or more $\phi$ 's and an $\epsilon_{\alpha \beta \gamma \delta},\left(\epsilon_{0123}=+1\right)$, sorted according to the minimum number of electromagnetic fields $A_{\mu}$. The formulas are general, but the sorting order and number of $\phi$ 's depends on the specific assumptions described in Sec. IVB and the caption of Table II.

\begin{tabular}{|c|c|}
\hline Terms in the Lagrangian with 1 or more $\phi$ 's, an $\epsilon_{\alpha \beta \gamma \delta}$, and at least $1 A_{\mu}$ & Eq. \\
\hline$\overline{+A_{1} \operatorname{Tr}\left(\left[D^{\mu} U\right]_{-}\left(\left[D^{\nu} \chi\right]_{+}\left[G^{\gamma \delta}\right]_{+}-[\chi]_{+}\left[D^{\nu} G^{\gamma \delta}\right]_{+}-\left[G^{\gamma \delta}\right]_{+}\left[D^{\nu} \chi\right]_{+}+\left[D^{\nu} G^{\gamma \delta}\right]_{+}[\chi]_{+}\right)\right) \epsilon_{\mu \nu \gamma \delta}}$ & 176 \\
\hline Terms in the Lagrangian with 1 or more $\phi$ 's, an $\epsilon_{\alpha \beta \gamma \delta}$, and at least $2 A_{\mu}$ 's & $\mathrm{F}$ \\
\hline$\overline{+i A_{2} \operatorname{Tr}\left(\left[D^{\mu} U\right]_{-}\left(\left[D^{\nu} G^{\alpha \beta}\right]_{+}\left[G_{\alpha}^{\gamma}\right]_{+}-\left[G^{\alpha \beta}\right]_{+}\left[D^{\nu} G_{\alpha}^{\gamma}\right]_{+}\right)\right) \epsilon_{\mu \nu \beta \gamma}}$ & 228 \\
\hline$+i A_{3} \operatorname{Tr}\left(\left[D^{\mu} U\right]_{-}\left(\left[D_{\alpha} G^{\alpha \beta}\right]_{+}\left[G^{\gamma \delta}\right]_{+}-\left[G^{\alpha \beta}\right]_{+}\left[D_{\alpha} G^{\gamma \delta}\right]_{+}-\left[D_{\alpha} G^{\gamma \delta}\right]_{+}\left[G^{\alpha \beta}\right]_{+}+\left[G^{\gamma \delta}\right]_{+}\left[D_{\alpha} G^{\alpha \beta}\right]_{+}\right)\right) \epsilon_{\mu \beta \gamma \delta}$ & 229 \\
\hline$+i A_{4} \operatorname{Tr}\left([\chi]_{-}\left[G^{\alpha \beta}\right]_{+}\left[G^{\gamma \delta}\right]_{+}\right) \epsilon_{\alpha \beta \gamma \delta}$ & 252 \\
\hline$+i A_{5} \operatorname{Tr}\left([\chi]_{+}\left(\left[H^{\alpha \beta}\right]_{+}\left[G^{\gamma \delta}\right]_{+}-\left[G^{\gamma \delta}\right]_{+}\left[H^{\alpha \beta}\right]_{+}\right)\right) \epsilon_{\alpha \beta \gamma \delta}$ & 250 \\
\hline$+i A_{6} \operatorname{Tr}\left([\chi]_{-}\right) \operatorname{Tr}\left(\left[G^{\alpha \beta}\right]_{+}\left[G^{\gamma \delta}\right]_{+}\right) \epsilon_{\alpha \beta \gamma \delta}$ & 255 \\
\hline
\end{tabular}


TABLE VI. Terms in the Lagrangian with 3 or more $\phi$ 's and an $\epsilon_{\alpha \beta \gamma \delta},\left(\epsilon_{0123}=+1\right)$, sorted according to the minimum number of electromagnetic fields $A_{\mu}$. The formulas are general, but the sorting order and number of $\phi$ 's depends on the specific assumptions described in Sec. IVB and the caption of Table $\llbracket$. The double covariant derivative $D_{\mu} D_{\nu} A$ is assumed to be symmetric in its indices in accord with Eq. (27).

\begin{tabular}{lr}
\hline \hline Terms in the Lagrangian with 3 or more $\phi^{\prime}$ s, an $\epsilon_{\alpha \beta \gamma \delta}$, and at least $1 A_{\mu}$ & Eq. \\
\hline$+A_{7} \operatorname{Tr}\left(\left[D^{\alpha} D^{\beta} U\right]_{-}\left(\left[D_{\beta} U\right]_{-}\left[D^{\gamma} U\right]_{-}\left[G^{\mu \nu}\right]_{+}-\left[G^{\mu \nu}\right]_{+}\left[D^{\gamma} U\right]_{-}\left[D_{\beta} U\right]_{-}\right)\right) \epsilon_{\alpha \gamma \mu \nu}$ & 113 \\
$+A_{8} \operatorname{Tr}\left(\left[D^{\alpha} D^{\beta} U\right]_{-}\left(\left[D^{\gamma} U\right]_{-}\left[D_{\beta} U\right]_{-}\left[G^{\mu \nu}\right]_{+}-\left[G^{\mu \nu}\right]_{+}\left[D_{\beta} U\right]_{-}\left[D^{\gamma} U\right]_{-}\right)\right) \epsilon_{\alpha \gamma \mu \nu}$ & $15]$ \\
$+A_{9} \operatorname{Tr}\left(\left[D^{\alpha} D_{\beta} U\right]_{-}\left[D^{\gamma} U\right]_{-}\left[G^{\beta \nu}\right]_{+}\left[D^{\delta} U\right]_{-}\right) \epsilon_{\alpha \gamma \delta \nu}$ & 117 \\
$+A_{10} \operatorname{Tr}\left(\left[D^{\alpha} D^{\beta}\right]_{-}\left(\left[D_{\beta} U\right]_{-}\left[D^{\gamma} H^{\mu \nu}\right]_{+}+\left[D^{\gamma} H^{\mu \nu}\right]_{+}\left[D_{\beta} U\right]_{-}\right)\right) \epsilon_{\alpha \gamma \mu \nu}$ & 126 \\
$+A_{11} \operatorname{Tr}\left(\left[D^{\alpha} D^{\beta}\right]_{-}\left(\left[D^{\gamma} U\right]_{-}\left[D_{\beta} H^{\mu \nu}\right]_{+}+\left[D_{\beta} H^{\mu \nu}\right]_{+}\left[D^{\gamma} U\right]_{-}\right)\right) \epsilon_{\alpha \gamma \mu \nu}$ & $184]$ \\
$+A_{12} \operatorname{Tr}\left(\left[D^{\mu} U\right]_{-}\left[D^{\nu} U\right]_{-}\left([\chi]_{-}\left[G^{\gamma \delta}\right]_{+}+\left[G^{\gamma \delta}\right]_{+}[\chi]_{-}\right)\right) \epsilon_{\mu \nu \gamma \delta}$ & 185 \\
$+A_{13} \operatorname{Tr}\left(\left[D^{\mu} U\right]_{-}[\chi]_{-}\left[D^{\nu} U\right]_{-}\left[G^{\gamma \delta}\right]_{+}\right) \epsilon_{\mu \nu \gamma \delta}$ & 187 \\
$+A_{14} \operatorname{Tr}\left(\left[D^{\mu} U\right]_{-}\left[D^{\nu} U\right]_{-}\left[G^{\gamma \delta}\right]_{+}\right) \operatorname{Tr}\left([\chi]_{-}\right) \epsilon_{\mu \nu \gamma \delta}$ & $\mathbf{1 8 8}$ \\
$+A_{15} \operatorname{Tr}\left(\left[D^{\mu} U\right]_{-}\left[D^{\nu} U\right]_{-}\left([\chi]_{+}\left[H^{\gamma \delta}\right]_{+}-\left[H^{\gamma \delta}\right]_{+}[\chi]_{+}\right)\right) \epsilon_{\mu \nu \gamma \delta}$ & $\mathbf{1 7 7}$ \\
$+A_{16} \operatorname{Tr}\left(\left[D^{\mu} U\right]_{-}\left[H^{\gamma \delta}\right]_{+}\right) \operatorname{Tr}\left(\left[D^{\nu} U\right]_{-}[\chi]_{+}\right) \epsilon_{\mu \nu \gamma \delta}$ & $\mathbf{1 7 8}$ \\
$+A_{17} \operatorname{Tr}\left(\left[D^{\mu} U\right]_{-}\left(\left[D^{\nu} \chi\right]_{-}\left[H^{\gamma \delta}\right]_{+}-[\chi]_{-}\left[D^{\nu} H^{\gamma \delta}\right]_{+}+\left[H^{\gamma \delta}\right]_{+}\left[D^{\nu} \chi\right]_{-}-\left[D^{\nu} H^{\gamma \delta}\right]_{+}[\chi]_{-}\right)\right) \epsilon_{\mu \nu \gamma \delta}$ & \\
$+A_{18}\left(\operatorname{Tr}\left(\left[D^{\mu} U\right]_{-}\left[H^{\gamma \delta}\right]_{+}\right) \operatorname{Tr}\left(\left[D^{\nu} \chi\right]_{-}\right)-\operatorname{Tr}\left(\left[D^{\mu} U\right]_{-}\left[D^{\nu} H^{\gamma \delta}\right]_{+}\right) \operatorname{Tr}\left([\chi]_{-}\right)\right) \epsilon_{\mu \nu \gamma \delta}$ & \\
\hline \hline
\end{tabular}

\begin{tabular}{lr}
\hline \hline Terms in the Lagrangian with 3 or more $\phi$ 's, an $\epsilon_{\alpha \beta \gamma \delta}$, and at least $2 A_{\mu}$ 's & Eq. \\
\hline$+i A_{19} \operatorname{Tr}\left(\left[D_{\mu} U\right]_{-}\left[D^{\mu} U\right]_{-}\left(\left[G^{\alpha \beta}\right]_{+}\left[H^{\gamma \delta}\right]_{+}-\left[H^{\gamma \delta}\right]_{+}\left[G^{\alpha \beta}\right]_{+}\right)\right) \epsilon_{\alpha \beta \gamma \delta}$ & 213 \\
$+i A_{20} \operatorname{Tr}\left(\left[D^{\mu} U\right]_{-}\left[D^{\nu} U\right]_{-}\left(\left[G^{\alpha \beta}\right]_{+}\left[H_{\alpha}^{\gamma}\right]_{+}+\left[H_{\alpha}^{\gamma}\right]_{+}\left[G^{\alpha \beta}\right]_{+}\right)\right) \epsilon_{\mu \nu \beta \gamma}$ & 214 \\
$+i A_{21} \operatorname{Tr}\left(\left[D^{\mu} U\right]_{-}\left[G^{\alpha \beta}\right]_{+}\left[D^{\nu} U\right]_{-}\left[H_{\alpha}^{\gamma}\right]_{+}\right) \epsilon_{\mu \nu \beta \gamma}$ & 215 \\
$+i A_{22} \operatorname{Tr}\left(\left[D^{\mu} U\right]_{-}\left(\left[D_{\alpha} U\right]_{-}\left[G^{\alpha \beta}\right]_{+}\left[H^{\gamma \delta}\right]_{+}-\left[H^{\gamma \delta}\right]_{+}\left[G^{\alpha \beta}\right]_{+}\left[D_{\alpha} U\right]_{-}\right)\right) \epsilon_{\mu \beta \gamma \delta}$ & 216 \\
$+i A_{23} \operatorname{Tr}\left(\left[D^{\mu} U\right]_{-}\left(\left[D^{\nu} H^{\alpha \beta}\right]_{+}\left[H_{\alpha}^{\gamma}\right]_{+}-\left[H^{\alpha \beta}\right]_{+}\left[D^{\nu} H_{\alpha}^{\gamma}\right]_{+}\right)\right) \epsilon_{\mu \nu \beta \gamma}$ & 225 \\
$+i A_{24} \operatorname{Tr}\left(\left[D^{\mu} U\right]_{-}\left(\left[D_{\alpha} H^{\alpha \beta}\right]_{+}\left[H^{\gamma \delta}\right]_{+}-\left[H^{\alpha \beta}\right]_{+}\left[D_{\alpha} H^{\gamma \delta}\right]_{+}-\left[D_{\alpha} H^{\gamma \delta}\right]_{+}\left[H^{\alpha \beta}\right]_{+}+\left[H^{\gamma \delta}\right]_{+}\left[D_{\alpha} H^{\alpha \beta}\right]_{+}\right)\right) \epsilon_{\mu \beta \gamma \delta}$ & 226 \\
$+i A_{25} \operatorname{Tr}\left([\chi]_{-}\left[H^{\alpha \beta}\right]_{+}\left[H^{\gamma \delta}\right]_{+}\right) \epsilon_{\alpha \beta \gamma \delta}$ & 251 \\
$+i A_{26} \operatorname{Tr}\left([\chi]_{-}\right) \operatorname{Tr}\left(\left[H^{\alpha \beta}\right]_{+}\left[H^{\gamma \delta}\right]_{+}\right) \epsilon_{\alpha \beta \gamma \delta}$ & 254 \\
\hline \hline
\end{tabular}

TABLE VII. Terms in the Lagrangian with 5 or more $\phi$ 's and an $\epsilon_{\alpha \beta \gamma \delta},\left(\epsilon_{0123}=+1\right)$, sorted according to the minimum number of electromagnetic fields $A_{\mu}$. The formulas are general, but the sorting order and number of $\phi$ 's depends on the specific assumptions described in Sec. IVB and the caption of Table $\llbracket$. The double covariant derivative $D_{\mu} D_{\nu} A$ is assumed to be symmetric in its indices in accord with Eq. (27).

\begin{tabular}{lr}
\hline \hline Terms in the Lagrangian with 5 or more $\phi$ 's and an $\epsilon_{\alpha \beta \gamma \delta}$, with an $A_{\mu}$ possible but not necessary & Eq. \\
\hline$+i A_{27} \operatorname{Tr}\left(\left[D^{\mu} D^{\nu} U\right]_{-}\left(\left[D_{\nu} U\right]_{-}\left[D^{\beta} U\right]_{-}\left[D^{\gamma} U\right]_{-}\left[D^{\delta} U\right]_{-}+\left[D^{\delta} U\right]_{-}\left[D^{\gamma} U\right]_{-}\left[D^{\beta} U\right]_{-}\left[D_{\nu} U\right]_{-}\right)\right) \epsilon_{\mu \beta \gamma \delta}$ & 52 \\
$+i A_{28} \operatorname{Tr}\left([\chi]_{-}\left[D_{\alpha} U\right]_{-}\left[D_{\beta} U\right]_{-}\left[D_{\gamma} U\right]_{-}\left[D_{\delta} U\right]_{-}\right) \epsilon^{\alpha \beta \gamma \delta}$ & \\
\hline \hline & 94 \\
\hline \hline Terms in the Lagrangian with 5 or more $\phi^{\prime}$ s, an $\epsilon_{\alpha \beta \gamma \delta}$, and at least $1 A_{\mu}$ & Eq. \\
\hline$+A_{29} \operatorname{Tr}\left(\left[H^{\mu \nu}\right]_{+}\left(\left[D^{\alpha} U\right]_{-}\left[D^{\beta} U\right]_{-}\left[D_{\gamma} U\right]_{-}\left[D^{\gamma} U\right]_{-}+\left[D_{\gamma} U\right]_{-}\left[D^{\gamma} U\right]_{-}\left[D^{\beta} U\right]_{-}\left[D^{\alpha} U\right]_{-}\right)\right) \epsilon_{\mu \nu \alpha \beta}$ & 133 \\
$+A_{30} \operatorname{Tr}\left(\left[H^{\mu \nu}\right]_{+}\left(\left[D^{\alpha} U\right]_{-}\left[D_{\gamma} U\right]_{-}\left[D^{\beta} U\right]_{-}\left[D^{\gamma} U\right]_{-}+\left[D_{\gamma} U\right]_{-}\left[D^{\beta} U\right]_{-}\left[D^{\gamma} U\right]_{-}\left[D^{\alpha} U\right]_{-}\right)\right) \epsilon_{\mu \nu \alpha \beta}$ & $134]$ \\
$+A_{31} \operatorname{Tr}\left(\left[H^{\mu \nu}\right]_{+}\left[D^{\alpha} U\right]_{-}\right) \operatorname{Tr}\left(\left[D^{\beta} U\right]_{-}\left[D_{\gamma} U\right]_{-}\left[D^{\gamma} U\right]_{-}\right) \epsilon_{\mu \nu \alpha \beta}$ & 144 \\
$+A_{32} \operatorname{Tr}\left(\left[H^{\mu \nu}\right]_{+}\left(\left[D^{\alpha} U\right]_{-}\left[D^{\gamma} U\right]_{-}+\left[D^{\gamma} U\right]_{-}\left[D^{\alpha} U\right]_{-}\right)\right) \operatorname{Tr}\left(\left[D^{\beta} U\right]_{-}\left[D_{\gamma} U\right]_{-}\right) \epsilon_{\mu \nu \alpha \beta}$ & 145 \\
\hline \hline
\end{tabular}


TABLE VIII. Terms in the Lagrangian proportional to the classical equation of motion. The lowest order equation of motion operator $\mathcal{O}_{E O M}^{(2)}$ is given by Eq. (260). The double covariant derivative $D_{\mu} D_{\nu} A$ is assumed to be symmetric in its indices in accord with Eq. (27), as are all multiple covariant derivatives.

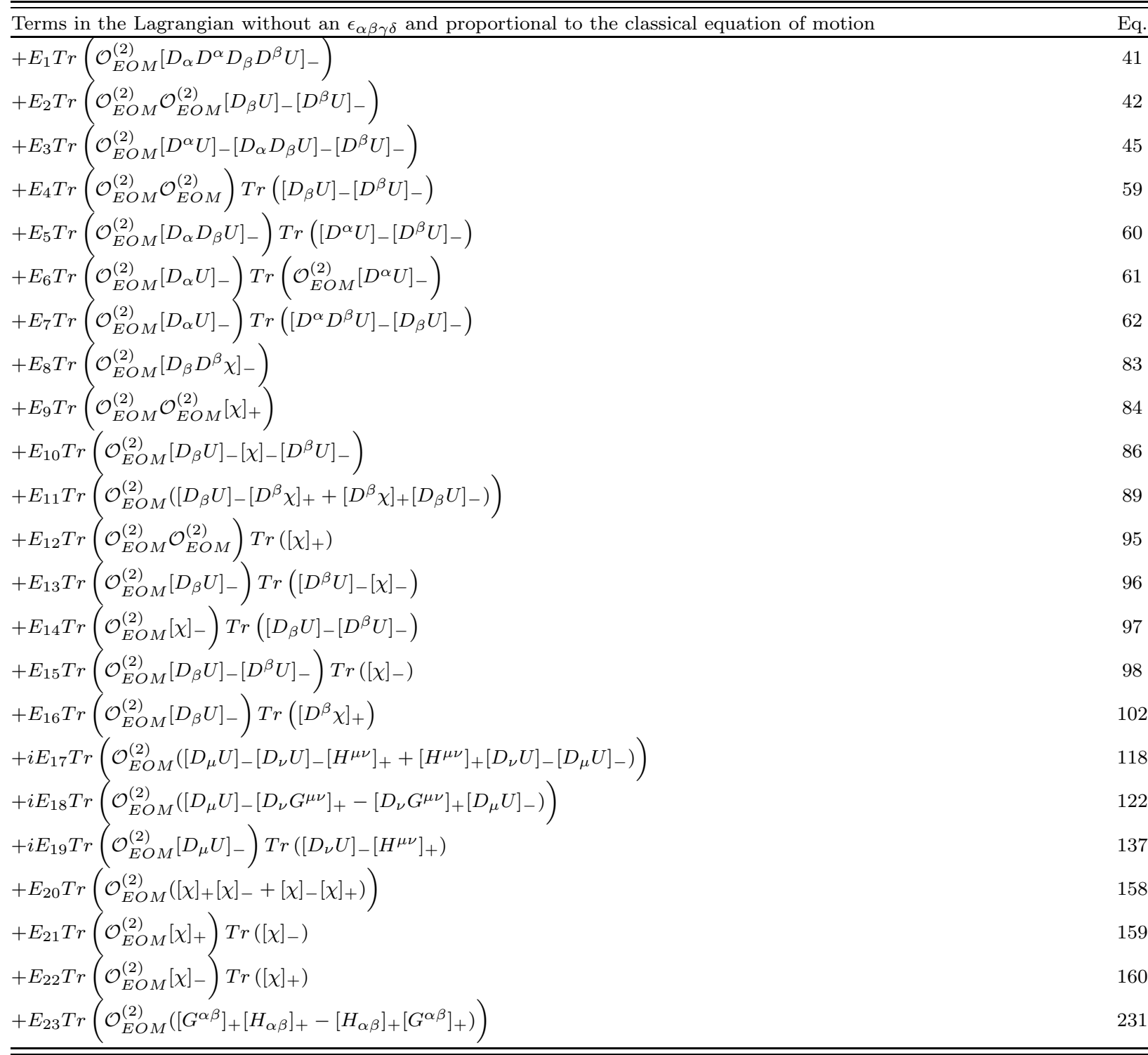

\begin{tabular}{lr}
\hline \hline Terms in the Lagrangian with an $\epsilon_{\alpha \beta \gamma \delta},\left(\epsilon_{0123}=+1\right)$, and proportional to the classical equation of motion & Eq. \\
\hline$+i E_{24} \operatorname{Tr}\left(\mathcal{O}_{E O M}^{(2)}\left[D^{\alpha} U\right]_{-}\left[D^{\beta} U\right]_{-}\left[D^{\gamma} U\right]_{-}\left[D^{\delta} U\right]_{-}\right) \epsilon_{\alpha \beta \gamma \delta}$ & 16 \\
$+E_{25} \operatorname{Tr}\left(\mathcal{O}_{E O M}^{(2)}\left(\left[D^{\beta} U\right]_{-}\left[D^{\gamma} U\right]_{-}\left[G^{\mu \nu}\right]_{+}-\left[G^{\mu \nu}\right]_{+}\left[D^{\gamma} U\right]_{-}\left[D^{\beta} U\right]_{-}\right)\right) \epsilon_{\beta \gamma \mu \nu}$ & 111 \\
$+E_{26} \operatorname{Tr}\left(\mathcal{O}_{E O M}^{(2)}\left[D^{\beta} U\right]_{-}\left[G^{\mu \nu}\right]_{+}\left[D^{\gamma} U\right]_{-}\right) \epsilon_{\beta \gamma \mu \nu}$ & 112 \\
$+E_{27} \operatorname{Tr}\left(\mathcal{O}_{E O M}^{(2)}\left(\left[D^{\beta} U\right]_{-}\left[D^{\gamma} H^{\mu \nu}\right]_{+}+\left[D^{\gamma} H^{\mu \nu}\right]_{+}\left[D^{\beta} U\right]_{-}\right)\right) \epsilon_{\beta \gamma \mu \nu}$ & 125 \\
$+i E_{28} \operatorname{Tr}\left(\mathcal{O}_{E O M}^{(2)}\left[H^{\alpha \beta}\right]_{+}\left[H^{\gamma \delta}\right]_{+}\right) \epsilon_{\alpha \beta \gamma \delta}$ & 233 \\
$+i E_{29} \operatorname{Tr}\left(\mathcal{O}_{E O M}^{(2)}\left[G^{\alpha \beta}\right]_{+}\left[G^{\gamma \delta}\right]_{+}\right) \epsilon_{\alpha \beta \gamma \delta}$ & 235 \\
\hline \hline
\end{tabular}


TABLE IX. Application of trace relations: The first column contains the relevant equation numbers of structures which are related by trace relations. The second column refers to the specific trace relation which has been applied. The third column denotes the equation number of the structure which we have chosen to eliminate.

\begin{tabular}{|c|c|c|}
\hline related structures & trace relation & structure eliminated \\
\hline$(42),(44),(59),(61)$ & A13) & $(44)$ \\
\hline$(43),(45),(60), 62)$ & (A9) & (43) \\
\hline$(\sqrt{47}),(50), 63), 65)$ & A13) & (50) \\
\hline$(48),(49),(51),(64),(66),(67)$ & (A9) & (51) \\
\hline$(54)-(58),(68)-(74)$ & A19) & $(58)$ \\
\hline$(68),(\sqrt[69]{69}),(75),(76)$ & A13) & (69) \\
\hline$(\sqrt{70}),(\sqrt{71}),(76),(77)$ & (A11) & (71) \\
\hline$(85),(86),(96),(97),(98)$ & $(\overline{\mathrm{A} 10})$ & (85) \\
\hline (87), (88), (99),(100),(101) & A8 & (87) \\
\hline (91)-(93),(106)-(108) & (A16) & (92) \\
\hline$(\sqrt{104}),(105),(109),(\sqrt{110})$ & (A13) & (105) \\
\hline$(119)-(121),(138)-(140)$ & $(\overline{\mathrm{A} 9}$ & (121) \\
\hline (161),(162), (165), (167), (169),(171) & A12) & (162) \\
\hline$(163),(164),(166),(168),(170),(172)$ & A12) & (164) \\
\hline$(193),(194),(198),(199)$ & (A13) & (194) \\
\hline$(195)-(197),(200)-(202)$ & (A9) & (196) \\
\hline$(203),(204),(208),(209)$ & (A13) & (204) \\
\hline$(205)-(207),(210)-212$ & (A9) & (206) \\
\hline
\end{tabular}

TABLE X. Application of epsilon relations: The first column contains the relevant equation numbers of structures which are related by epsilon relations. The second column denotes the equation number of the structure which we have chosen to eliminate.

\begin{tabular}{|c|c|}
\hline related structures & structure eliminated \\
\hline$(46),(52),(53)$ & $(53)$ \\
\hline$(112),(114),(117)$ & (114) \\
\hline (111),(113),(115),(116) & (116) \\
\hline$(\sqrt{125}),(\sqrt{126}),(\sqrt{127}),(\sqrt{128})$ & $(\overline{128})$ \\
\hline$(133),(134),(135),(136)$ & $(135),(136)$ \\
\hline$(215),(217),(220)$ & $(\overline{217}),(220)$ \\
\hline$(213),(214),(216),(218),(219),(221)$ & $(218),(219),(221)$ \\
\hline$(225),(226),(227)$ & (227) \\
\hline$(228), 229,(230)$ & (230) \\
\hline$(233),(234)$ & (234) \\
\hline$(235),(236)$ & $(236)$ \\
\hline (258) & (258) \\
\hline
\end{tabular}

